\title{
Motifs Génériques.
}

\author{
FrédéRIC DÉGLISe (*)
}

RÉSUMÉ - Cet article a pour but de montrer que tout motif mixte triangulé au sens de V. Voevodsky définit canoniquement un module de cycles au sens de M. Rost. Notre méthode consiste à interpréter géométriquement les axiomes des modules de cycles dans une catégorie de pro-motifs, dits “motifs génériques". Elle est suffisamment générale pour montrer que toute théorie cohomologique qui induit une réalisation des motifs mixtes triangulés définit canoniquement un module de cycles, ce qui s'applique notamment à la cohomologie de De Rham ou à la cohomologie rigide.

ABstract - The aim of the article is to show that every mixed triangulated motive in the sense of $\mathrm{V}$. Voevodsky determines a canonical cycle module in the sense of M. Rost. Our method consists of interpreting geometrically the axioms of cycle modules in a category of pro-motives called "generic motives". It is general enough to show at the same time that every cohomological theory which induces a realisation of triangulated mixed motives defines a canonical cycle module. This is in particular applicable to De Rham and rigid cohomology.

\section{Remerciements.}

Cet article est extrait de la thèse de l'auteur, réalisée sous la direction de F. Morel. C'est à lui que vont principalement mes remerciements, pour m'avoir transmis ses idées et son savoir mathématique auxquels ce travail doit beaucoup. Je remercie aussi D.-C. Cisinski et J. Wildeshaus pour m'avoir soutenu et aidé pendant la préparation de cet article.

(*) Indirizzo dell'A.: Institut Galilée, Université Paris 13, 99 avenue JeanBaptiste Clément, 93430 Villetaneuse France.

E-mail: deglise@math.univ-paris13.fr 


\section{Notations}

On fixe un corps parfait $k$. Les schémas que l'on considère sans plus de précision sont supposés munis d'une structure de $k$-schéma séparé de type fini. On dit simplement qu'un schéma est lisse lorsqu'il est lisse sur $k$, et on note $\mathscr{L}_{k}$ la catégorie des schémas lisses.

Nous appellerons corps de fonctions tout corps $E$ qui est une extension de type fini de $k$. Nous noterons $\mathscr{E}_{k}$ la catégorie des corps de fonctions. Si $E$ est un corps de fonctions, nous appellerons valuation géométrique toute valuation discrète de $E$ nulle sur $k$ dont l'anneau de valuation est une $k$ algèbre essentiellement de type fini (cf section 3.2 et exemple 3.2.3). Si $X$ est un schéma intègre, nous noterons $\kappa(X)$ son corps de fonctions.

- V. Voevodsky a défini dans [Voe00b] la catégorie triangulée des motifs mixtes géométriques sur $k$, notée $D M_{g m}(k)$. D’une manière générale, nous adoptons les notations de loc. cit. sauf en ce qui concerne les points suivants:

- Le symbole $\mathscr{B}_{k}^{\text {cor }}$ désigne la catégorie additive des schémas lisses munis des correspondances finies.

- Le symbole $\mathscr{N}_{k}^{\mathrm{tr}}$ (resp. $\mathrm{H} \mathscr{N}_{k}^{\mathrm{tr}}$ ) désigne la catégorie abélienne des faisceaux Nisnevich avec transferts (resp. et invariant par homotopie), que nous appelons simplement faisceaux avec transferts (resp. faisceaux homotopiques).

- Pour $X$ un schéma lisse, $\mathrm{L}[X]$ désigne le faisceau avec transferts représenté par $X$.

- Pour les complexes de faisceaux avec transferts, nous adoptons la convention cohomologique. Ainsi, pour un faisceau avec tranferts $F, \underline{\mathrm{C}}^{*}(F)$ désigne le complexe des co-chaînes singulières associé à $F$, obtenu après réindexation à partir du complexe des chaînes singulières associé à $F$ (cf. [Voe00b, 3.2]).

- Pour un morphisme $f: X \rightarrow Y$ de schémas lisses, nous notons simplement $f_{*}$ pour les morphismes $M_{g m}(X) \rightarrow M_{g m}(Y)$ et $M(X) \rightarrow M(Y)$ induits par $f$.

- Pour un complexe motivique $\mathcal{M}$, et pour un entier naturel $n$, nous posons:

$$
\begin{aligned}
& \mathcal{M}(n)=\mathbb{Z}(n) \otimes \mathcal{M} \\
& \mathcal{M}\{n\}=\mathbb{Z}(n)[n] \otimes \mathcal{M} \\
& \mathcal{M}((n))=\mathbb{Z}(n)[2 n] \otimes \mathcal{M} \text {. }
\end{aligned}
$$

Nous utiliserons aussi les définitions et notations de la théorie des 
modules de cycles définie par M. Rost dans [Ros96]. Le lecteur trouvera un rappel de cet théorie dans la section 4 .

\section{Introduction}

Cet article est le troisième d'une série de quatre (voir [Dég07] et [Dég06] pour les deux premiers) qui vise à établir une équivalence de catégories entre les modules de cycles (cf. [Ros96]) et une variante non effective des faisceaux homotopiques (i.e. faisceaux invariants par homotopie avec transferts suivant [Voe00b]). Nous nous occupons ici de montrer comment associer à un complexe motivique un module de cycles. Nous donnons notamment une interprétation géométrique des axiomes D1 à D4 des pré-modules de cycles dans la catégorie triangulée des motifs mixtes, en vérifiant en outre les relations R1a à R3e de la théorie de Rost. Ceci nous a mené à étudier finement la fonctorialité du triangle de Gysin défini par Voevodsky, la remarque fondamentale étant que les résidus des modules de cycles sont induits par les morphismes bords de ce triangle.

La théorie de Rost fait intervenir des corps de fonctions. C'est pourquoi l'interprétation mentionnée ci-dessus nous amène à introduire les motifs génériques. Pour un corps de fonction $E$, le motif générique de $E$, noté $M_{g m}(E)$, est le pro-objet formé par tous les schémas lisses dont le corps des fonctions est égal à $E$ - un tel schéma lisse sera appelé un modèle de $E$ dans ce qui suit (cf. définition 3.3.1). La classe des modèles de $E$ contient un ensemble final remarquable formé par les sous- $k$-algèbres de type fini $A$ de $E$ telle que $\operatorname{Spec}(A)$ est lisse. On le note $\mathcal{M}^{l i s}(E / k)$. Notons que les proobjets du type $M_{g m}(E)$ ont aussi été considérés par A. Beilinson dans [Bě̃02] pour caractériser la t-structure motivique sur $D M_{g m}^{e f f}(k)$. Toutefois, nous sommes conduits à considérer non seulement les motifs génériques définis ci-dessus mais aussi leur twist par une puissance de Z(1)[1] quelconque.

Une autre manière d'interpréter ces motifs génériques, qui a été un guide tout au long de ce travail, est de les considérer comme des foncteurs fibres. En effet, à la base de la théorie de Voevodsky se trouve la notion de faisceaux Nisnevich avec transferts invariants par homotopie. Or on peut définir l'évaluation d'un tel faisceau $F$ sur un corps de fonction $E$ : si $X$ est un modèle de $E$, cette évaluation n'est autre que la fibre du faisceau $F$ au point générique du site Nisnevich de $X$. Plus généralement, l'évaluation de $F$ en un motif générique $M_{g m}(E)\{n\}$ pour $n \geq 0$ définit un foncteur exact qui commute aux sommes directes quelconques (cf. 3.4.6), autrement dit un 
foncteur fibre. Notons par ailleurs que ces foncteurs fibres forment une famille conservative. Les morphismes de motifs génériques correspondant aux données $\mathrm{D} 1$ à $\mathrm{D} 4$ des modules de cycles s'interprètent donc comme des foncteurs de spécialisation (i.e. des transformations naturelles de foncteurs fibres).

Une fois ce travail accompli et la théorie des motifs génériques en place, nous donnons la conséquence principale de cette théorie constituée par le théorème 6.2.1:

THÉoRÈme. Soit $\varphi: D M_{g m}(k)^{o p} \rightarrow$ Ab un foncteur additif.

Pour tout $(i, r) \in Z^{2}$ et tout corps de fonctions $E$, on pose:

$$
K_{i}^{\varphi, r}(E)=\lim _{A \in \mathcal{M}^{l i s}(E)} \varphi(M(\operatorname{Spec}(A)\{-i\})(-r)) .
$$

Alors, pour tout $r \in Z, K_{*}^{\varphi, r}$ admet une structure canonique de module de cycles (voir définitions 4.1.1 et 4.2.2).

Dans le cas où $\varphi$ est le foncteur induit par un faisceau homotopique $F$, nous poserons encore $\hat{F}_{*}=K_{*}^{\varphi, 0}$. Ceci définit un foncteur des faisceaux homotopiques vers les modules de cycles. Dans le dernier article de la série annoncée en début d'introduction, nous montrerons que ce foncteur est pleinement fidèle et qu'il induit une équivalence de catégories entre la catégorie stable des faisceaux homotopiques et la catégorie des modules de cycles (cf. [Dég03]).

Par ailleurs, si une théorie cohomologique $H^{*}: \mathscr{L}_{k}^{o p} \rightarrow \mathscr{b b}$ s'étend en un foncteur $R_{H}: D M_{g m}(k)^{o p} \rightarrow \not b b$ de telle sorte que $H^{i}(X)=$ $=R_{H}\left(M_{g m}(X)[-i]\right)$, elle induit d'après le théorème ci-dessus un module de cycles. Une telle extension a été construite par A. Huber pour la cohomologie de De Rham, ce qui constitue déjà un exemple non trivial. De plus, dans un travail en préparation, on construira de telles réalisations et ceci pour la plupart des théories cohomologiques en géométrie algébrique. La cohomologie rigide est notre motivation première. L'existence d'une structure de module de cycles sur la cohomologie rigide des corps de fonctions est entièrement nouvelle dans ce domaine et ne résulte pas de calculs élémentaires.

Dans chaque cas, le théorème ci-dessus s'applique et relie le module de cycles ainsi obtenu avec la suite spectrale de coniveau (cf. partie II, section $6.3)$.

Terminons cette introduction par les développements indépendants du théorème principal de [Dég03] auxquels mène cet article. 
L'étude du triangle de Gysin est complétée dans [Dég05] qui s'appuie abondamment sur les résultats obtenus ici. Dans cette pré-publication, nous établissons par ailleurs un isomorphisme canonique de complexes entre le complexe de Gersten associé à la réalisation d'une théorie cohomologique au sens précédent, et le complexe à coefficients dans le module de cycles associé (complexe de Cousin).

Soulignons enfin que le travail d'interprétation géométrique des axiomes de Rost effectué dans la première partie nous permettra de construire un analogue de la suite spectrale de Serre de la topologie algébrique, associée dans notre cas à un morphisme lisse et une théorie cohomologique qui induit une réalisation. Cette suite spectrale constitue également une extension au cas de la cohomologie motivique de la suite spectrale définie par Rost pour les modules de cycles (cf. [Ros96]).

PLAN DÉTAILLÉ. L’article est naturellement divisé en deux parties.

La première partie peut-être lue indépendamment de la deuxième. Elle est constituée d'une étude de la fonctorialité des motifs mixtes triangulés qui sera exploitée dans la deuxième partie.

La première section montre comment les correspondances finies permettent de définir un transfert sur les motifs mixtes géométriques pour un morphisme fini équidimensionnel de schémas lisses qui n'est autre que sa transposée. Nous prouvons une formule de projection qui fait intervenir les transferts mais aussi une formule de compatibilité avec la fonctorialité naturelle des motifs dans un cas non transverse (cf. proposition 1.1.4).

La deuxième section est constituée par l'étude du triangle de Gysin déjà défini dans [Voe00b]. Cette étude vient raffiner la note [Dég04] avec notamment une compatibilité entre le triangle de Gysin et la transposée d'un morphisme fini équidimensionnel (cf. proposition 2.5.2). Par ailleurs, contrairement à cette note, nous n'admettons pas le fait que l'isomorphisme entre cohomologie motivique de bidegré $(2 n, n)$ avec le groupe de Chow des cycles de codimension $n$ est compatible avec le pullback et le pushout. Nous donnons une preuve élémentaire de ces deux propriétés pour la cohomologie motivique de poids 1 dans 2.2.4 et 2.2.6. Notons que la compatibilité de cet isomorphisme avec le pushtout est un des points clés de 2.5.2. La fin de cette section montre une compatibilité facile entre le triangle de Gysin et le produit tensoriel (cf. 2.6.1) qui toutefois cache le signe de la formule R3e (cf. remarque 2.6.2 pour plus de précisions). La formule de la proposition 2.6.5 illustre clairement ce que l'on entend par une interprétation géo- 
métrique des axiomes des modules de cycles au vu de sa contrepartie dans la deuxième partie (formule R3d du lemme 5.4.8).

La deuxième partie exploite la première partie dans le but de démontrer le théorème central de l'article (cf. théorème 6.2.1).

Dans la troisième section, nous introduisons la catégorie des motifs génériques (cf. définition 3.3.1) notée $D M_{g m}^{(0)}(k)$. Notre définition s'appuie sur la notion de pro-objets pour laquelle nous faisons quelques rappels au début de cette section. La fin de cette section est consacrée à l'interprétation des motifs génériques en tant que "points" ou plus précisément foncteurs fibres pour les faisceaux homotopiques. Cette interprétation fait intervenir naturellement la t-structure homotopique sur la catégorie des complexes motiviques. Ici se trouve le point de départ du théorème 6.2.1. D'un point de vue conceptuel, si $F$ est un faisceau homotopique, le module de cycles $\hat{F}$ est la restriction du faisceau $F$ à une catégorie conservative de points, la catégorie des motifs génériques.

La quatrième section contient quelques rappels sur les modules de cycles ainsi que l'introduction de la catégorie $\tilde{\mathscr{E}}_{k}$ (cf. 5.1) qui décrit les morphismes structuraux des modules de cycles par générateurs et relations. La cinquième section constitue le coeur technique du théorème 6.2.1. Nous construisons un foncteur $M^{(0)}: \tilde{\mathscr{E}}_{k} \rightarrow D M_{g m}^{(0)}(k)$ (cf. théorème 5.1.1) qui joue le rôle de "module de cycles universel". Le reste de cette section se concentre sur cette construction qui s'appuie sur la première partie de l'article. La méthode consiste à construire chacun des morphismes de $\tilde{\mathscr{E}}_{k}$ sur des modèles des corps de fonctions en jeu, à montrer que la construction se localise aux points génériques des modèles et à montrer l'indépendance de la définition par rapport aux modèles choisis. Les relations des morphismes de $\tilde{\mathscr{E}}_{k}$ se déduisent directement des résultats de la première partie.

La sixième section permet d'appliquer les résultats obtenus. On notera qu'on déduit immédiatement de 5.1.1 le fait que $K_{*}^{\varphi, r}$ est un pré-module de cycles. Il nous faut utiliser un résultat supplémentaire pour obtenir le fait que c'est un module de cycles et l'argument nécessite l'hypothèse que l'on travaille sur un corps parfait. Ce résultat nécessite le théorème (2.3) de [Ros96] et le calcul du motif générique d'une extension transcendante pure d'un corps de fonction (cf. corollaire 6.1.3). La fin de cette section permet de relier les motifs génériques et le module de cycles $K_{*}^{\varphi, r}$ avec les suites spectrales obtenues à partir de la filtration par coniveau. Ce lien est particulièrement éclairé et approfondi dans [Dég05]. Enfin, nous citons en remarque une réduction facile de la conjecture de Beilinson-Soulé qui exploite le fait que la cohomologie motivique est un module de cycles. 


\section{Morphismes dans la catégorie des motifs}

\section{Transferts}

Pour alléger les notations, on pose $X Y=X \times_{k} Y$ pour tous schémas $X$ et $Y$.

\section{1 - Transposée dans les correspondances finies}

Soient $X$ et $Y$ deux schémas lisses. Rappelons qu'une correspondance finie de $X$ vers $Y$ est un cycle de $X Y$ dont le support est fini équidimensionnel sur $X$.

Si $f$ est un morphisme de schémas de $X$ vers $Y$, le cycle associé à son graphe $\Gamma_{f}$ définit une correspondance finie de $X$ vers $Y$ que l'on désignera encore par $f$. Notons que si $f$ est fini équidimensionnel, $\Gamma_{f}$ est fini équidimensionnel sur $Y$. Ceci nous permet d'adopter la définition suivante:

DÉFInition 1.1.1. Soient $X$ et $Y$ deux schémas lisses et $f: X \rightarrow Y$ un morphisme fini équidimensionnel.

On appelle transposée de $f$, notée ${ }^{t} f$, la correspondance finie de $Y$ vers $X$ dont le cycle est associé au graphe de $f$ vu comme sous-schéma fermé de $Y X$ via l'isomorphisme de permutation des facteurs.

On note aussi $f^{*}: M(Y) \rightarrow M(X)$ le morphisme de $D M_{-}^{\text {eff }}(k)$ qui s'en déduit.

La transposée vérifie les propriétés élémentaires suivantes dont on laisse la démonstration au lecteur:

LEMme 1.1.2. Soit $f: Y \rightarrow X$ un morphisme fini équidimensionnel dans $\mathscr{L}_{k}$. Alors, on a les relations suivantes:

(1) Si $\alpha \in \mathrm{c}(Y)$,$Z est une correspondance finie, \alpha \circ^{t} f=\left(f \times 1_{Z}\right)_{*}(\alpha)$.

(2) Si $g: Z \rightarrow Y$ est un morphisme fini équidimensionnel, ${ }^{t}(f \circ g)=$ $={ }^{t} g \circ{ }^{t} f$.

On termine cette section par une formule de projection généralisée. Pour la formuler, on introduit la définition suivante:

DÉfinition 1.1.3. Soit $Y^{\prime} \stackrel{q}{\rightarrow} Y$ un carré de morphismes de schémas.

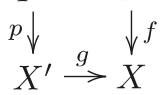


Nous dirons qu'il est quasi-cartésien si il est commutatif et cartésien sur les espaces topologiques sous-jacents.

Considérons un carré quasi-cartésien comme ci-dessus tel que $X, X^{\prime}, Y$ et $Y^{\prime}$ sont lisses, $f$ est fini équidimensionnel et $g$ est dominant. Alors $R=\kappa(Y) \otimes_{\kappa(X)} \kappa\left(X^{\prime}\right)$ est l'anneau total des fractions de $Y^{\prime}$. Ainsi, $\operatorname{Spec}(R)$ est en bijection avec l'ensemble des composantes connexes de $Y^{\prime}$. Pour tout $x \in \operatorname{Spec}(R)$, on note $Y^{\prime}(x)$ l'adhérence de $x$ dans $Y^{\prime}$ vu comme un sousschéma réduit et on pose $q_{x}=\left.q\right|_{Y^{\prime}(x)}, p_{x}=\left.q\right|_{Y^{\prime}(x)}$.

Proposition 1.1.4. Considérons les hypothèses et notations qui précèdent. Alors la formule suivante est satisfaite:

$$
{ }^{t} f \circ g=\sum_{x \in \operatorname{Spec}(R)} \lg _{R}\left(R_{x}\right) \cdot q_{x} \circ{ }^{t} p_{x}
$$

Preuve: Soit $\Gamma_{f}$ (resp. $\Gamma_{g}$ ) le graphe $\operatorname{de} f$ (resp. $g$ ).

Considérons l'intersection schématique $W$ de $\Gamma_{g} Y$ et $X^{\prime} \Gamma_{f}$ dans $X^{\prime} X Y$. Le schéma $W$ est isomorphe à $X^{\prime} \times_{X} Y$ et son image dans $X^{\prime} Y$ est égale à l'image du morphisme $X^{\prime} \times_{X} Y \rightarrow X^{\prime} Y$. Par ailleurs, chaque composante de cette intersection est propre et l'ensemble de ces componsantes est en bijection avec $\operatorname{Spec}(R)$. Par hypothèse, $W_{\text {red }}=Y^{\prime}$ est lisse. Il en résulte que pour tout $x \in \operatorname{Spec}(R)$, vu comme un point générique de $W,\left(\mathcal{O}_{W, x}\right)_{\text {red }}$ est un anneau local de Cohen-Macaulay - i.e. de profondeur égale à sa dimension ; cf. [Ful98], A.7. Donc l'anneau $\mathcal{O}_{W, x}$ est de Cohen-Macaulay. Dès lors, par application de la proposition 7.1 de [Ful98], la multiplicité d'intersection de $x$ dans $W$ est égale à la longueur de l'anneau artinien $\mathcal{O}_{W, x}$.

Ainsi, la correspondance finie ${ }^{t} f \circ g$ est le cycle associé au sous-schéma $W$ dans $X^{\prime} Y$ :

$$
{ }^{t} f \circ g=\sum_{x \in \operatorname{Spec}(R)} \lg \left(\mathcal{O}_{W, x}\right) \cdot\left[Y^{\prime}(x)\right]_{X^{\prime} Y} .
$$

Comme on l'a déjà dit, $R$ est isomorphe à l'anneau total des fractions de $W$. Donc pour tout $x \in \operatorname{Spec}(R), \lg \left(\mathcal{O}_{W, x}\right)=\lg \left(R_{x}\right)$. Enfin, un calcul immédiat montre que pour $x \in \operatorname{Spec}(R), q_{x} \circ{ }^{t} p_{x}=\left[Y^{\prime}(x)\right]_{Y^{\prime} Y}$.

\section{2 - Cup-produit externe}

Pour un schéma lisse $X$, on note $\Delta_{X}: X \rightarrow X \times_{k} X$ l'immersion diagonale associée. 
DÉfinition 1.2.1. Soient $\mathcal{M}$ et $\mathcal{N}$ des objets de $D M_{-}^{\text {eff }}(k)$ et $\alpha: M(X) \rightarrow$ $\rightarrow \mathcal{M}, \beta: M(X) \rightarrow \mathcal{N}$ des morphismes de $D M_{-}^{\text {eff }}(k)$.

On appelle cup-produit externe de $\alpha$ et $\beta$ au-dessus de $X$ la composée de morphismes

$$
M(X) \stackrel{\Delta_{X}}{\longrightarrow} M(X) \otimes M(X) \stackrel{\alpha \otimes \beta}{\longrightarrow} \mathcal{M} \otimes \mathcal{N} .
$$

On le note $\alpha \bigotimes_{X} \beta$ ou simplement $\alpha \bowtie \beta$.

Exemple 1.2.2. Considérons $\alpha \in \mathrm{c}(X)$,$Y et \beta \in \mathrm{c}(X)$,$Z . Un calcul$ élémentaire montre alors: $M(\alpha) \bigotimes M(\beta)=M\left(p_{X Y Z}^{X Y}{ }^{*}(\alpha) \cdot p_{X Y Z}^{X Z}{ }^{*}(\beta)\right)$.

1.2.3. On considèrera particulièrement ce cup-produit dans le cas où, étant donnés deux entiers naturels $n$ et $m, \mathcal{M}=Z(m)[2 m]$ et $\mathcal{N}=Z(n)[2 n]$.

Or $Z(*)[2 *]$ est muni d'une structure d'anneau canonique pour le produit tensoriel de $D M_{-}^{\text {eff }}(k)$. En effet, pour tous entiers naturels $n$ et $m$, on dispose d'un isomorphisme canonique $\varepsilon: Z(m)[2 m] \otimes Z(n)[2 n] \rightarrow$ $\rightarrow Z(n+m)[2(m+n)]$. Si $\alpha: M(X) \rightarrow Z(m)[2 m]$ et $\beta: M(X) \rightarrow Z(n)[2 n]$ sont deux morphismes, on définit le cup-produit interne associé à $\varepsilon$ de $\alpha$ et $\beta$ comme le morphisme

$$
\alpha \smile \beta=\varepsilon \circ(\alpha \bigotimes \beta) .
$$

Ce morphisme correspond à une classe de cohomologie motivique qui n'est autre que le cup-produit de $\alpha$ et $\beta$.

Les formules suivantes résultent facilement de la théorie classique d'intersection des cycles et nous laissons leur démonstration au lecteur:

Proposition 1.2.4. Soit $f: Y \rightarrow X$ un morphisme de schémas lisses. Considérons $\alpha: M(X) \rightarrow \mathcal{M}, \quad \beta: M(X) \rightarrow \mathcal{N}, \quad \gamma: M(X) \rightarrow \mathcal{P}$ des morphismes de $D M_{-}^{\text {eff }}(k)$. Alors, les relations suivantes sont vérifiées:

(1) $(\alpha \rrbracket \beta) \bigotimes \gamma=\alpha \rrbracket(\beta \bigotimes \gamma)$.

(2) $\left(\alpha \bigotimes_{X} \beta\right) \circ f_{*}=\left(\alpha \circ f_{*}\right) \bigotimes_{Y}\left(\beta \circ f_{*}\right)$.

Si de plus $f$ est fini équidimensionnel,

(3) $\left(f_{*} \bigotimes_{Y} 1_{M(Y)}\right) \circ f^{*}=1_{M(X)} \bigotimes_{X} f^{*}$.

(4) $\left(1_{M(Y)} \bigotimes_{Y} f_{*}\right) \circ f^{*}=f^{*} \bigotimes_{X} 1_{M(X)}$. 


\section{Triangle de Gysin}

\section{1 - Motifs relatifs}

DÉfinition 2.1.1. Soient $X$ un schéma lisse et $Y$ un sous-schéma lisse de $X$.

On note $\mathrm{L}[X / Y]$ le conoyau dans la catégorie $\mathscr{N}_{k}^{\mathrm{tr}} \mathrm{du}$ morphisme induit par l'inclusion canonique $\mathrm{L}[Y] \rightarrow \mathrm{L}[X]$. On note aussi $M\left(X / Y=\underline{\mathrm{C}}^{*} \mathrm{~L}[X / Y)\right]$ le complexe motivique correspondant.

On appelle paire fermée tout couple $(X, Z)$ formé d'un schéma lisse $X$ et d'un sous-schéma fermé $Z$ de $X$. Nous dirons simplement que $(X, Z)$ est lisse si $Z$ est lisse et que $(X, Z)$ est de codimension $n$ si $Z$ est de codimension pure $n$ dans $X$.

$\mathrm{Si}(X, Z)$ est une paire fermée, on désigne encore le motif $M(X / X-Z)$ par la notation $M_{Z}(X)$ (suivant Voevodsky) ou encore $M(X, Z)$ suivant la clarté de l'écriture. On l'appelle le motif relatif associé à la paire fermée $(X, Z)$. Ce motif s'inscrit dans le triangle distingué canonique suivant:

$$
M(X-Z) \stackrel{j_{*}}{\longrightarrow} M(X) \rightarrow M_{Z}(X) \stackrel{+1}{\longrightarrow} .
$$

Un morphisme de paires fermées $(f, g):(Y, T) \rightarrow(X, Z)$ est un carré commutatif de morphismes de schémas

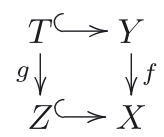

qui est quasi-cartésien (cf. définition 1.1.3). On dit que ce morphisme est cartésien si le carré ci-dessus est cartésien dans la catégorie des schémas. Si $\mathcal{P}$ est une propriété des morphismes de schémas, on dira que $(f, g)$ vérifie $\mathcal{P}$ si $f$ vérifie $\mathcal{P}$. Un tel morphisme induit un morphisme $(f, g)_{*}: M_{T}(Y) \rightarrow M_{Z}(X)$ dans $D M_{-}^{e f f}(k)$.

2.1.2. Soit $(f, g):(Y, T) \rightarrow(X, Z)$ un morphisme de paires fermées fini et équidimensionnel. Par définition, le carré suivant

$$
\begin{array}{rl}
Y-T C & Y \\
h \downarrow & \downarrow f \\
X-Z \hookrightarrow & X
\end{array}
$$

est cartésien. Donc, d'après le corollaire 1.1.3, on en déduit un unique morphisme induit, dessiné en pointillé dans le diagramme suivant de la 
catégorie des faisceaux avec transferts:

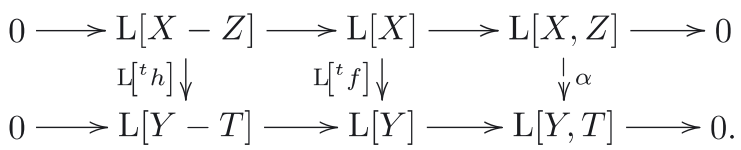

DÉfinition 2.1.3. Avec les notations qui précèdent, on pose $(f, g)^{*}=\underline{\mathrm{C}}^{*}(\alpha)$ qui définit donc un morphisme $M_{Z}(X) \rightarrow M_{T}(Y)$.

Par définition, ce morphisme s'inscrit dans le morphisme de triangle distingué suivant:

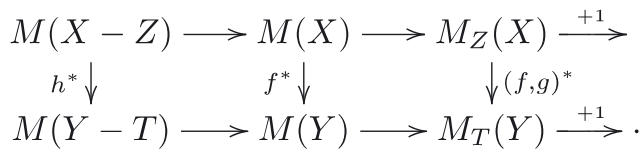

On déduit facilement de la proposition 1.1.4 la formule suivante:

Proposition 2.1.4. Considérons un carré commutatif

$$
\begin{gathered}
\left(Y^{\prime}, T^{\prime}\right) \stackrel{\left(p^{\prime}, q^{\prime}\right)}{\longrightarrow}(Y, T) \\
\left(f^{\prime}, g^{\prime}\right) \downarrow \\
\quad\left(X^{\prime}, Z^{\prime}\right) \stackrel{(p, q)}{\longrightarrow}(X, Z)
\end{gathered}
$$

de paires fermées tel que $(f, g)$ est fini équidimensionnel et $(p, q)$ dominant. On suppose que $X, X^{\prime}$ et $Y$ sont connexes et que le carré induit sur $X$, $X^{\prime}, Y, Y^{\prime}$ est quasi-cartésien.

Soit $R=\kappa\left(X^{\prime}\right) \otimes_{\kappa(X)} \kappa(Y)$. Pour tout $x \in \operatorname{Spec}(R)$, on note $\left(f_{x}^{\prime}, g_{x}^{\prime}\right)$ et $\left(p_{x}^{\prime}, q_{x}^{\prime}\right)$ les restrictions respectives de $\left(f^{\prime}, g^{\prime}\right)$ et $\left(p^{\prime}, q^{\prime}\right)$ correspondant à la composante connexe de $Y^{\prime}$ de point générique $x$.

Alors,

$$
(f, g)^{*}(p, q)_{*}=\sum_{x \in \operatorname{Spec}(R)} \lg _{R}\left(R_{x}\right) \cdot\left(p_{x}^{\prime}, q_{x}^{\prime}\right)_{*}\left(f_{x}^{\prime}, g_{x}^{\prime}\right)^{*}
$$

\section{2 - Théorème de pureté}

Soit $(X, Z)$ une paire fermée lisse. On considère l'espace de déformation au cône normal $D_{Z} X=B_{Z}\left(\mathrm{~A}_{X}^{1}\right)-B_{Z} X$ où $B_{Z}\left(\mathrm{~A}_{X}^{1}\right)$ (resp. $B_{Z} X$ ) désigne l'éclatement de $A_{X}^{1}$ en $0 \times Z$ (resp. $X$ en $\left.Z\right), B_{Z} X$ étant vu comme un sousschéma fermé de $B_{Z}\left(\mathrm{~A}_{X}^{1}\right)$ par l'immersion évidente. Alors $D_{Z} X$ est plat sur 
$A^{1}$ et sa fibre en 1 (resp. 0) est isomorphe à $X$ (resp. au fibré normal $N_{Z} X$ de $Z$ dans $X$ ). Notons pour finir que $D_{Z} Z=\mathrm{A}_{Z}^{1}$ est un sous-schéma fermé de $D_{Z} X$. On en déduit donc des morphismes de paires fermées

$$
(X, Z) \stackrel{d}{\longrightarrow}\left(D_{Z} X, \mathrm{~A}_{Z}^{1}\right) \stackrel{d^{\prime}}{\longleftarrow}\left(N_{Z} X, Z\right) .
$$

Il résulte de [Dég04, prop. 2.4] que les morphismes induits

$$
M_{Z}(X) \stackrel{d_{*}}{\longrightarrow} M\left(D_{Z} X, \mathrm{~A}_{Z}^{1}\right) \stackrel{d_{*}^{\prime \prime}}{\longleftarrow} M_{Z}\left(N_{Z} X\right)
$$

sont des isomorphismes. Notons que les morphismes $d$ et $d^{\prime}$ sont naturels par rapport aux morphismes cartésiens de paires fermées.

\subsection{1 - Motif de Thom}

DÉfinition 2.2.1. Soient $X$ un schéma lisse, et $E / X$ un fibré vectoriel.

On considère la paire fermée $(E, X)$ où $X$ est vu comme un sous-schéma fermé à travers la section nulle. On pose $M \operatorname{Th}(E / X=M(E, X))$, et on appelle ce motif relatif le motif de Thom de $E / X$.

On notera souvent abusivement $M \operatorname{Th}(E)=M \operatorname{Th}(E / X)$.

Comme les motifs relatifs, le motif de Thom est fonctoriel par rapport aux morphismes de paires fermées $(f, g):(F, Y) \rightarrow(E, X)$ où $E / X$ et $F / Y$ sont des fibrés vectoriels. On notera en particulier que $f$ peut très bien ne pas être un morphisme de fibrés vectoriels. Toutefois, lorsqu'on s'est donné un monomorphisme $\phi: E \rightarrow F$ de fibrés vectoriels sur $X$, on notera simplement $M \mathrm{Th}(\phi)$ le morphisme sur les espaces de Thom associés.

Notons par ailleurs que le motif de Thom est contravariant par rapport aux morphismes finis équidimensionnels de paires fermées (cf. 2.1.2).

2.2.2. Soient $E / X($ resp. $F / Y)$ un fibré vectoriel et $\mathcal{E}($ resp. $\mathcal{F})$ le dual de son faisceau des sections. On note $E \boxplus F$ le fibré vectoriel - appelé somme directe extérieure de $E$ et $F-\operatorname{sur} X \times_{k} Y$ associé à l' $\mathcal{O}_{X Y}$-module localement libre $\left(p_{X Y}^{X}\right)^{*} \mathcal{E} \oplus\left(p_{X Y}^{Y}\right)^{*} \mathcal{F}$. Alors,

$$
M \operatorname{Th}(E \boxplus F) \simeq M \operatorname{Th}(E) \otimes M \operatorname{Th}(F .)
$$

Considérons le morphisme de paires fermées $\Delta:(E, X) \rightarrow$ $\rightarrow\left(E \boxplus E, X \times_{k} X\right)$ donné par les morphismes diagonaux évidents. Nous associons à tous morphismes $\alpha: M \operatorname{Th}(E) \rightarrow \mathcal{M}$ et $\beta: M \operatorname{Th}(E) \rightarrow \mathcal{N}$ un 
cup-produit externe:

$$
\alpha \bigotimes_{E / X} \beta=(\alpha \otimes \beta) \circ \Delta_{*} .
$$

On déduit facilement de la proposition 1.2.4 les propriétés analogues suivantes:

Lemme 2.2.3. Soient $X, Y$ des schémas et $E / X, F / Y$ des fibrés vectoriels. On considère un morphisme $(f, g):(F, Y) \rightarrow(E, X)$ et les morphismes suivants dans $D M_{-}^{\text {eff }}(k): \alpha: M \operatorname{Th}(E) \rightarrow \mathcal{M}, \beta: M \operatorname{Th}(E) \rightarrow \mathcal{N}$ et $\gamma: M \operatorname{Th}(E) \rightarrow \mathcal{P}$.

Alors,

(1) $(\alpha \rrbracket \beta) \bigotimes \gamma=\alpha \rrbracket(\beta \bigotimes \gamma)$.

(2) $(\alpha \bigotimes \beta) \circ(f, g)_{*}=\left(\alpha \circ(f, g)_{*}\right) \bigotimes\left(\beta \circ(f, g)_{*}\right)$.

Si de plus $f$ est fini équidimensionnel,

(3) $\left((f, g)_{*} \bigotimes 1_{M T h(F)}\right) \circ(f, g)^{*}=1_{M T h(E)} \bigotimes(f, g)^{*}$.

(4) $\left(1_{M \operatorname{Th}(F)} \bigotimes(f, g)_{*}\right) \circ(f, g)^{*}=(f, g)^{*} \bigotimes 1_{M T h(E)}$.

\subsection{2 - Motif de Tate et transferts}

Soit $X$ un schéma lisse. Si $p: X \rightarrow \operatorname{Spec}(k)$ désigne le morphisme structural de $X$, on note $\tilde{\mathrm{L}}[X]$ le complexe de faisceaux avec transferts Cone $\left(p_{*}\right)[-1]$. Suivant Voevodsky, on pose aussi $\tilde{M}(X)=\underline{\mathrm{C}}^{*}(\tilde{\mathrm{L}}[X])$, appelé motif réduit de $X$, qui s'inscrit dans le triangle distingué

$$
\tilde{M}(X) \rightarrow M(X) \rightarrow Z \rightarrow \tilde{M}(X[1] .)
$$

Si $X$ admet un point rationnel $x$, le triangle ci-dessus est scindé et on obtient un isomorphisme canonique $\tilde{M}(X) \simeq M(X /\{x\})$ avec la notation de 2.1.1.

Suivant [Voe00b], on pose $Z(1)=\tilde{M}\left(\mathbb{P}_{k}^{1}[-2]\right)$. Ce motif est appelé le motif de Tate.

Dans ce qui suit, nous reprenons le calcul du motif de Tate dû à Suslin et Voevodsky afin de déterminer l'action de la transposée d'un morphisme fini équidimensionnel sur sa cohomologie.

Proposition 2.2.4. Le complexe motivique Z(1) est concentré en degré 1 et $\mathrm{H}^{1}(\mathrm{Z}(1)) \simeq \mathrm{G}_{m}$, en tant que faisceau sur $\mathscr{L}_{k}$.

Soit $\varepsilon_{X}: \mathrm{H}^{1}(X ; Z(1)) \rightarrow \mathcal{O}_{X}^{\times}(X)$ l'isomorphisme naturel qui s'en déduit pour un schéma lisse $X$. 
Soient $X$ et $Y$ deux schémas lisses connexes de corps des fonctions respectifs $E$ et $L$ et $f: Y \rightarrow X$ un morphisme fini surjectif. Notons $N_{L / E}$ la norme de $L / E$. Alors, le diagramme suivant est commutatif:

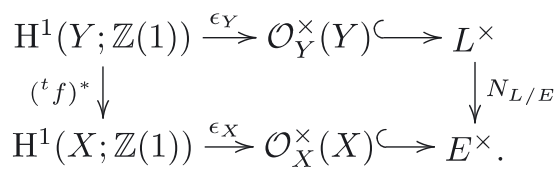

PREUvE: On obtient facilement l'isomorphisme $Z(1) \simeq M\left(\mathrm{G}_{m} /\{1\}\right)[-1]$ ce qui montre que Z(1)[1] est un facteur direct de $M\left(\mathrm{G}_{m}\right)$.

Notons $h^{i}\left(C^{*}\right)$ le $i$-ème préfaisceau de cohomologie d'un complexe motivique $C^{*}$. Si $U$ est un ouvert de $\mathrm{A}_{k}^{1}$ et $X$ un schéma lisse, rappelons que par définition: $\Gamma\left(X ; h^{i}(M(U))\right)=\mathrm{H}_{i}^{\text {sing }}(X \times U / X)$, où $\mathrm{H}_{i}^{\text {sing }}$ désigne l'homologie singulière de Suslin définie dans [SV96]. Dès lors, il résulte de loc.cit., th. 3.1 que le complexe $M\left(\mathrm{G}_{m}\right)$ est concentré en degré 0 et donc Z(1) est concentré en degré 1.

Or, d'après l'isomorphisme canonique $\mathrm{Z}(1)[1] \simeq M\left(\mathrm{~A}_{k}^{1} / \mathrm{G}_{m}\right)$, on obtient une suite exacte courte scindée

$$
0 \rightarrow \Gamma\left(X ; h^{1}(\mathrm{Z}(1))\right) \rightarrow \mathrm{H}_{0}^{\text {sing }}\left(X \times \mathrm{G}_{m} / X\right) \stackrel{(1)}{\longrightarrow} \mathrm{H}_{0}^{\text {sing }}\left(X \times \mathrm{A}_{k}^{1} / X\right) \rightarrow 0 .
$$

Par ailleurs, d'après le théorème cité plus haut, pour tout ouvert $U$ de $\mathbb{A}_{k}^{1}$, il existe un isomorphisme $\mathrm{H}_{0}^{\text {sing }}(X \times U / X) \stackrel{\varepsilon_{X, U}}{\longrightarrow} \operatorname{Pic}\left(X \times \mathbb{P}_{k}^{1}, X \times\left(\mathbb{P}_{k}^{1}-U\right)\right)$. Cet isomorphisme est construit par le procédé suivant: si $\alpha$ est un cycle de $X \times U$ qui est fini équidimensionnel sur $X$, il définit encore un cycle de $X \times \mathbb{P}_{k}^{1}$ (puisque son support est propre sur $X$ ) qui lui-même définit un faisceau inversible $\operatorname{sur} X \times \mathbb{P}_{k}^{1}$. Comme son support est inclus dans $X \times U$, il est canoniquement trivialisé sur $X \times\left(\mathbb{P}_{k}^{1}-U\right)$ et définit donc une classe dans le groupe de Picard relatif.

Le morphisme (1) est induit par l'immersion ouverte évidente. Il correspond donc au morphisme $\operatorname{Pic}\left(\mathbb{P}_{X}^{1}, X_{1} \sqcup X_{\infty}\right) \stackrel{(1)^{\prime}}{\longrightarrow} \operatorname{Pic}\left(\mathbb{P}_{X}^{1}, X_{\infty}\right)$ qui à un faisceau inversible muni d'une trivialisation sur $X_{1} \sqcup X_{\infty}$ associe le même faisceau inversible muni de la trivialisation restreinte à $X_{\infty}$.

Or, utilisant la suite exacte longue du groupe de Picard relatif, le noyau du morphisme précédent est isomorphe à $\mathrm{G}_{m}\left(X_{\infty}\right)$. Cet isomorphisme est construit comme suit: soit $f$ une fonction inversible sur $X_{\infty}$; on peut l'étendre en une fonction $\tilde{f}$ sur $\mathrm{A}_{X}^{1}$; le diviseur de $\tilde{f}$, vu comme fonction rationnelle de $\mathbb{P}_{X}^{1}$, correspond donc à un fibré inversible qui est canoniquement trivialisé sur $X_{1} \sqcup X_{\infty}$; ceci définit une classe dans $\operatorname{Pic}\left(\mathbb{P}_{X}^{1}, X_{1} \sqcup X_{\infty}\right)$ qui ne dépend pas du choix effectué - et qui tombe dans le noyau de (1)' puisque $\tilde{f}$ est inversible $\operatorname{sur} X_{\infty}$. 
On en déduit donc un isomorphisme $\Gamma\left(X ; h^{1}\left(Z_{(1)}(1)\right) \stackrel{\tau_{X}}{\longrightarrow} \mathbb{G}_{m}(X)\right.$. Ceci montre que $h^{1}(Z(1))$ est le premier faisceau de cohomologie de $Z(1)$, et $\tau$ est l'isomorphisme cherché. D'après sa description, le morphisme $\varepsilon_{X, U}$ est compatible au pullback sur $X$, ce qui nous permet de conclure que l'isomorphisme $\tau_{X}$ est naturel en $X$.

Si $f: Y \rightarrow X$ est un morphisme fini équidimensionnel, le morphisme induit par la transposée de $f$ sur $H_{0}^{\text {sing }}(X \times U / X)$, quotient du groupe $\mathrm{c}(X, U)$, correspond au morphisme image directe d'après le lemme 1.1.2. Il résulte de la définition de $\varepsilon_{X, U}$ que ce morphisme correspond au morphisme image directe sur le groupe de Picard relatif. Dès lors, par définition de ce dernier, le morphisme correspondant sur $\mathbb{G}_{m}\left(X_{\infty}\right)$ est bien induit par la norme (cf. [Ful98], prop. 1.4), ce qui conclut la dernière assertion.

REMARQUE 2.2.5. Au cours de la démonstration, nous avons au passage obtenu l'isomorphisme canonique $M \operatorname{Th}\left(\mathrm{A}_{k}^{1}\right)=Z(1)$ [2]. D'après la proposition précédente, le groupe des endomorphismes du motif de Tate est donc égal à:

$$
\operatorname{End}_{D M_{-}^{e f f}(k)}(Z(1))=\operatorname{End}_{D M_{-}^{e f f}(k)}\left(M \operatorname{Th}\left(A_{k}^{1}\right)\right)=Z \text {. }
$$

Cet isomorphisme peut se décrire simplement: pour un morphisme de paires fermées $(f, g):\left(\mathbb{A}_{k}^{1},\{0\}\right) \rightarrow\left(\mathbb{A}_{k}^{1},\{0\}\right)$, l'endomorphisme $M \operatorname{Th}(f, g)$ est envoyé sur le degré de $f$.

Nous verrons toujours $\mathrm{G}_{m}$ comme un faisceau avec transferts avec la structure qui découle de la proposition précédente. Notons que l'on obtient donc $Z(1)=\mathbb{G}_{m}[-1]$. Suivant [MV99], le faisceau avec transferts $\mathbb{G}_{m}$ est appelé la sphère de Tate.

Corollaire 2.2.6. Pour tout schéma lisse $X$, il existe un isomorphisme canonique

$$
\operatorname{Hom}_{D M_{-}^{e f f}(k)}\left(M(X), Z_{A}(1)[2]\right) \stackrel{\tau_{X}}{\longrightarrow} \operatorname{Pic}(X),
$$

qui est naturel par rapport aux morphismes de schémas.

Sif $: Y \rightarrow X$ est un morphisme fini équidimensionnel, le diagramme suivant est commutatif:

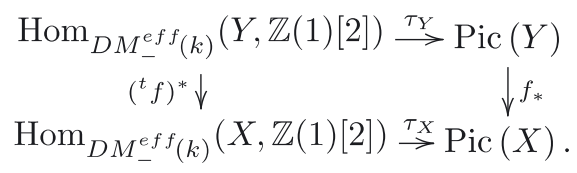


Preuve: D'après ce qui précède et suivant [Voe00b, 3.2.3], on obtient un isomorphisme canonique

$$
\operatorname{Hom}_{D M-(k)}(M(X), Z(1)[2]) \simeq \operatorname{Ext}_{N_{k}^{\text {trt }}}^{1}\left(\mathrm{~L}[X], \mathrm{G}_{m}\right)
$$

qui est naturel en $X$ par rapport aux correspondances finies. D'après [Dég07, 2.9] et le fait que la cohomologie Zariski et Nisnevich du faisceau $\mathrm{G}_{m}$ coïncident, on obtient un isomorphisme

$$
\operatorname{Ext}_{\mathscr{N}_{k}^{\mathrm{tr}}}^{1}\left(\mathrm{~L}[X], \mathrm{G}_{m}\right) \simeq H_{\mathrm{Zar}}^{1}\left(X ; \mathrm{G}_{m}\right) .
$$

Ces deux isomorphismes induisent l'isomorphisme de l'énoncé $\tau_{X}$.

Pour vérifier le second point du corollaire, on introduit la construction suivante: soit $X$ un schéma lisse et $\mathcal{U}=\left(U_{i}\right)_{i=I}$ un recouvrement ouvert fini de $X$; on note $\mathrm{L}[\mathcal{U}]$ le complexe de faisceaux avec transferts à la Čech induit $\operatorname{par} \mathcal{U}$ :

$$
\mathrm{L}[\mathcal{U}]^{n}=\bigoplus_{\underline{\alpha} \in I^{n+1}} \mathrm{~L}\left[U_{\alpha_{1}} \cap \ldots \cap U_{\alpha_{n}}\right],
$$

les différentielles étant données par la somme alternée habituelle. Pour tout entier $i \in \mathbb{N}$, on pose $\check{H}_{t r}^{i}(X ; F)=\lim _{\overrightarrow{U_{X}}} H^{i}\left(\operatorname{Hom}_{\mathscr{N}_{k}^{\mathrm{tr}}}(\mathrm{L}[\mathcal{U}], F)\right)$.

On en déduit un morphisme canonique

$$
\tau_{X}^{\prime}: \check{H}_{t r}^{1}\left(X ; \mathrm{G}_{m}\right) \rightarrow \operatorname{Ext}_{\mathscr{P}_{k}^{\mathrm{tr}}}^{1}\left(\mathrm{~L}\left[X, \mathrm{G}_{m}\right]\right),
$$

qui est un isomorphisme d'après l'isomorphisme obtenu précédemment.

La transposée d'un morphisme fini équidimensionnel définit une fonctorialité covariante sur le groupe de droite. Or on peut construire une fonctorialité covariante sur le groupe de gauche comme suit: soit $f: Y \rightarrow X$ un morphisme fini équidimensionnel entre schémas lisses, et $\mathcal{U} / \mathcal{X}$ un recouvrement ouvert. On considère le recouvrement $f^{-1}(\mathcal{U})$ de $Y$ ainsi que le morphisme induit de recouvrements $\mathfrak{f}: f^{-1}(\mathcal{U}) \rightarrow \mathcal{U}$ dont chaque composante est finie équidimensionnelle. On en déduit d'après 1.1.3 un morphisme de complexes de Čech $\left.\mathrm{L}^{[} \mathfrak{f}\right]: \mathrm{L}[\mathcal{U}] \rightarrow \mathrm{L}\left[f^{-1}(\mathcal{U})\right]$. Comme les recouvrements ouverts de la forme $f^{-1}(\mathcal{U})$ forment une partie cofinale parmi les recouvrements ouverts de $Y$, on obtient par passage à la limite la fonctorialité attendue. Une nouvelle application de 1.1.3 montre que l'isomorphisme $\tau_{X}^{\prime}$ est naturel par rapport aux fonctorialités covariantes que nous venons d'introduire.

Il résulte trivialement de la définition de $\check{H}_{t r}^{1}\left(X ; \mathrm{G}_{m}\right)$ en termes de cocycles que ce groupe est isomorphe au groupe de Picard. Par ailleurs, d'après la proposition précédente, la fonctorialité covariante sur ce groupe 
est induite par des applications norme convenablement choisies. Il résulte enfin de [Ful98, 1.4] que cette fonctorialité coïncide avec le pushout classique sur le groupe de Picard, ce qui permet de conclure.

Nous terminons ce paragraphe par la définition de la classe de Chern motivique qui découle du calcul précédent.

DÉFINITION 2.2.7. On note

$$
\mathfrak{c}_{1}: \operatorname{Pic}(.) \rightarrow \operatorname{Hom}_{D M_{-}^{e f f}(k)}(M(.), Z(1)[2])
$$

l'isomorphisme naturel de préfaisceau sur $\mathscr{L}_{k}$ défini dans le corollaire précédent. Si $X$ est un schéma lisse, et $\lambda \in \operatorname{Pic}(X)$ la classe d'un fibré inversible sur $X$, on note

$$
\mathfrak{c}_{1}(\lambda): M(X) \rightarrow Z(1)[2]
$$

l'image de la transformation naturelle ci-dessus appliquée en $X$ et en $\lambda$, et on l'appelle la classe de Chern motivique de $\lambda$.

Il résulte de l'étude précédente qu'on dispose des relations suivantes pour $X$ et $Y$ deux schémas lisses et $\lambda$ la classe d'un fibré inversible sur $X$ :

(2.2.8) Si $f: Y \rightarrow X$ est un morphisme quelconque, $\mathfrak{c}_{1}\left(f^{*} \lambda\right)=\mathfrak{c}_{1}(\lambda) \circ f_{*}$.

(2.2.9) Si $f: X \rightarrow Y$ est un morphisme fini équidimensionnel,

$$
\mathfrak{c}_{1}\left(f_{*} \lambda\right)=\mathfrak{c}_{1}(\lambda) \circ f^{*} .
$$

\subsection{3 - Motif d'un fibré projectif}

Suivant V.Voevodsky, on associe à tout fibré projectif le morphisme suivant:

DÉfinition 2.2.10. Considérons $X$ un schéma lisse, et $E / X$ un fibré vectoriel sur $X$ de rang $n$. On note $\mathbb{P}(E)$ l'espace projectif induit par $E, \lambda_{E}$ le fibré inversible canonique de $\mathbb{P}(E)$ et $p: \mathbb{P}(E) \rightarrow X$ la projection canonique.

Pour tout entier naturel $r$, on définit le morphisme suivant:

$$
\mathfrak{l}_{r}(E)=\mathfrak{c}_{1}\left(\lambda_{E}\right)^{r} \bigotimes_{\mathrm{P}(E)} p_{*}: M(\mathbb{P}(E)) \rightarrow M(X)(r)[2 r]
$$

où $\mathfrak{c}_{1}\left(\lambda_{E}\right)^{r}$ désigne la puissance $r$-ième du morphisme $\mathfrak{c}_{1}\left(\lambda_{E}\right)$ pour le cupproduit défini dans la remarque 1.2.3.

On posera de plus: $\mathfrak{l}(E)=\sum_{r=0}^{n-1} \mathfrak{l}_{r}(E)$.

La proposition suivante résume la fonctorialité de ce morphisme: 
LEMme 2.2.11. Soient $X$ un schéma lisse et $E / X$ un fibré vectoriel.

(1) Soient $F / X$ un fibré vectoriel et $\sigma: E \rightarrow F$ un isomorphisme de fibrés sur $X$. Alors, notant $\bar{\sigma}: \mathbb{P}(E) \rightarrow \mathbb{P}(F)$ l'isomorphisme induit par $\sigma$, $\mathfrak{l}(E) \circ \bar{\sigma}_{*}=\mathfrak{l}(F)$.

(2) Soient $Y$ un schéma lisse et $f: Y \rightarrow X$ un morphisme de schémas. Posons $E_{Y}=E \times_{X} Y$ et notons $\bar{f}: \mathbb{P}\left(E_{Y}\right) \rightarrow \mathbb{P}(E)$ le morphisme induit par $f$. Alors, pour tout $r \in \mathbb{N}, \mathfrak{l}_{r}(E) \circ g_{*}=f_{*}(r)[2 r] \circ \mathfrak{l}_{r}\left(E_{Y}\right)$.

(3) Considérons les hypothèses du numéro précédent et supposons que $f$ est fini équidimensionnel. Alors, $\mathfrak{l}_{r}\left(E_{Y}\right) \circ g^{*}=f^{*}(r)[2 r] \circ \mathfrak{l}_{r}(E)$.

PReuve: Pour les deux premières assertions, il nous suffit d'appliquer la formule 2.2.8 pour la compatibilité des classes de Chern au pullback et le deuxième point de 1.2.4 pour celle du cup-produit.

Pour la dernière assertion, on note $\lambda$ (resp. $\lambda_{Y}$ ) le fibré en droite canonique sur $\mathbb{P}(E)$ (resp. $\mathrm{P}\left(E_{Y}\right)$ ), et $\Delta$ (resp. $\Delta^{\prime}$ ) le morphisme diagonal de $\mathbb{P}(E)$ (resp. $\mathbb{P}\left(E_{Y}\right)$ ).

On trace le diagramme suivant:

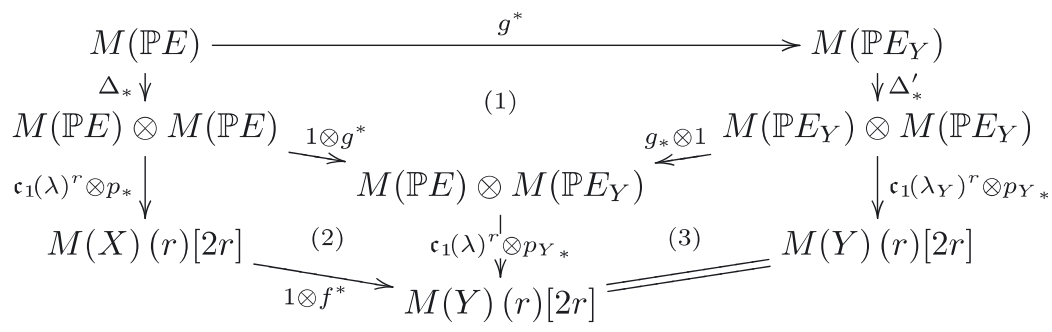

Le diagramme (1) est commutatif d'après 1.2.4, le diagramme (2) est commutatif d'après 1.1.4 et le diagramme (3) est commutatif d'après 2.2.8.

La motivation pour introduire la définition précédente est la proposition suivante (cf. [Voe00b, 3.5.1]):

Proposition 2.2.12 [Voevodsky]. Soient X un schéma algébrique lisse et $E / X$ un fibré vectoriel de rang $n>0$.

Alors, le morphisme $\mathfrak{l}(E): M(\mathbb{P}(E)) \rightarrow \bigoplus_{r=0}^{n-1} M(X)(r)[2 r]$ de la définition 2.2.10 est un isomorphisme.

REMARQUE 2.2.13. Suivant la méthode classique en cohomologie, on peut déduire de cet isomorphisme des classes de Chern motiviques - vues comme morphismes entre motifs - qui prolongent les classes de la forme 
$\mathfrak{c}_{1}(\lambda)$ définies en 2.2.7. On déduit du lemme 2.2.11 la fonctorialité standard de ces classes de Chern. Toutefois, nous ne les utiliserons pas.

\subsection{4 - Isomorphisme de Thom}

Soient $X$ un schéma lisse et $E / X$ un fibré vectoriel de rang $n$. Dans ce qui suit, nous explicitons le motif de Thom de $E / X$.

Posons $\hat{E}=E \times_{X} \mathrm{~A}_{X}^{1}$ muni de sa structure de fibré vectoriel sur $X$. Alors, on obtient les isomorphismes suivants:

$$
\begin{aligned}
M \operatorname{Th}(E) & =M(E, X) \stackrel{(1)}{\simeq} M(\mathbb{P}(\hat{E}), X) \\
& =M(\mathbb{P}(\hat{E}) / \mathbb{P}(\hat{E})-X) \stackrel{(2)}{\simeq} M(\mathbb{P}(\hat{E}) / \mathbb{P}(E)) .
\end{aligned}
$$

Le premier isomorphisme est induit par l'immersion ouverte $E \rightarrow \mathbb{P}(\hat{E})$ d'après la propriété d'excision de la topologie de Nisnevich (cf. [Dég04, 2.3]) et le deuxième résulte du fait que $\mathbb{P}(\hat{E})-X$ est un fibré vectoriel sur $\mathbb{P}(E)$.

On déduit de cet isomorphisme un triangle distingué canonique

$$
M(\mathbb{P}(E)) \stackrel{i_{*}}{\longrightarrow} M(\mathbb{P}(\hat{E})) \stackrel{\pi_{E}}{\longrightarrow} M \operatorname{Th}(E) \stackrel{+1}{\longrightarrow}
$$

où le morphisme $i$ est l'immersion fermée correspondant à l'hyperplan à l'infini.

Or, d'après le deuxième point du lemme 2.2.11, le morphisme $i_{*}$ correspond à travers les isomorphismes $\mathfrak{l}(E)$ et $\mathfrak{l}(\hat{E})$ à l'injection canonique des $n$ premiers facteurs de $\bigoplus_{r=0}^{n} M(X)(r)[2 r]$. Il en résulte que le triangle cidessus est scindé et que le morphisme suivant

$$
M(X)(n)[2 n] \hookrightarrow \bigoplus_{r=0}^{n} M(X)(r)[2 r] \stackrel{\Upsilon(\hat{E})^{-1}}{\longrightarrow} M(\mathbb{P}(\hat{E})) \stackrel{\pi_{E}}{\longrightarrow} M \operatorname{Th}(E)
$$

est un isomorphisme.

DÉfinition 2.2.14. Avec les notations et hypothèses qui précédent, on appelle isomorphisme de Thom et on note $\theta(E)$ la réciproque de l'isomorphisme ci-dessus.

L'étude détaillée du motif de Tate qui précède et de la classe de Chern motivique d'ordre 1 se justifie dans la mesure où l'on prouve la fonctorialité suivante de l'isomorphisme de Thom: 
LEMME 2.2.15. Soient $X$ un schéma lisse et $E / X$ un fibré vectoriel de $\operatorname{rang} n$.

(1) Soit $F / X$ est un fibré vectoriel sur $X$ et $\sigma: E \rightarrow F$ un isomorphisme de fibrés sur $X$. Alors, $\theta(E) \circ\left(\sigma, 1_{X}\right)_{*}=\theta(F)$.

(2) Soient $Y$ un schéma lisse etf $: Y \rightarrow X$ un morphisme de schémas. Posons $E_{Y}=E \times_{X} Y$ et notons $f_{E}: E_{Y} \rightarrow E$ le morphisme induit par $f$.

Alors, $\theta(E) \circ\left(f_{E}, f\right)_{*}=f_{*}(n)[2 n] \circ \theta\left(E_{Y}\right)$.

(3) Considérons les hypothèses ci-dessus et supposons que f est fini équidimensionnel. Alors, $\theta\left(E_{Y}\right) \circ\left(f_{E}, f\right)^{*}=f^{*}(n)[2 n] \circ \theta(E)$.

PREUVE: Dans chaque cas, on se ramène en utilisant le triangle distingué (B) à montrer l'assertion analogue où l'on remplace $\theta(E)$ (resp. $\left.\theta\left(E_{Y}\right)\right)$ par $\mathfrak{l}(E)$ (resp. $\left.\mathfrak{l}\left(E_{Y}\right)\right)$. Dès lors, on est ramené au lemme 2.2.11.

Soient $X$ un schéma lisse, $E / X$ un fibré vectoriel de rang 1 et $r$ un entier naturel. On considère le dual $\mathcal{E}$ du faisceau des sections de $E / X$.

Notons $\mathcal{S}_{*}(\mathcal{E})$ l'algèbre symétrique de $\mathcal{E}$. Le morphisme d'inclusion canonique $\mathcal{E}^{r} \rightarrow \mathcal{S}_{*}(\mathcal{E})$ induit un morphisme homogène de degré $r$ de $\mathcal{O}_{X}$-algèbres graduées $\mathcal{S}_{*}\left(\mathcal{E}^{r}\right) \rightarrow \mathcal{S}_{*}(\mathcal{E})$.

On pose $E^{(r)}=\operatorname{Spec}_{\mathrm{X}}\left(\mathcal{S}\left(\mathcal{E}^{\mathrm{r}}\right)\right)$, et on note $\mu: E \rightarrow E^{(r)}$ le spectre du morphisme précédent; c'est donc un morphisme homogène de degré $r$ de fibrés vectoriels sur $X$.

On a ainsi obtenu un morphisme fini équidimensionnel de paires fermées $\left(\mu, 1_{X}\right):(E, X) \rightarrow\left(E^{(r)}, X\right)$ (ce morphisme n'est pas cartésien si $r>1)$.

Proposition 2.2.16. On adopte les hypothèses et notations introduites ci-dessus.

Alors, les diagrammes suivants sont commutatifs:
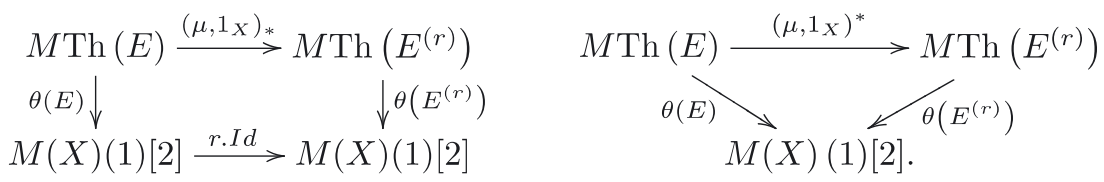

PREUVE: On note $\lambda$ et $\lambda^{\prime}$ les fibrés inversibles canoniques respectifs sur $\mathbb{P}(\hat{E})$ et $\mathbb{P}\left(\hat{E}^{(r)}\right)$. On note $v: \mathbb{P}(\hat{E}) \rightarrow \mathbb{P}\left(\hat{E}^{(r)}\right)$ le morphisme induit par $\hat{\mu}: \hat{E} \rightarrow \hat{E}^{(r)}$, obtenu suivant le procédé décrit ci-dessus. 
On s'intéresse tout d'abord au diagramme de gauche. On pose $\mathcal{F}=\mathcal{E} \oplus \mathcal{O}_{X}[t]$. Tout d'abord, on a le calcul facile suivant:

$$
\bigoplus_{i>0} \mathcal{F}^{r i} \otimes_{\mathcal{S}_{*}(\mathcal{F})} \bigoplus_{j \geq 0} \mathcal{F}^{j}=\bigoplus_{i>0} \bigoplus_{0 \leq j<r} \mathcal{F}^{r i+j}
$$

Autrement dit, $v^{*}\left(\lambda^{\prime}\right)=\lambda^{r}$, ce qui implique $v^{*} c_{1}\left(\lambda^{\prime}\right)=r . c_{1}(\lambda)$.

Dès lors, utilisant la formule 2.2.8, le morphisme

$$
\varphi=\mathfrak{l}\left(\hat{E}^{(r)}\right) \circ v_{*} \circ \mathfrak{l}(\hat{E})^{-1}: M(X) \oplus M(X)(1)[2] \rightarrow M(X) \oplus M(X)(1)[2]
$$

a pour matrice $\left(\begin{array}{cc}I d & 0 \\ 0 & r . I d\end{array}\right)$, ce qui permet de conclure par définition de l'isomorphisme de Thom.

Considérons maintenant l'autre diagramme. On note $p$ et $p^{\prime}$ les projections respectives de $\mathbb{P}(\hat{E})$ et $\mathbb{P}\left(\hat{E}^{(r)}\right)$ sur $X$.

Alors, on obtient l'égalité suivante de morphismes de complexes motiviques: $p_{*} v^{*}=p_{*}^{\prime} v_{*} v^{*} \stackrel{(1)}{=} p_{*}^{\prime}(\operatorname{deg}(v) \cdot I d)=r \cdot p_{*}^{\prime}$. L'égalité (1) résulte de la formule de projection de 1.1.4.

Par ailleurs, $v$ est égal à l'identité sur l'hyperplan à l'infini de $\mathrm{P}(\hat{E})$ et $\mathbb{P}\left(\hat{E}^{(r)}\right)$ lorsqu'on l'a identifié à $X$. Cela implique $v_{*}\left(c_{1}\left(\lambda^{\prime}\right)\right)=c_{1}(\lambda)$ dans le groupe de Picard, ce qui se traduit grâce à la formule 2.2.9 par l'égalité $\mathfrak{c}_{1}\left(\lambda^{\prime}\right) \circ v^{*}=\mathfrak{c}_{1}(\lambda)$ concernant les classes de Chern motiviques. Appliquant enfin la formule de projection 1.2.4, on obtient $\left(p_{*} \bigotimes \mathfrak{c}_{1}(\lambda)\right) \circ v^{*}=p_{*}^{\prime} \bigotimes \mathfrak{c}_{1}\left(\lambda^{\prime}\right)$.

On en déduit que la matrice du morphisme

$$
\psi=\mathfrak{l}(\hat{E}) \circ v^{*} \circ \mathfrak{l}\left(\hat{E}^{(r)}\right)^{-1}: M(X) \oplus M(X)(1)[2] \rightarrow M(X) \oplus M(X)(1)[2]
$$

est égale à la matrice diagonale $\left(\begin{array}{cc}r . I d & 0 \\ 0 & I d\end{array}\right)$.

De nouveau, le diagramme commutatif suivant permet donc de conclure

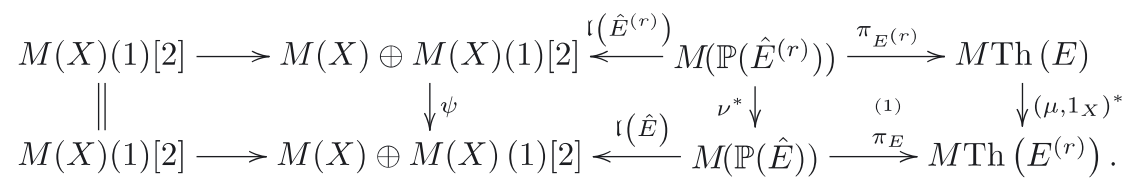

La commutativité du carré (1) résulte de la définition de l'isomorphisme qui sert à construire le triangle distingué (B) et de la formule de 1.1.4. 
2.2.5-Conclusion

Soit $(X, Z)$ une paire lisse de codimension $n$.

Considérons les morphismes de déformation au cône normal:

$$
(X, Z) \stackrel{d}{\longrightarrow}\left(D_{Z} X, \mathrm{~A}_{Z}^{1}\right) \stackrel{d^{\prime}}{\longleftarrow}\left(N_{Z} X, Z\right) .
$$

Nous associons à $(X, Z)$ l'isomorphisme de pureté:

$$
\mathfrak{p}_{X, Z}: M(X, Z) \stackrel{d_{*}}{\longrightarrow} M\left(D_{Z} X, \mathrm{~A}_{Z}^{1}\right) \stackrel{\left(d_{*}^{\prime}\right)^{-1}}{\longrightarrow} M \operatorname{Th}\left(N_{Z} X\right) \stackrel{\theta\left(N_{Z} X\right)}{\longrightarrow} M(Z)(n)[2 n] .
$$

Remarque 2.2.17. Nous dégageons explicitement cet isomorphisme car c'est en élucidant sa fonctorialité que nous obtenons les résultats de la section suivante.

\section{3 - Définition}

On introduit maintenant le triangle de Gysin qui apparaît déjà dans [VSF00], chap. 5:

DÉFInition 2.3.1. Soit $(X, Z)$ une paire lisse de codimension $d$. On note $i: Z \rightarrow X$ et $j: X-Z \rightarrow X$ les immersions canoniques.

On définit à partir du triangle distingué (A) et grâce à l'isomorphisme 2.2.5 le triangle distingué

$$
M(X-Z) \stackrel{j_{*}}{\longrightarrow} M(X) \stackrel{i^{*}}{\longrightarrow} M(Z)(d)[2 d] \stackrel{\partial_{X, Z}}{\longrightarrow} M(X-Z)[1]
$$

appelé triangle de Gysin. On dit que $i^{*}$ est le morphisme de Gysin associé à $i$ et que $\partial_{X, Z}$ est le résidu associé à la paire $(X, Z)$.

REMARque 2.3.2. La terminologie «résidu» est particulièrement motivée par la définition 5.4.6.

\section{4 - Fonctorialité covariante}

Dans ce qui suit, on étudie la fonctorialité du triangle de Gysin. Rappelons en effet que l'on a associé à un morphisme $(f, g):(Y, T) \rightarrow(X, Z)$ de paires fermées, le morphisme de motifs relatifs

$$
(f, g)_{*}: M_{T}(Y) \rightarrow M_{Z}(X)
$$


de telle manière que ce morphisme s’insère dans un morphisme évident de triangles distingués du type (A).

DÉfinition 2.4.1. Soit $(f, g):(Y, T) \rightarrow(X, Z)$ un morphisme de paires fermées. On suppose que $Z$ (resp. $T$ ) est connexe et lisse sur $k$ de codimension $n$ dans $X$ (resp. $m$ dans $Y$ ).

On définit alors un morphisme $(f, g)$ ! par le diagramme commutatif suivant:

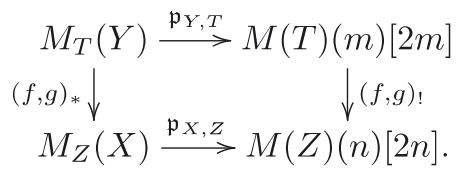

Reprenons les notations de cette définition. Considérons les immersions canoniques $i: Z \rightarrow X, j: X-Z \rightarrow X, k: T \rightarrow Y$ et $l: Y-T \rightarrow T$. Par définition, le morphisme $(f, g)$ ! fait donc partie du morphisme suivant de triangles de Gysin:

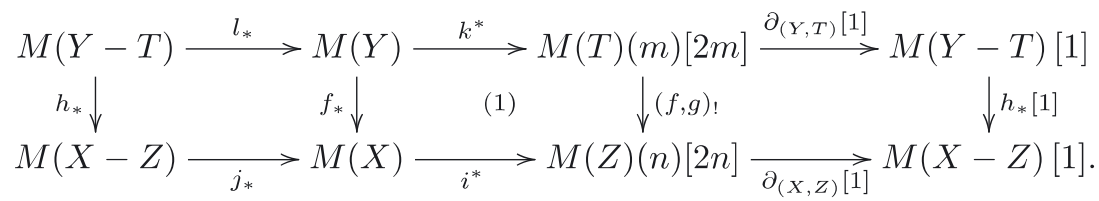

REMARQUE 2.4.2. La commutativité du carré (1) justifie notre choix de notation pour le morphisme $(f, g)$ ! que l'on considère comme un analogue du morphisme de Gysin raffiné suivant la terminologie de [Ful98], §6.2 (voir aussi th. 6.2 (a)). Plus précisément, dans le cas où $(f, g)$ est cartésien, on peut voir que le morphisme $(f, g)$ ! induit en cohomologie motivique de degré $2 n$ et de poids $n$ - qui est isomorphe au groupe de Chow des cycles de codimension $n$ - le morphisme de Gysin raffiné défini par Fulton relativement au carré cartésien $(f, g)$.

\subsection{1 - Transversalité}

Rappelons avant d'énoncer la proposition qui nous intéresse la définition suivante (cf. [EGA4], 17.13.3):

DÉfinition 2.4.3. Soit $(X, Z)$ une paire fermée lisse de codimension $n$.

Soient $Y$ un schéma lisse et $f: Y \rightarrow X$ un morphisme de schémas. On dit que $f$ est transverse à $Z$ si le schéma $Z \times_{X} Y$ est lisse purement de codimension $n$ dans $Y$. 
Proposition 2.4.4. Soit $(X, Z)$ une paire lisse de codimension $n$.

Soit $f: Y \rightarrow X$ un morphisme. On pose $T=Z \times_{X} Y$ et on note $g: T \rightarrow Z$ le morphisme induit par $f$.

Alors, sif est transverse à $Z,(f, g)_{!}=g_{*}(n)[2 n]$.

Preuve: Par fonctorialité de la déformation au cône normal, le morphisme $(f, g)_{*}$ correspond à travers les isomorphismes de déformation au cône normal, au morphisme $\left(N_{g} f, g\right)_{*}: M \operatorname{Th}\left(N_{T} Y\right) \rightarrow M \operatorname{Th}\left(N_{Z} X\right)$ où $N_{g} f$ est défini comme la composée des morphismes du diagramme ci-dessous:

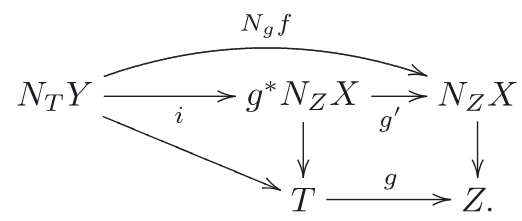

Le morphisme $i$ est en général une immersion fermée, mais comme $f$ est transverse à $Z, \operatorname{codim}_{X} Z=\operatorname{codim}_{Y} T$. On en déduit que le monomorphisme de fibrés vectoriels $i: N_{T} Y \rightarrow g^{*}\left(N_{Z} X\right)$ est en fait un isomorphisme.

Dès lors, le diagramme suivant est commutatif:

$$
\begin{aligned}
& \begin{array}{c}
M \operatorname{Th}\left(N_{T} Y\right) \underset{\left(i, 1_{X}\right)_{*}}{\longrightarrow} \operatorname{MTh}\left(g^{*} N_{Z} X\right) \stackrel{\left(N_{g} f, g\right)_{*}}{\underset{\left(g^{\prime}, g\right)_{*}}{\longrightarrow}} \\
\theta\left(g_{T}^{*} N_{Z} X\right)
\end{array} \\
& M(T)(n)[2 n]=M(T)(n)[2 n] \underset{g_{*}(n)[2 n]}{=} M(Z)(n)[2 n],
\end{aligned}
$$

d'après les deux premiers points du lemme 2.2.15.

\subsection{2 - Ramification en codimension 1}

Rappelons qu'un épaississement de schémas est une immersion fermée qui est un homéomorphisme sur les espaces topologiques sous-jacents.

DÉFInITION 2.4.5. Considérons un diagramme commutatif dans la catégorie des schémas

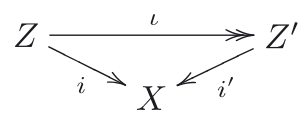

dans lequel chaque morphisme est une immersion fermée et $l$ est un épaississement. Notons $\mathcal{I}$ (resp. $\mathcal{I}^{\prime}$ ) l'idéal de $i$ (resp. $i^{\prime}$ ). 
(1) Soit $\alpha$ un entier naturel. On dira que l'épaississement $\imath$ est exact d'ordre $\alpha$ dans $X$ si $\mathcal{I}^{\prime}=\mathcal{I}^{\alpha}$.

(2) Plus généralement, on dira que l'épaississement $\imath$ est exact dans $X$ si pour toute composante irréductible $Z_{\lambda}^{\prime}$ de $Z^{\prime}$, notant $Z_{\lambda}$ la composante irréductible de $Z$ qui correspond à $Z^{\prime}$, il existe un entier $\alpha_{\lambda}$ tel que le morphisme induit par restriction $l_{\lambda}: Z_{\lambda} \rightarrow Z_{\lambda}^{\prime}$ soit exact d'ordre $\alpha_{\lambda}$ dans $X$.

Remarque 2.4.6. Dans la deuxième partie de la définition, la suite d'entiers $\alpha_{\lambda}$ où $\lambda$ parcourt les composantes irréductibles de $Z^{\prime}$ est unique. En effet, pour toute composante irréductible $Z_{\lambda}^{\prime}$ de $Z^{\prime}$ de point générique $z_{\lambda}^{\prime}$, si l'on note $Z_{\lambda}$ la composante irréductible de $Z$ qui lui correspond ainsi que $z_{\lambda}$ son point générique, on a $\alpha_{\lambda}=\lg \left(\mathcal{O}_{Z_{\lambda}^{\prime}, z_{\lambda}^{\prime}}\right)-\lg \left(\mathcal{O}_{Z_{\lambda}, z_{\lambda}}\right)+1$.

Munis de cette définition, nous pouvons introduire la condition voulue sur les morphismes de paires fermées:

DÉfINITION 2.4.7. Soit $(f, g):(Y, T) \rightarrow(X, Z)$ un morphisme de paires fermées.

On dit que $(f, g)$ est exact si le morphisme canonique $T \rightarrow Z \times_{Y} X$ est un épaississement exact dans $Y$. Si $T_{\lambda}$ est une composante irréductible de $T$, on appellera ordre de $(f, g)$ en $T_{\lambda}$ l'ordre de l'épaississement correspondant en $T_{\lambda}$.

REMARQue 2.4.8. Cette propriété des morphismes de paires fermées est stable par changement de base mais pas par composition.

2.4.9. Soit $(f, g):(Y, T) \rightarrow(X, Z)$ un morphisme exact de paires fermées où $T$ est réunion disjointe de ses composantes connexes. On cherche à définir un morphisme de paires fermées

$$
\left(D_{g} f, 1 \times g\right):\left(D_{T} Y, \mathrm{~A}_{T}^{1}\right) \rightarrow\left(D_{Z} X, \mathbb{A}_{Z}^{1}\right)
$$

qui coïncide avec $(f, g)$ sur la fibre au-dessus de 1 .

Si l'on pose $T^{\prime}=Z \times_{X} Y$, on a par définition une décomposition $\mathrm{du}$ morphisme $(f, g)$ en le diagramme

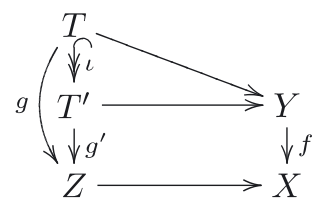

où $\imath$ est un épaississement exact par rapport à $Y$. 
Le morphisme $\left(f, g^{\prime}\right)$ étant cartésien, il nous reste à construire un morphisme sur les espaces de déformation pour le triangle du haut. On est donc réduit au morphisme exact $\left(1_{Y}, l\right):(Y, T) \rightarrow\left(Y, T^{\prime}\right)$.

Commençons par supposer que $T$ est irréductible. On note $\mathcal{I}$ (resp. $\mathcal{I}^{\prime}$ ) l'idéal de $T$ (resp. $T^{\prime}$ ) dans $Y$, et on note $\alpha$ l'ordre de l'épaississement exact $l$. Par définition, c'est l'unique entier $\alpha$ tel que $\mathcal{I}^{\prime}=\mathcal{I}^{\alpha}$.

Rappelons que l'espace de déformation $D_{T} Y$ est le spectre de la $\mathcal{O}_{Y^{-}}$ algèbre cohérente $\bigoplus_{n \in Z}(\mathcal{I})^{n} \cdot t^{-n}$. Dès lors, on peut définir simplement un morphisme sur les espaces de déformation:

$$
\begin{aligned}
\bigoplus_{n \in \mathrm{Z}}\left(\mathcal{I}^{\prime}\right)^{n} \cdot t^{-n} & \rightarrow \bigoplus_{m \in Z}(\mathcal{I})^{m} \cdot t^{-m} \\
x \cdot t^{-n} & \mapsto x \cdot t^{-\alpha n}
\end{aligned}
$$

puisque toute section $x$ de $\left(\mathcal{I}^{\prime}\right)^{n}$ est aussi une section de $(\mathcal{I})^{\alpha n}$. En particulier, ce morphisme est l'élévation à la puissance $\alpha$ sur le paramètre de la déformation, et il définit un morphisme $D_{l}: D_{T} Y \rightarrow D_{T^{\prime}} Y$. Il en résulte que $D_{l}$ induit un morphisme $D_{l}^{\prime}: \mathbb{A}_{T}^{1} \rightarrow \mathbb{A}_{T^{\prime}}^{1}$ égal à $\tau^{\alpha} \times \imath$ où $\tau^{\alpha}: \mathbb{A}_{k}^{1} \rightarrow \mathbb{A}_{k}^{1}$ est l'élévation à la puissance $\alpha$.

Ainsi, on en déduit un morphisme exact d'ordre $\alpha\left(D_{l}, D_{l}^{\prime}\right):\left(D_{T} Y, \mathrm{~A}_{T}^{1}\right) \rightarrow$ $\rightarrow\left(D_{T^{\prime}} Y, \mathrm{~A}_{T^{\prime}}^{1}\right)$.

On calcule par ailleurs les fibres en 0 et 1 de cette construction pour arriver au diagramme de paires fermées suivant:

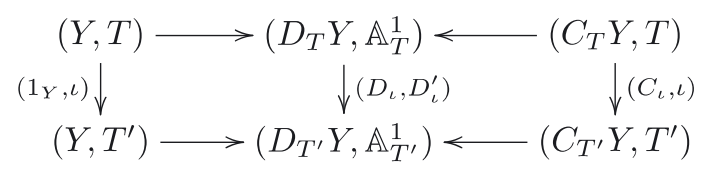

dans lequel les morphismes horizontaux sont les morphismes de déformation au cône normal - tous cartésiens fermés - et les morphismes verticaux sont exacts d'ordre $\alpha$; le morphisme $C_{l}$ est par définition le spectre du morphisme

$$
\begin{aligned}
\bigoplus_{n \in \mathbb{N}}\left(\mathcal{I}^{\prime}\right)^{n} /\left(\mathcal{I}^{\prime}\right)^{n+1}=\bigoplus_{n \in \mathbb{N}} \mathcal{I}^{\alpha n} / \mathcal{I}^{\alpha n+\alpha} & \stackrel{(1)}{\longrightarrow} \bigoplus_{n \in \mathbb{N}} \mathcal{I}^{\alpha n} / \mathcal{I}^{\alpha n+1} \\
& \stackrel{(2)}{\longrightarrow} \bigoplus_{m \in \mathbb{N}} \mathcal{I}^{m} / \mathcal{I}^{m+1},
\end{aligned}
$$

où le morphisme (1) est l'épimorphisme canonique, et (2) est le monomorphisme canonique correspondant à l'inclusion du facteur direct.

Autrement dit, d'un point de vue géométrique, le morphisme $C_{l}$ se factorise comme ci-dessous 


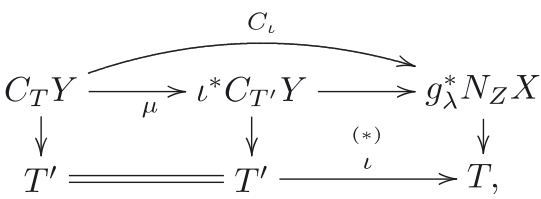

où le carré $(*)$ est cartésien, et le morphisme $\mu$ est dominant de degré $\alpha$.

Ce calcul montre enfin que le morphisme canonique $T^{\prime} \times_{C_{T^{\prime}} Y} C_{T} Y \rightarrow T^{\prime}$ est un isomorphisme, et donc que $\left(C_{l}, l\right)$ est bien un morphisme exact.

Dans le cas général, comme $T^{\prime \prime}$ est réunion disjointe de ses composantes irréductibles notées $\left(T_{\lambda}^{\prime}\right)_{\lambda}$, on pose $D_{l}=\sum_{\lambda} D_{l_{\lambda}}$ où $l_{\lambda}$ est l'épaississement canonique correspondant à $T_{\lambda}^{\prime}$.

Ceci nous permet donc de définir le morphisme annoncé à partir du morphisme exact $(f, g)$ en considérant le diagramme commutatif

(C)

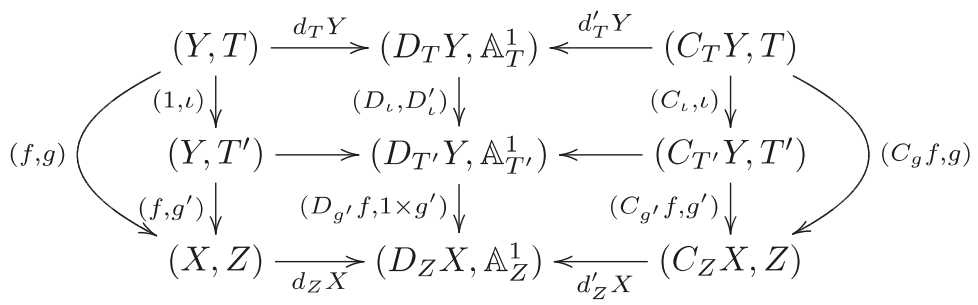

et en posant: $D_{g} f=D_{g^{\prime}} f \circ D_{l}$ et $C_{g} f=C_{g^{\prime}} f \circ C_{l}$.

Ayant ainsi étendu la fonctorialité de la déformation au cône normal pour les morphismes exacts, nous pouvons aborder la démonstration du théorème suivant:

ThÉORÈme 2.4.10. Soient $(X, Z)$ et $(Y, T)$ deux paires fermées lisses de codimension 1 telles que $Z$ est connexe. Soit $(f, g):(Y, T) \rightarrow(X, Z)$ un morphisme exact.

Posons $T^{\prime}=Z \times_{X} Y$. Pour tout $t \in T^{(0)}$, on pose $r_{t}=\lg \left(\mathcal{O}_{T^{\prime}, t}\right)+1$ où $t$ est considéré comme un point de $T^{\prime}$, et on note $g_{t}$ la restriction de g à la composante irréductible de $T$ de point générique $t$.

Alors, $(f, g)_{!}=\sum_{t \in T^{(0)}} r_{t} \cdot g_{t_{*}}(1)[2]$.

Remarque 2.4.11. Si $T$ est irréductible, l'entier $r$ est l'indice de ramification de l'idéal maximal de $\mathcal{O}_{Y, T}$ dans l'extension $\kappa(T) / \kappa(Z)$ au sens de [Ser68], où l'anneau de valuation discrète $\mathcal{O}_{X, Z}\left(\operatorname{resp} . \mathcal{O}_{Y, T}\right)$ est l'anneau des entiers de $\kappa(Z)(\operatorname{resp} . \kappa(T))$. 
PreUve: On peut supposer que $T$ est connexe et on pose $r=r_{t}$ où $t$ désigne l'unique point générique de $T$.

On utilise la fonctorialité construite avant l'énoncé du théorème. Adoptant les notations introduites dans cette construction, $(f, g)_{*}$ est isomorphe à travers les morphismes de déformation au cône normal au morphisme $\left(N_{g} f, g\right)_{*}$. Rappelons que le morphisme $N_{g} f$ est défini par le diagramme suivant:

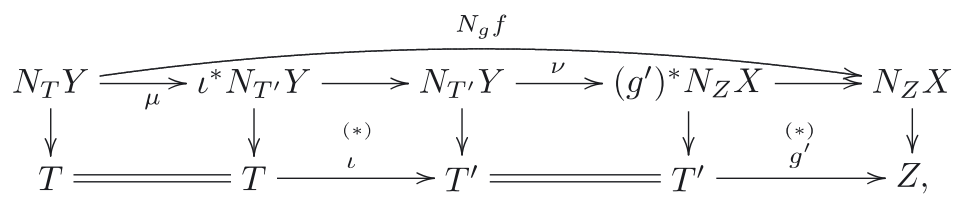

dans lequel les carrés $(*)$ sont cartésiens.

Or, le morphisme $v$ est une immersion fermée entre fibrés vectoriels de rang 1 . C'est donc un isomorphisme.

Par ailleurs, notant $\mathcal{I}$ l'idéal de $T$ dans $Y$,

$$
\imath^{*}\left(N_{T^{\prime}} Y\right)=\operatorname{Spec}_{T}\left(\bigoplus_{n \in \mathbb{N}} \mathcal{I}^{m} / \mathcal{I}^{m+1}\right)
$$

et le morphisme $\mu$ est induit par le morphisme de $\mathcal{O}_{T}$-modules cohérents

$$
\bigoplus_{n \in \mathbb{N}} \mathcal{I}^{m} / \mathcal{I}^{m+1} \rightarrow \bigoplus_{m \in \mathbb{N}} \mathcal{I}^{m} / \mathcal{I}^{m+1}
$$

qui est l'inclusion canonique.

On peut donc lui appliquer la proposition 2.2.16; on en déduit que le diagramme suivant est commutatif:

$$
\begin{gathered}
M \operatorname{Th}\left(N_{T} Y\right) \stackrel{M\left(\mu, 1_{T}\right)}{\longrightarrow} M \operatorname{Th}\left(\iota^{*} N_{T^{\prime}} Y\right) \\
\forall\left(N_{T} Y\right) \downarrow \\
\quad \downarrow(T)(1)[2] \stackrel{r . I d}{\longrightarrow} M(T)(1)[2],
\end{gathered}
$$

ce qui termine la démonstration d'après les deux premiers points du lemme 2.2.15.

\section{5 - Fonctorialité contravariante}

Soit $(f, g):(Y, T) \rightarrow(X, Z)$ un morphisme fini équidimensionnel de paires fermées lisses de codimension $n$. Dans 2.1.2, on a défini un morphisme $(f, g)^{*}: M(X, Z) \rightarrow M(Y, T)$. A travers les isomorphismes de 
pureté 2.2.5, ce morphisme correspond à un morphisme noté

$$
(f, g)^{!}: M(Z)(n)[2 n] \rightarrow M(T)(n)[2 n] .
$$

Par définition, ce dernier s’inscrit dans le morphisme de triangles distingués:

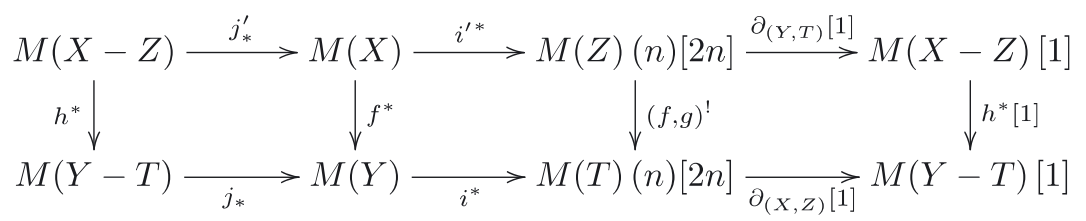

où $g$ et $h$ sont les morphismes induits par $f$.

REMARQUE 2.5.1. Le morphisme $(f, g)^{!}$est compatible au produit de composition des morphismes de paires fermées. Plus généralement, soit $(X, Z)$ une paire fermée lisse. Alors $Z=\sqcup_{i \in I} Z_{i}$ où $Z_{i}$ parcourt les composantes irréductibles de $Z$. Pour tout $i \in I$, notons $n_{i}$ la codimension de $Z_{i}$ dans $X$. Nous avons associé à $(X, Z)$ le motif $\oplus_{i \in I} M\left(Z_{i}\right)\left(n_{i}\right)\left[2 n_{i}\right]$. D'après ce qui précède, cette association est fonctorielle contravariante par rapport aux morphismes de paires fermées finis équidimensionnels. D’après la sous-section précédente, elle est aussi fonctorielle covariante par rapport aux morphismes de paires fermées.

De plus, la proposition 2.1.4 montre que ces deux types de morphismes satisfont une formule de projection généralisée. Il suffit de remplacer les symboles $*$ qui apparaissent dans 2.1.4 par des symboles ! pour obtenir le bon énoncé. La démonstration est évidente.

Proposition 2.5.2. Soient $(X, Z)$ et $(Y, T)$ deux paires fermées lisses de codimension $n$, et $(f, g):(Y, T) \rightarrow(X, Z)$ un morphisme fini équidimensionnel.

On suppose l'une des deux conditions suivantes vérifiée:

(1) Le morphisme $(f, g)$ est cartésien.

(2) Le morphisme $(f, g)$ est exact, et $n=1$.

Alors, $(f, g)^{!}=g^{*}(n)[2 n]$.

Preuve: On se place dans le cas de la première hypothèse. Tout d'abord, le morphisme $D_{g} f$ (resp. $N_{g} f$ ) est fini équidimensionnel. Dès lors, utilisant la fonctorialité naturelle de la déformation au cône normal par rapport aux morphismes cartésiens de paires fermées, la proposition 2.1.4 
entraîne que le morphisme $(f, g)^{*}$ est isomorphe par déformation au cône normal au morphisme $\left(N_{g} f, g\right)^{*}$.

Comme on l'a déjà vu, le morphisme canonique $i: N_{T} Y \rightarrow g^{*} N_{Z} X$ est un isomorphisme. Si on note $p: g^{*} N_{Z} X \rightarrow N_{Z} X$ le morphisme induit par $g$, on obtient le diagramme commutatif suivant

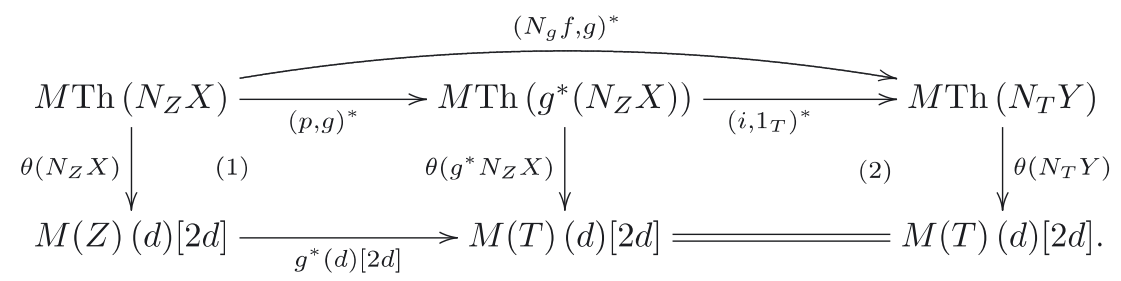

En effet, la partie (1) est commutative d'après le dernier point du lemme 2.2.15. La partie (2) est de même commutative d'après le premier point de ce lemme, puisqu'on déduit l'égalité $\left(i, 1_{T}\right)^{*}=\left(i, 1_{T}\right)_{*}^{-1}$ de la proposition 2.1.4 et du fait que $i$ est un isomorphisme. Ceci conclut la proposition dans le cas de la première hypothèse.

Plaçons nous dans le cas de la seconde hypothèse. Tout d'abord, comme précédemment, le morphisme $(f, g)^{*}$ est isomorphe par déformation au cône normal au morphisme $\left(N_{g} f, g\right)^{*}$ où le morphisme $N_{g} f: N_{T} Y \rightarrow N_{Z} X$ a été défini dans 2.4 .9 (il apparaitra dans ce qui suit que ce dernier morphisme est effectivement fini équidimensionnel).

Posons $T^{\prime}=Z \times_{Y} X$ et notons $\left(T_{\lambda}\right)_{\lambda=1, \ldots, n}$ (resp. $\left.\left(T_{\lambda}^{\prime}\right)_{\lambda=1, \ldots, n}\right)$ les composantes irréductibles de $T$ (resp. $T^{\prime}$ ), de telle manière que pour tout $\lambda$, $\left(T_{\lambda}^{\prime}\right)_{\text {red }}=T_{\lambda}$. Pour tout $\lambda$, on note $r_{\lambda}$ l'ordre de l'épaississement exact $T_{\lambda} \rightarrow T_{\lambda}^{\prime}$. On note enfin $g_{\lambda}: T_{\lambda} \rightarrow Z$ la restriction de $g$ à $T_{\lambda}$.

On peut alors factoriser le morphisme $N_{g} f$ comme suit:

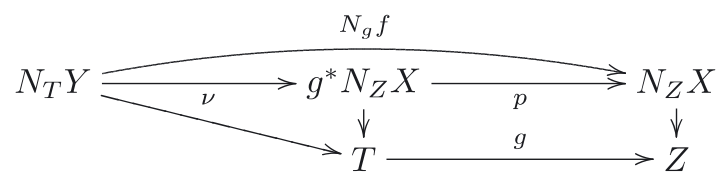

où le morphisme $v$ se décompose en la somme de morphismes $v_{\lambda}: N_{T_{\lambda}} Y \rightarrow g_{\lambda}^{*} N_{Z} X$ pour tout entier $\lambda$ dans $[1, n]$.

Pour tout $\lambda$, notons $\mathcal{I}_{\lambda}$ l'idéal de $T_{\lambda}$ dans $Y$. On pose

$$
N_{T_{\lambda}}^{\left(r_{\lambda}\right)} Y=\operatorname{Spec}_{T_{\lambda}}\left(\bigoplus_{n \in \mathbb{N}} \mathcal{I}_{\lambda}^{n r_{\lambda}} / \mathcal{I}_{\lambda}^{n r_{\lambda}+1}\right)
$$


Comme $T_{\lambda}$ est régulièrement immergé dans $Y$ de codimension $1, N_{T_{\lambda}}^{\left(r_{\lambda}\right)} Y$ est un fibré vectoriel de rang 1 sur $T_{\lambda}$.

Revenant à la construction de 2.4.9, on peut décrire le morphisme $v_{\lambda}$ par la factorisation suivante:

$$
N_{T_{\lambda}} Y \stackrel{\nu_{\iota_{\lambda}}}{\longrightarrow} N_{T_{\lambda}}^{\left(r_{\lambda}\right)} \underset{i_{\lambda}}{\longrightarrow} g_{\lambda}^{*} N_{Z} X .
$$

dans laquelle le morphisme $i_{\lambda}$ est une immersion fermée et le morphisme $l_{\lambda}$ est induit par le morphisme de $\mathcal{O}_{T_{\lambda}}$-modules

$$
\bigoplus_{n \in \mathbb{N}} \mathcal{I}_{\lambda}^{n r_{\lambda}} / \mathcal{I}_{\lambda}^{n r_{\lambda}+1} \rightarrow \bigoplus_{m \in \mathbb{N}} \mathcal{I}_{\lambda}^{m} / \mathcal{I}_{\lambda}^{m+1}
$$

Nous sommes donc dans les conditions d'application de la proposition 2.2.16 pour le morphisme $l_{\lambda}$. Comme par ailleurs, le morphisme $i_{\lambda}$ est un isomorphisme de fibrés vectoriels (car les deux fibrés sont de rang 1), on déduit de cette proposition que la partie (2) du diagramme suivant est commutative:

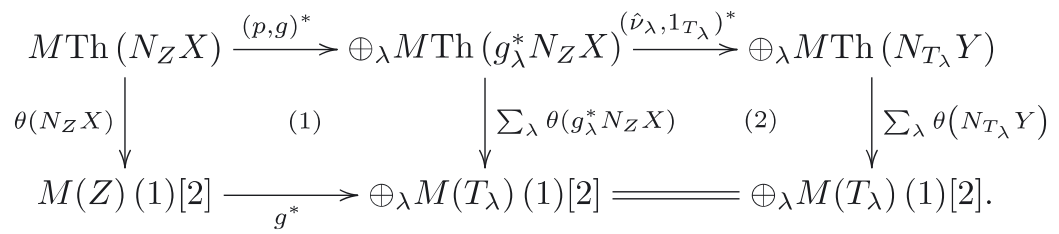

Comme la partie (1) est commutative d'après le troisième cas du lemme 2.2.15, on peut conclure.

2.6 - Propriétés supplémentaires

2.6.1 - Structure monoïdale et triangle de Gysin

Proposition 2.6.1. Soient $(X, Z)$ une paire fermée lisse de codimension n et $Y$ un schéma lisse. Notons $i: Z \rightarrow X$ l'immersion fermée associée $(X, Z)$ et posons $U=X-Z$.

Alors, le diagramme suivant est commutatif

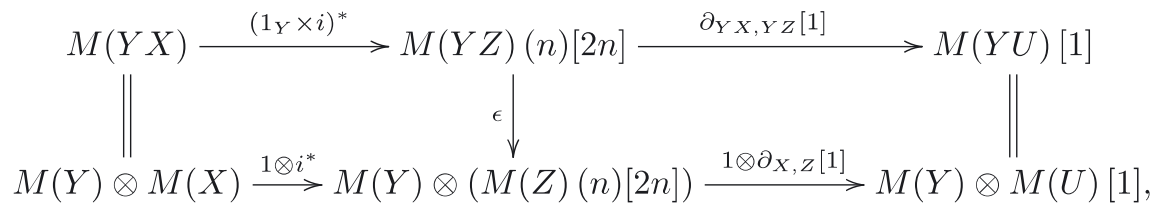


ayant noté \& l'isomorphisme de permutation

$$
Z(n)[2 n] \otimes M(Y) \otimes M(Z) \simeq M(Y) \otimes Z(n)[2 n] \otimes M(Z .)
$$

Remarque 2.6.2. Nous avons dégagé l'isomorphisme $\varepsilon$ dans cette proposition car celui-ci explique le signe dans la relation R3e des motifs génériques (cf. lemme 5.4.8 dans la deuxième partie).

PREuve: Remarquons tout d'abord que, puisque le foncteur $\mathrm{L}[Y] \otimes^{\operatorname{tr}}($.) est exact à droite, on obtient un isomorphisme canonique noté $\sigma$ dans le diagramme suivant de $\mathscr{N}_{k}^{\text {tr }}$, dont les lignes sont des suites exactes courtes:

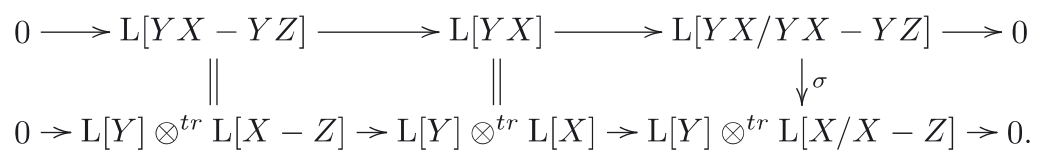

Puisque le morphisme de projection $Y X \rightarrow X$ est plat, on obtient des isomorphismes canoniques $D_{Y Z}(Y X) \simeq Y\left(D_{Z} X\right)$ et $N_{Y Z}(Y X) \simeq Y\left(N_{Z} X\right)$ qui sont compatibles aux morphismes de déformation au cône normal. Ainsi, on obtient le diagramme commutatif suivant, dont tous les morphismes sont des isomorphismes:

$$
\begin{array}{ccc}
M(Y X, Y Z) \longrightarrow & \sigma(Y) \otimes M(X, Z) \\
& \downarrow\left(1_{Y} \times d_{Z} X, s_{1}\right)_{*} \\
\left(d_{Y Z}(Y X), s_{1}\right)_{*} \downarrow & & \\
M\left(Y\left(D_{Z} X\right), Y\left(\mathbb{A}_{Z}^{1}\right)\right) \stackrel{\sigma}{\longrightarrow} & M(Y) \otimes M\left(D_{Z} X, \mathbb{A}_{Z}^{1}\right) \\
\left(d_{Y Z}^{\prime}(Y X), s_{0}\right)_{*} \uparrow & \uparrow\left(1_{Y} \times d_{Z}^{\prime} X, s_{0}\right)_{*} \\
M \operatorname{Th}\left(Y\left(N_{Z} X\right)\right) \stackrel{\sigma}{ } \longrightarrow & M(Y) \otimes M \operatorname{Th}\left(N_{Z} X\right) .
\end{array}
$$

On a noté $\sigma$ pour tous les isomorphismes obtenus comme indiqué en début de preuve (qui sont tous compatibles). Compte tenu du diagramme (D), il nous suffit donc de montrer que le morphisme $\sigma$ de la dernière ligne est égal, à travers les isomorphismes canoniques des motifs de Thom, au morphisme $\varepsilon$. D'après la définition de ces isomorphismes (cf.2.2.14), cela résulte du lemme:

Lemme 2.6.3. Soient $Z$ et $Y$ des schémas lisses, et $E$ un fibrévectoriel sur $Z$. Alors, pour tout entier naturel $r$, le diagramme suivant est commutatif:

$$
\begin{array}{cc}
M(\mathbb{P}(Y E)) \stackrel{\mathfrak{l}_{r}(Y E)}{\longrightarrow} & M(Y Z)(n)[2 n] \\
\| & \sim \downarrow \epsilon \\
M(Y) \otimes M(\mathbb{P}(E)) \stackrel{1_{Y} \otimes \mathfrak{l}_{r}(E)}{\longrightarrow} M(Y) \otimes(M(Z)(n)[2 n]) .
\end{array}
$$

Or ce lemme est un simple corollaire de 2.2.8. 
2.6.2 - Action des unités et résidu (spécialisation)

Au début de la preuve de la proposition 2.2.4, nous avons construit un isomorphisme canonique

$$
Z\{1\} \simeq M\left(\mathrm{G}_{m} /\{1\}\right)
$$

Autrement dit, si $i:\{1\} \rightarrow \mathbb{G}_{m}$ désigne l'immersion canonique, on dispose d’un triangle distingué canonique

$$
\mathrm{Z} \stackrel{i_{*}}{\longrightarrow} M\left(\mathrm{G}_{m}\right) \stackrel{\rho}{\longrightarrow} \mathrm{Z}\{1\} \stackrel{+1}{\longrightarrow} .
$$

Mais par ailleurs, le triangle de Gysin associé à la paire fermée $\left(\mathrm{A}_{k}^{1},\{0\}\right)$ s'écrit

$$
\mathrm{Z}\{1\} \stackrel{\partial}{\longrightarrow} M\left(\mathrm{G}_{m}\right) \stackrel{j_{*}}{\longrightarrow} M\left(\mathrm{~A}^{1}\right) \stackrel{+1}{\longrightarrow},
$$

où $j: \mathbb{G}_{m} \rightarrow \mathrm{A}_{k}^{1}$ désigne l'immersion ouverte canonique. Soit $p: \mathrm{A}_{k}^{1} \rightarrow$ $\rightarrow \operatorname{Spec}(k)$ la projection canonique. Comme le morphisme $(i p)_{*}$ est une section de $j_{*}$, on en déduit que le morphisme $\partial$ est une section de $\rho$.

Soit $X$ un schéma lisse. A l'aide de ces notations, l'isomorphisme de la proposition 2.2.4 est donné par la formule suivante

$$
\operatorname{Hom}\left(X, \mathrm{G}_{m}\right) \rightarrow \operatorname{Hom}_{D M_{-}^{e f f}(k)}(M(X), Z\{1\}), x \mapsto x_{*} \circ \rho .
$$

DÉfinition 2.6.4. Soit $X$ un schéma lisse.

Pour toute unité $x: X \rightarrow \mathrm{G}_{m}$, on pose avec les notations qui précèdent et celles de la définition 1.2.1: $\gamma_{x}=\left(x_{*} \circ \rho\right) \bigotimes 1_{X}: M(X) \rightarrow M(X)\{1\}$.

Dans la deuxième partie, nous aurons à considérer ce cup-produit avec une unité.

Proposition 2.6.5. Soit $(X, Z)$ une paire fermée lisse de codimension 1. On note $i: Z \rightarrow X$ l'immersion fermée et $j: X-Z \rightarrow X$ l'immersion ouverte canonique.

Supposons qu'il existe une fonction régulière $\pi: X \rightarrow \mathrm{A}_{k}^{1}$ telle que $Z$ est défini par l'équation $\{\pi=0\}$. On en déduit une unité de $X-Z$, encore notée $\pi: X-Z \rightarrow \mathrm{G}_{m}$. Alors, le diagramme suivant est commutatif:

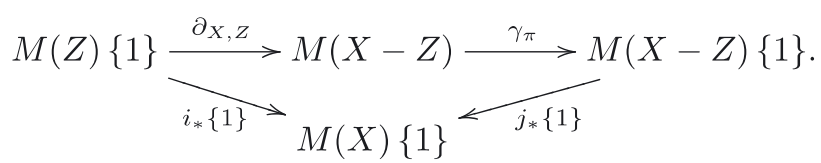


PREUve: On doit démontrer que le diagramme suivant est commutatif:

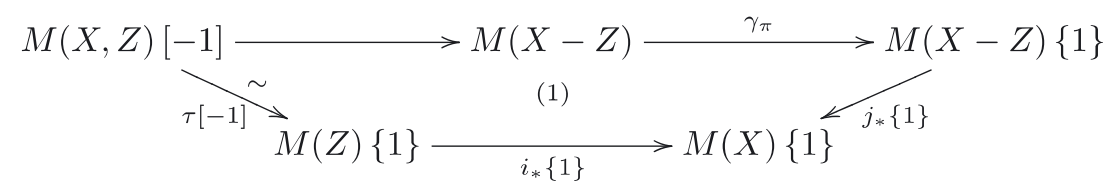

où $\tau$ est l'isomorphisme de pureté associé à $(X, Z)$ (cf. 2.2.5).

Or, puisque $\pi$ est une paramétrisation de $Z$, elle se relève en une fonction régulière $\pi: D_{Z} X \rightarrow \mathrm{A}_{k}^{1}$, qui est inversible sur $D_{Z} X-\mathrm{A}_{Z}^{1}$. Dès lors, le diagramme ci-dessus se déforme à travers le morphisme $d_{Z} X: X \rightarrow D_{Z} X$ en

$$
\begin{aligned}
& M\left(D_{Z} X, \mathbb{A}_{Z}^{1}\right)[-1] \longrightarrow M\left(D_{Z} X-\mathbb{A}_{Z}^{1}\right) \stackrel{\gamma_{\pi}}{\longrightarrow} M\left(D_{Z} X-\mathbb{A}_{Z}^{1}\right)\{1\} \\
& \widetilde{\sim} \sim \\
& M\left(\mathbb{A}_{Z}^{1}\right)\{1\} \underset{i_{*}\{1\}}{\longrightarrow} M\left(D_{Z} X\right)\{1\}
\end{aligned}
$$

où l'on a noté par abus $i$ et $j$ les immersions canoniques associées à la paire fermée $\left(D_{Z} X, \mathrm{~A}_{Z}^{1}\right)$. L'isomorphisme $\tau^{\prime}$ est obtenu par la composée des isomorphismes

$$
\begin{aligned}
M\left(D_{Z} X, \mathrm{~A}_{Z}^{1}\right) \stackrel{\left(d_{Z}^{\prime} X, s_{0}\right)_{*}^{-1}}{\longrightarrow} M \operatorname{Th}\left(N_{Z} X\right) \stackrel{\theta\left(N_{Z} X\right)}{\longrightarrow} M(Z)(1)[2] \\
\stackrel{\left(s_{1}\right)_{*}(1)[2]}{\longrightarrow} M\left(\mathrm{~A}_{Z}^{1}\right)(1)[2]
\end{aligned}
$$

où $s_{1}$ désigne l'immersion fermée unité de $\mathrm{A}_{Z}^{1}$.

Or le morphisme $\left(d_{Z} X\right)_{*}: M(X) \rightarrow M\left(D_{Z} X\right)$ est scindé. Si l'on considère la projection canonique $p: D_{X} \rightarrow \mathrm{A}_{X}^{1}$, cela résulte du diagramme commutatif ci-dessous, puisque $\left(s_{1}\right)_{*}$ est un isomorphisme:

$$
M(X) \underset{\left(s_{1}\right)_{*}}{\underset{\longrightarrow}{\longrightarrow} M\left(\mathbb{A}_{X}^{1}\right){ }_{p_{*}}} M\left(D_{Z} X\right) .
$$

On en déduit que la commutativité du diagramme (1) est équivalente à celle du diagramme (2). Or le diagramme (2) est aussi la déformation de

$$
\begin{aligned}
& M \operatorname{Th}\left(N_{Z} X\right)[-1] \longrightarrow M\left(N_{Z} X-Z\right) \stackrel{\gamma_{\pi}}{\longrightarrow} M\left(N_{Z} X-Z\right)\{1\} \\
& \theta\left(N_{Z} X\right)[-1] \sim \\
& M(Z)\{1\} \longrightarrow
\end{aligned}
$$


où $i$ et $j$ désignent par un nouvel abus les immersions canoniques associées à la paire fermée $\left(N_{Z} X, Z\right)$, et $\pi: N_{Z} X \rightarrow \mathrm{A}_{k}^{1}$ la fonction régulière induite par $\pi$. Notons que l'on a utilisé le fait que, dans la catégorie $D M_{-}^{e f f}(k)$, $\left(s_{0}\right)_{*}=\left(s_{1}\right)_{*}$ où $s_{0}$ et $s_{1}$ désignent respectivement la section nulle et la section unité de $A_{Z}^{1}$.

Ainsi, du fait que le morphisme $\left(d_{Z}^{\prime} X, s_{0}\right)_{*}$ est un isomorphisme, la commutativité du diagramme (3) implique la commutativité du diagramme (2).

Soit $p: N_{Z} X \rightarrow Z$ le morphisme de projection canonique. Puisque $\pi$ est une paramétrisation de $Z$, le morphisme $\pi \times p: N_{Z} X \rightarrow \mathbb{A}_{Z}^{1}$ est un isomorphisme. Celui-ci induit un isomorphisme de paires fermées $\left(N_{Z} X, Z\right) \rightarrow\left(A_{Z}^{1}, Z\right)$. Par ailleurs, par définition, l'isomorphisme $\pi \times p$ envoie la fonction régulière $\pi: N_{Z} X \rightarrow \mathrm{A}_{k}^{1}$ sur la fonction régulière triviale $\tau: A_{Z}^{1} \rightarrow A_{k}^{1}$. Il en résulte que la commutativité du diagramme (3) est finalement équivalente à la commutativité de

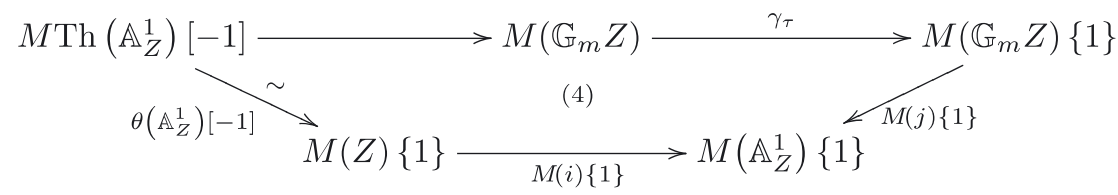

où, par un dernier abus, $i$ désigne la section nulle de $\mathrm{A}_{Z}^{1}$, et $j$ l'immersion ouverte complémentaire. Pour la compatibilité des isomorphismes des espaces de Thom, on a utilisé la première assertion du lemme 2.2.15.

Or, $\theta\left(\mathbb{A}_{Z}^{1}\right)=\theta\left(\mathbb{A}_{k}^{1}\right) \times 1_{Z}=1$, et d'après la proposition 2.6.1,

$$
\partial_{\left(\mathrm{A}_{Z}^{1}, Z\right)}=\partial_{\left(\mathrm{A}_{k}^{1},\{0\}\right)} \otimes 1_{Z}
$$

D’après l'étude qui précède la définition 2.6.4, le morphisme résidu associé à la paire fermée $\left(A_{k}^{1},\{0\}\right)$ est une section de l'épimorphisme $\rho: M\left(\mathrm{G}_{m}\right) \rightarrow Z\{1\}$. Donc la commutativité du diagramme (4) se réduit à la commutativité du diagramme

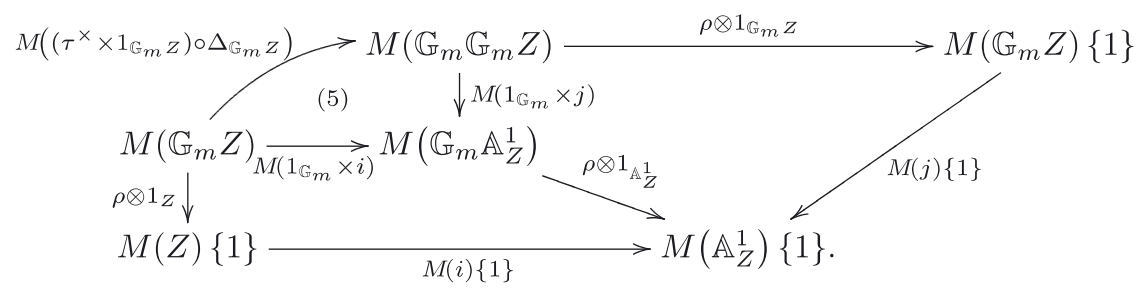

Le problème est donc réduit à la commutativité de la partie (5) de ce diagramme, qui est évidente (et déjà vraie dans la catégorie des schémas). 


\section{Catégorie des motifs génériques}

\section{Définition}

\section{1 - Pro-objets}

Soit $\mathscr{T}$ une catégorie. Rappelons qu'un pro-objet de $\mathscr{T}$ est un foncteur $\mathcal{I} \rightarrow \mathscr{T}, X \mapsto X_{i}$ pour $\mathcal{I}$ une catégorie cofiltrante essentiellement petite.

Nous utiliserons la notation suggestive

$$
X_{\bullet}=\lim _{i \in \mathcal{I}}^{\sim} X_{i}
$$

pour noter les pro-objets. Cette notation est justifiée puisque $X$ est la limite projective dans la catégorie des pro-objets des $X_{i}$ vus comme proobjets constants. Le tilde permet de différencier cette limite projective formelle de la limite projective calculée dans $\mathscr{T}$.

Un morphisme de pro-objets $\lim _{i \in \mathcal{I}}^{\sim} X_{i} \rightarrow \lim _{j \in \mathcal{J}}^{\sim} Y_{j}$ est la donnée d'un foncteur $F: \mathcal{J} \rightarrow \mathcal{I}$ et une transformation naturelle $X_{F(\bullet)} \rightarrow Y_{\bullet}$. Ces morphismes sont soumis à une relation d'équivalence qui fait que

$$
\operatorname{Hom}_{\text {pro- } \mathscr{T}}\left(X_{\bullet}, Y_{\bullet}\right)=\lim _{j \in \mathcal{J}} \lim _{i \in \mathcal{T}^{o p}} \operatorname{Hom}_{\mathscr{T}}\left(X_{i}, Y_{j}\right)
$$

On note pro- $\mathscr{T}$ la catégorie des pro-objets de $\mathscr{T}$.

Lorsque $\mathscr{T}$ est abélienne, pro- $\mathscr{T}$ est naturellement une catégorie abélienne.

Par contre dans le cas où $\mathscr{T}$ est triangulée, pro- $\mathscr{T}$ n'est en général pas triangulée à moins que $\mathscr{T}$ soit semi-simple.

La catégorie pro- $\mathscr{T}$ est seulement additive, munie d'un endofoncteur induit par la suspension de $\mathscr{T}$. Si $X_{\bullet} \rightarrow Y_{\bullet} \rightarrow Z_{\bullet} \rightarrow X_{\bullet}[1]$ est une suite de morphismes de pro- $\mathscr{T}$, nous dirons que c'est un triangle pro-distingué s'il est isomorphe à la limite projective formelle d'un système filtrant de triangles distingués de $\mathscr{T}$.

Notons enfin que si $\mathscr{T}$ est une catégorie symétrique monoïdale, il existe une unique structure symétrique monoïdale sur pro- $\mathscr{T}$ dont le produit tensoriel commute aux limites projectives formelles.

\section{2 - Algèbres essentiellement lisses et modèles}

Rappelons la définition classique suivante: 
DÉfInITION 3.2.1. Soit $\mathcal{O}$ une $k$-algèbre. Nous dirons que $\mathcal{O}$ est essentiellement de type fini (resp. essentiellement lisse) sur $k$ si il existe une $k$ algèbre $A$ de type fini (resp. lisse), un idéal premier $x$ de $A$ et un isomorphisme $\mathcal{O} \simeq A_{x}$.

Remarque 3.2.2. Puisque $k$ est parfait, une $k$-algèbre est essentiellement lisse si et seulement si elle est régulière et essentiellement de type fini.

EXEMPLE 3.2.3. Les $k$-algèbres essentiellement lisses de dimension 0 sont les corps de fonctions. Les $k$-algèbres essentiellement lisses de dimension 1 sont les anneaux de valuation discrète essentiellement de type fini sur $k$. Si $E$ est un corps de fonctions, et $v$ une valuation sur $E$, rappelons que $v$ est dite géométrique si elle est de rang 1 et son anneau de valuation est essentiellement de type fini sur $k$. Ceci implique que $v$ est une valuation discrète.

Plus généralement les anneaux essentiellement lisses correspondent aux anneaux locaux des schémas lisses. Dans cet esprit, nous adoptons la définition suivante:

DÉFINITION 3.2.4. Soit $\mathcal{O}$ une $k$-algèbre essentiellement lisse.

Un modèle de $\mathcal{O}$ est un couple $(X, x)$ formé d'un schéma lisse $X$ et d'un morphisme $x: \operatorname{Spec}(\mathcal{O}) \rightarrow X$ tel que, si l'on confond $x$ avec l'image du point fermé de $\operatorname{Spec}(\mathcal{O})$ dans $X$, le morphisme induit $x^{\sharp}: \mathcal{O}_{X, x} \rightarrow \mathcal{O}$ est un isomorphisme.

On note $\mathcal{M}^{\text {lis }}(\mathcal{O} / k)$ l'ensemble ordonné par inclusion des sous- $k$-algèbres $A \subset \mathcal{O}$ telles que $\operatorname{Spec}(A)$, muni du $\mathcal{O}$-point évident, est un modèle de $\mathcal{O}$.

Puisque $k$ est supposé parfait, l'ensemble $\mathcal{M}^{l i s}(\mathcal{O} / k)$ est non vide et filtrant pour l'ordre induit par l'inclusion.

DÉFINITION 3.2.5. On considère les pro-objets de schémas lisses suivants: schéma

(1) Soit $\mathcal{O}$ une $k$-algèbre essentiellement lisse. On lui associe un pro-

$$
(\mathcal{O})=\lim _{A \in \mathcal{M}^{l i s\left(\mathcal{O} / k^{o p}\right)}} \operatorname{Spec}(A .)
$$

(2) Si $X$ est un schéma lisse, et $x$ un point de $X$, on définit le localisé 
de $X$ en $x$ comme le pro-schéma

$$
X_{x}=\lim _{U \subset \overleftarrow{X, x}, \tilde{U} U} U
$$

où $U$ parcourt l'ensemble ordonné cofiltrant des voisinages ouverts de $x$ dans $X$.

Si $F$ est un préfaisceau sur $\mathscr{L}_{k}$, et $\mathcal{X}=\left(X_{i}\right)_{i \in \mathcal{I}}$ un pro-objet de schémas lisses, on pose

$$
\hat{F}(\mathcal{X})=\lim _{i \in \mathcal{I}^{o p}} F\left(X_{i}\right)
$$

3.2.6. Les pro-objets du type $(\mathcal{A})$ (resp. $X_{x}$ ) pour une $k$-algèbre essentiellement lisse $\mathcal{A}$ (resp. un schéma pointé lisse $(X, x)$ ) sont essentiellements affines sur $k$ au sens de [EGA4, 8.13.4]. De plus $\operatorname{Spec}(\mathcal{A})$ (resp. $\left.\operatorname{Spec}\left(\mathcal{O}_{X, x}\right)\right)$ est la limite projective de $(\mathcal{A})\left(\operatorname{resp} . X_{x}\right)$. On déduit donc de [EGA4, 8.13.2] les assertions suivantes:

(1) Soit $\mathcal{A}$ et $\mathcal{B}$ deux $k$-algèbres essentiellement lisses. Alors il existe un isomorphisme canonique $\operatorname{Hom}_{\text {pro- } \mathscr{L}_{k}}((\mathcal{B}),(\mathcal{A})) \simeq \operatorname{Hom}_{k}(\mathcal{A}, \mathcal{B})$

(2) Soit $\mathcal{O}$ une $k$-algèbre essentiellement lisse et $(X, x)$ un modèle de $\mathcal{O}$. Alors le morphisme $x$ induit un isomorphisme canonique $(\mathcal{O}) \rightarrow X_{x}$.

Remarque 3.2.7. Si $\mathcal{O}$ est une $k$-algèbre essentiellement lisse, on dit suivant une terminologie courante que le foncteur $F \mapsto \hat{F}(\mathcal{O})$ est pro-représenté par $(\mathcal{O})$ - vu comme pro-objet de faisceaux représentables. D'après la deuxième propriété ci-dessus, c'est un foncteur fibre pour la topologie de Zariski sur $\mathscr{L}_{k}$. Par ailleurs, puisque pour tout schéma lisse $X$, et tout point $x \in X$, la $k$-algèbre $\mathcal{O}_{X, x}$ est essentiellement lisse, la famille formée par les foncteurs fibres associés aux $k$-algèbres essentiellement lisses est conservative pour la topologie de Zariski. Suivant [SGA4], on appelle morphisme de spécialisation toute transformation naturelle entre deux foncteurs fibres; cela revient à considérer les morphismes des pro-objets qui les proreprésentent.

\section{3 - Définition et propriété fondamentale}

DÉfinition 3.3.1. Soient $E$ un corps de fonctions et $n \in Z$ un entier. On définit le motif générique de $E$ en poids n, noté $M_{g m}(E)\{n\}$, comme 
le pro-objet de $D M_{g m}(k)$

$$
M_{g m}(E)\{n\}=\lim _{A \in \mathcal{M}^{\leftarrow i}(E / k)^{o p}} M_{g m}(\operatorname{Spec}(A))\{n\} .
$$

Si $n \geq 0$, on dit que ce motif générique est effectif.

On note $D M_{g m}^{(0)}(k)$ (resp. $D M_{g m}^{e f f,(0)}(k)$ ) la sous-catégorie de pro-D $M_{g m}(k)$ (resp. de pro-D $M_{g m}^{\text {eff }}(k)$ ) formée des objets $M_{g m}(E)\{n\}$ où $E$ est un corps de fonctions, et $n$ un entier relatif (resp. un entier naturel). On l'appelle la catégorie des motifs génériques (resp. motifs génériques effectifs).

D'après le théorème de simplification de Voevodsky [Voe02], 4.10, le foncteur canonique

$$
D M_{g m}^{e f f,(0)}(k) \rightarrow D M_{g m}^{(0)}(k)
$$

est pleinement fidèle.

\section{4 - Liens avec la t-structure homotopique}

Cette sous-section vise à éclaircir l'interprétation des motifs génériques en tant que points pour les faisceaux homotopiques.

Si $\mathcal{M}$ est un complexe motivique, on note $\underline{\mathrm{H}}^{p}(\mathcal{M})$ son $p$-ième groupe de cohomologie calculé dans la catégorie des faisceaux avec transferts. Rappelons que par définition, ce faisceau est invariant par homotopie. La catégorie $D M_{-}^{\text {eff }}(k)$ porte ainsi une t-structure, dite homotopique, telle que le foncteur

$$
\underline{\mathrm{H}}^{0}: D M_{-}^{e f f}(k) \rightarrow \mathrm{H} \mathscr{N}_{k}^{\mathrm{tr}}
$$

est le foncteur cohomologique associé. La catégorie $\mathrm{H} / N_{k}^{\mathrm{tr}}$ est le coeur de $D M_{-}^{e f f}(k)$ pour la t-structure homotopique.

Proposition 3.4.1. Le foncteur

$$
\begin{aligned}
\mathrm{H} N_{k}^{\mathrm{tr}} & \rightarrow \mathscr{A b} \mathscr{\mathscr { C }}_{k} \\
F & \mapsto(E / k \mapsto \hat{F}(E))
\end{aligned}
$$

est exact, conservatif et commute aux limites inductives.

Preuve: Soit $E$ un corps de fonctions. Puisque $E$ est un anneau local hensélien, le pro-objet $(E)$ pro-représente en fait un foncteur fibre pour la 
topologie de Nisnevich. L'assertion sur l'exactitude et la commutation aux limites inductives résulte du fait que le foncteur d'oubli de $\mathrm{H} / N_{k}^{\mathrm{tr}}$ dans la catégorie des faisceaux Nisnevich est exact et commute aux limites inductives (cf. [Dég07, 4.4.15]). La conservativité résulte de [Dég07, 4.4.8].

Suivant Voevodsky, on introduit la construction suivante:

DÉfINITION 3.4.2. Soient $F$ un faisceau homotopique et $X$ un schéma lisse. On définit le groupe abélien $F_{-1}(X)$ par la suite exacte courte

$$
0 \rightarrow F\left(\mathrm{~A}^{1} \times X\right) \stackrel{F(j)}{\longrightarrow} F\left(\mathrm{G}_{m} \times X\right) \rightarrow F_{-1}(X) \rightarrow 0
$$

où $j: \mathrm{G}_{m} \times X \rightarrow \mathrm{A}^{1} \times X$ désigne l'immersion ouverte canonique.

On a ainsi associé à $F$ un faisceau $F_{-1}$ pour la topologie de Nisnevich sur $\mathscr{L}_{k}$ puisque la suite exacte courte qui intervient dans la définition ci-dessus est scindée. Le préfaisceau $F_{-1}$ est un faisceau pour la topologie de Nisnevich. Il est bien sûr invariant par homotopie et muni de transferts. On obtient donc un endofoncteur de $\mathrm{H} \mathscr{N}_{k}^{\text {tr }}$ qui possède la propriété remarquable suivante:

\section{Proposition 3.4.3. L'endofoncteur}

$$
\mathrm{H} \mathscr{N}_{k}^{\mathrm{tr}} \rightarrow \mathrm{H} \mathcal{N}_{k}^{\mathrm{tr}}, F \mapsto F_{-1}
$$

est exact.

Preuve: On fixe une suite exacte $0 \rightarrow F \rightarrow G \rightarrow H \rightarrow 0$ de faisceaux homotopiques et on montre que la suite $0 \rightarrow F_{-1} \rightarrow G_{-1} \rightarrow H_{-1} \rightarrow 0$ est encore exacte.

Puis, d'après la proposition 3.4.1, ayant fixé un corps de fonctions $E$, il suffit de montrer que la suite de groupes abéliens

$$
0 \rightarrow F_{-1}(E) \rightarrow G_{-1}(E) \rightarrow H_{-1}(E) \rightarrow 0
$$

est finie.

Par définitition, cette suite est facteur directe de la suite courte

$$
0 \rightarrow F\left(\mathrm{G}_{m} \times(E)\right) \rightarrow G\left(\mathrm{G}_{m} \times(E)\right) \rightarrow H\left(\mathrm{G}_{m} \times(E)\right) \rightarrow 0,
$$

obtenue comme limite inductive filtrante suivant $\mathcal{M}^{l i s}\left(E / k^{o p}\right)$. Cette suite est exacte, puisque d'après [Dég07, 4.4.10], $\mathrm{H}^{1}\left(\mathrm{G}_{m} \times(E) ; F\right)=0$. 
On rappelle que d'après [Voe00b, 3.2.6], il existe un morphisme pleinement fidèle canonique

$$
\imath: D M_{g m}^{e f f}(k) \rightarrow D M_{-}^{e f f}(k) .
$$

Ceci nous permet de voir par abus les motifs géométriques comme des complexes motiviques. De plus pour tout couple $(A, B)$ d'objets de $D M_{-}^{\text {eff }}(k)$ tel que $A$ appartienne à l'image essentielle de $l$, la catégorie $D M_{-}^{\text {eff }}(k)$ possède un hom interne (cf. [Voe00b, 3.2.8]) évalué en $(A, B)$ que l'on note simplement $\underline{\operatorname{Hom}}(A, B)$.

Proposition 3.4.4. Soient $E$ un corps de fonctions, et $\mathcal{M}$ un complexe motivique.

Alors, pour tout entier $n \in \mathbb{N}$, il existe un isomorphisme canonique naturel en $\mathcal{M}$ :

$$
\operatorname{Hom}_{\text {pro- } D M_{-}^{e f f}(k)}\left(M_{g m}(E)\{n\}, \mathcal{M}\right) \simeq \underline{\mathrm{H}}^{0}(\mathcal{M})_{-n}(E) .
$$

PREUVE: Le cas $n=0$ est une simple application de la suite spectrale d'hypercohomologie

$$
E_{2}^{p, q}=\mathrm{H}^{p}\left(E ; \underline{\mathrm{H}}^{q}(\mathcal{M})\right) \Rightarrow \mathrm{H}^{p+q}(E ; \mathcal{M})
$$

obtenue par limite inductive filtrante des suites spectrales d'hypercohomologie associées aux $k$-algèbres $A \in \mathcal{M}^{\text {lis }}(\mathcal{E} / k)$.

Pour $n>0$, on en déduit donc

$$
\operatorname{Hom}\left(M_{g m}(E)\{p\}, \mathcal{M}\right)=\underline{\mathrm{H}}^{0}(\underline{\operatorname{Hom}}(Z\{\{p\}, \mathcal{M}))(E) .
$$

LeMme 3.4.5. Soit F un faisceau homotopique.

Alors, il existe un isomorphisme canonique $\underline{\operatorname{Hom}}(Z\{1\}, F) \simeq F_{-1}$.

Preuve: On commence par montrer que le complexe motivique $\underline{\operatorname{Hom}}(Z\{1\}, F)$ est concentré en degré 0 . Soit $p \in Z^{*}$. D’après la proposition 3.4.1, il s'agit de montrer que pour tout corps de fonctions $E$,

$$
\underline{\mathrm{H}}^{p}(\underline{\operatorname{Hom}}(Z\{1\}, F))(E)=0 .
$$

Or, d'après le premier point de cette preuve,

$$
\underline{\mathrm{H}}^{p}(\underline{\operatorname{Hom}}(Z\{1\}, F))(E)=\operatorname{Hom}\left(M_{g m}(E)\{1\}, F[p]\right) .
$$

Par ailleurs, $M_{g m}(E)\{1\}$ est facteur direct de $M_{g m}\left(\mathbb{G}_{m}\right) \otimes M_{g m}(E)$. Donc, $\operatorname{Hom}\left(M_{g m}(E)\{1\}, F[p]\right) \subset \operatorname{Hom}\left(M_{g m}\left(\mathbb{G}_{m}\right) \otimes M_{g m}(E), F[p]\right) \simeq \mathrm{H}^{p}\left(\mathbb{G}_{m} \times(E) ; F\right)$, 
où l'on a appliqué [Voe00b, 3.2.3] pour le dernier isomorphisme. Or ce dernier groupe est nul pour $p \neq 0$ d'après [Dég07, 4.4.10].

Considérons maintenant un schéma lisse $X$. Comme le foncteur $\operatorname{Hom}(., F)$ est triangulé, en l'appliquant au triangle distingué

$$
M_{g m}(X)\{1\} \rightarrow M_{g m}\left(\mathrm{G}_{m}\right) \otimes M_{g m}(X) \rightarrow M_{g m}\left(\mathrm{~A}_{k}^{1}\right) \otimes M_{g m}(X) \stackrel{+1}{\longrightarrow}
$$

on obtient par définition de $F_{-1}$ la suite exacte

$$
0 \rightarrow F_{-1}(X) \stackrel{\eta_{X}}{\longrightarrow} \Gamma(X ; \underline{\operatorname{Hom}}(Z\{1\}, F)) \rightarrow \mathrm{H}^{1}\left(\mathrm{~A}_{k}^{1} \times X ; F\right) .
$$

Il reste à montrer que le morphisme de faisceaux $\eta$ est un isomorphisme. Pour cela, il suffit par une nouvelle application de 3.4.1 de montrer que pour tout corps de fonctions $E$, le morphisme $\eta_{E}$ est un isomorphisme. On considère alors la limite inductive de la suite exacte ci-dessus pour $X=\operatorname{Spec}(A)$, où $A$ parcourt l'ensemble filtrant $\mathcal{M}^{l i s}(E / k)$ et on remarque que d'après le théorème fondamental de Voevodsky (cf. [Dég07, 5.1]), $\mathrm{H}^{1}\left(\mathrm{~A}_{k}^{1} \times(E) ; F\right)=0$.

Comme Z $\{1\}$ est concentré en degré 0 , le foncteur $\underline{\operatorname{Hom}}(Z\{1\},$.$) est t-$ exact à gauche pour la t-structure homotopique. Puisqu'il induit d'après le lemme précédent un foncteur exact sur le coeur homotopique de $D M_{-}^{\text {eff }}(k)$, on en déduit qu'il est même t-exact ce qui permet de conclure.

3.4.6. Si $E$ est un corps de fonctions et $n \in \mathbb{N}$ un entier naturel, le foncteur

$$
\mathrm{H} \mathcal{N}_{k}^{\mathrm{tr}} \rightarrow \mathscr{b} b, F \mapsto \mathrm{Hom}_{\text {pro-D }} M_{g m}^{\text {eff }(k)}\left(M_{g m}(E)\{n\}, F\right)=\hat{F}_{-n}(E)
$$

est donc exact. Il commute par ailleurs aux sommes directes quelconques et définit donc un "foncteur fibre" sur $\mathrm{H} \mathscr{N}_{k}^{\text {tr }}$ par extension de la terminologie de [SGA4] hors du cadre des topos.

On notera par ailleurs que la famille formée par ces points est conservative, en vertu de la proposition 3.4.1.

Corollaire 3.4.7. Le foncteur

$$
D M_{g m}^{e f f,(0)}(k) \rightarrow \operatorname{pro}-\mathrm{H} \mathcal{N}_{k}^{\mathrm{tr}}, M(E)\{n\} \mapsto \underline{\mathrm{H}}^{0}(M(E)\{n\})
$$

est pleinement fidèle. De plus, pour tout corps de fonctions $E$ et tout $(n, i) \in \mathbb{N} \times \mathbb{N}^{*}, \underline{H}^{i}(M(E)\{n\})=0$. 
Ainsi, la catégorie $D M_{g m}^{e f f}\left({ }^{(0)}(k)\right.$ est canoniquement une sous-catégorie pleine de la catégorie abélienne pro- $\mathrm{H} N_{k}^{\mathrm{tr}}$, bien qu'elle soit construite à partir d'une catégorie triangulée.

\section{Rappels sur les modules de cycles}

Dans [Ros96], Rost a introduit la notion de module de cycles de manière à pouvoir construire un complexe de Gersten analogue à celui obtenu par Kato avec la K-théorie de Milnor (cf. [Kat86]). Pour la commodité du lecteur, nous rappelons maintenant cette théorie dans le cas particulier où la base est le corps parfait $k$. Par convention, tous les pré-modules de cycles considérés seront des pré-modules de cycles sur $k$ et nous ne précisons donc plus la base.

\section{1 - Pré-modules de cycles}

On peut décrire les modules de cycles comme des foncteurs à valeur dans les groupes abéliens sur une catégorie (voir aussi [Dég06, 1.1]) que nous décrivons maintenant.

Nous noterons $\tilde{\mathscr{E}}_{k}$ la catégorie dont les objets sont les couples $(E, n)$ tel que $E$ est un corps de fonctions $n \in Z$ un entier. Les morphismes de $\tilde{\mathscr{C}}_{k}$ sont définis par les générateurs et relations suivants - ci-dessous, nous appelons corps valué tout corps de fonctions muni d'une valuation géométrique (voir les notations en début d'article):

\section{Générateurs:}

D1: $\varphi_{*}:(E, n) \rightarrow(L, n)$ pour $\varphi: E \rightarrow L, n \in Z$.

D2: $\varphi^{*}:(L, n) \rightarrow(E, n)$, pour $\varphi: E \rightarrow L$ fini, $n \in Z$.

D3: $\gamma_{x}:(E, n) \rightarrow(E, n+r)$, pour $x \in K_{r}^{M}(E), n \in Z$.

D4: $\partial_{v}:(E, n) \rightarrow(\kappa(v), n-1)$, pour $(E, v)$ corps valué, $n \in Z$.

\section{Relations:}

R0: Pour tous $x \in K_{*}^{M}(E), y \in K_{*}^{M}(E), \gamma_{x} \circ \gamma_{y}=\gamma_{x . y}$.

R1a: $(\psi \circ \varphi)_{*}=\psi_{*} \circ \varphi_{*}$.

R1b: $(\psi \circ \varphi)^{*}=\varphi^{*} \circ \psi^{*}$.

R1c: Soit $\varphi: K \rightarrow E, \psi: K \rightarrow L$ fini. Pour $z \in \operatorname{Spec}\left(E \otimes_{K} L\right)$, notons $\bar{\varphi}_{z}: L \rightarrow E \otimes_{K} L / z$ et $\bar{\psi}_{z}: E \rightarrow E \otimes_{K} L / z$ les morphismes induits:

$\psi_{*} \varphi^{*}=\sum_{z \in \operatorname{Spec}\left(E \otimes_{K} L\right)} \lg \left(E \otimes_{K} L_{z}\right) \cdot\left(\bar{\varphi}_{z}\right)^{*}\left(\bar{\psi}_{z}\right)_{*}$,

où pour un anneau artinien $A$, l'entier $\lg (A)$ désigne la longueur de $A$. 
R2a: Pour tous $\varphi: E \rightarrow L, x \in K_{*}^{M}(E), \varphi_{*} \circ \gamma_{x}=\gamma_{\varphi_{*}(x)} \circ \varphi_{*}$.

R2b: Pour tous $\varphi: E \rightarrow L$ fini, $x \in K_{*}^{M}(E), \varphi^{*} \circ \gamma_{\varphi_{*}(x)}=\gamma_{x} \circ \varphi^{*}$.

R2c: Pour tous $\varphi: E \rightarrow L$ fini, $y \in K_{*}^{M}(L), \varphi^{*} \circ \gamma_{y} \circ \varphi_{*}=\gamma_{\varphi^{*}(y)}$.

R3a: Soit $\varphi: E \rightarrow L, v$ valuation sur $L, w$ valuation sur $E$ telles que $\left.v\right|_{E^{\times}}=e . w$ avec $e>0$. Notons $\bar{\varphi}: \kappa(w) \rightarrow \kappa(v)$ le morphisme induit:

$$
\partial_{v} \circ \varphi_{*}=e \cdot \bar{\varphi}_{*} \circ \partial_{w} .
$$

R3b: Soit $\varphi: E \rightarrow L$ fini, $v$ valuation $\operatorname{sur} E$. Si $w$ est une extension de $v$ à $L$, on note $\bar{\varphi}_{w}$ le morphisme induit par $\varphi$ sur les corps résiduels:

$$
\partial_{v} \circ \varphi^{*}=\sum_{w / v} \bar{\varphi}_{w}^{*} \circ \partial_{w} .
$$

R3c: Soit $\varphi: E \rightarrow L, v$ valuation sur $L$ nulle $\operatorname{sur} E^{\times}: \partial_{v} \circ \varphi_{*}=0$.

R3d: Pour $(E, v)$ corps valué, $\pi$ uniformisante de $v: \partial_{v} \circ \gamma_{\{-\pi\}} \circ \varphi_{*}=\bar{\varphi}_{*}$.

R3e: Pour $(E, v)$ corps valué, $u$ unité de $v: \partial_{v} \circ \gamma_{\{u\}}=-\gamma_{\{\bar{u}\}} \circ \partial_{v}$.

La définition suivante est trivialement équivalente à celle de [Ros96, (1.1)]:

DÉFINITION 4.1.1. Un pré-module de cycles est un foncteur $M: \tilde{\mathscr{E}}_{k} \rightarrow \mathscr{b b}$. Un morphisme de pré-modules de cycles est une transformation naturelle.

\section{2 - Modules de cycles}

Dans le but de construire, à l'aide d'un pré-module de cycles, un complexe du type complexe de Gersten, nous définissons suivant Rost les différentielles de ce complexe. La théorie de Rost nécessite de considérer des $k$-schémas qui ne sont pas nécessairement de type fini. Donc, jusqu'à la fin de ces rappels, nous changeons la convention de l'article: tous les schémas considérés sont supposés munis d'une structure de $k$-schéma essentiellement de type fini.

DÉfinition 4.2.1. Soient $M$ un pré-module de cycles, et $X$ un schéma.

(1) Pour tout point $x$ de $X$, on pose $M(x)=M(\kappa(x))$.

(2) Supposons $X$ normal. Soit $\eta$ son point générique et $z$ un point de codimension 1 . Alors $z$ correspond à une valuation discrète $v_{z}$ sur le corps des fonctions $\kappa(\eta)$ de $X$. D'après l'axiome $D 4$, on définit donc

$$
\partial_{z}^{X}=\partial_{v_{z}}: M(\eta) \rightarrow M(z) .
$$


(3) Soient $x$ et $y$ des points de $X$. On note $Z$ l'adhérence réduite de $x$ dans $X$. On note $\tilde{Z}$ le normalisé de $Z$; le morphisme canonique $f: \tilde{Z} \rightarrow Z$ est fini.

Supposons que $y \in Z^{(1)}$, et notons $\tilde{Z}_{y}$ la fibre de $f$ au-dessus de $y$. Alors, tout point $z \in \tilde{Z}_{y}$ est de codimension 1 dans $\tilde{Z}$. Pour un tel point $z$, on note $\varphi_{z}: \kappa(y) \rightarrow \kappa(z)$ le morphisme induit par $f$ sur les corps résiduels; c'est un morphisme fini.

On pose alors:

$$
\partial_{y}^{x}=\left\{\begin{array}{ll}
\sum_{z \in \tilde{Z}_{y}} \varphi_{z}^{*} \circ \partial_{z}^{\tilde{Z}} & \text { si } y \in Z^{(1)} \\
0 & \text { sinon }
\end{array}\right\}: M(x) \rightarrow M(y) .
$$

Avec ces notations, nous pouvons introduire les deux axiomes suivants sur les pré-modules de cycles:

DÉfinition 4.2.2. Soit $M$ un pré-module de cycles. On dit que $M$ est un module de cycles si et seulement si les conditions suivantes sont vérifiées:

(FD) Pour tout schéma normal $X$ de point générique $\eta$, et pour tout $\rho \in M(\eta)$, l'ensemble $\left\{x \in X^{(1)} \mid \partial_{x}^{\eta}(\rho) \neq 0\right\}$ est fini.

(C) Pour tout schéma $X$ intègre, local et de dimension 2, dont on note $\eta$ le point générique et $x_{0}$ le point fermé, on a

$$
\sum_{x \in X^{(1)}} \partial_{x_{0}}^{x} \circ \partial_{x}^{\eta}=0 .
$$

Comme annoncé, on obtient finalement le complexe de (co)cycles à coefficients dans $M$ :

DÉfinition 4.2.3. Soient $M$ un module de cycles, et $X$ un schéma.

Pour tout entier $p \in \mathbb{N}$, on pose:

$$
C^{p}(X ; M)=\bigoplus_{x \in X^{(p)}} M(x) .
$$

Pour tout entier $p \in \mathbb{N}$, on définit un morphisme

$$
d_{X, M}^{p}=\sum_{(x, y) \in X^{(p)} \times X^{(p+1)}} \partial_{y}^{x} .
$$

D'après les axiomes des modules de cycles, $C^{*}(X ; M)$, muni de ces morphismes, est un complexe. On définit le $p$-ième groupe de Chow à coefficients dans $M$, noté $A^{p}(X ; M)$, comme le $p$-ième groupe de cohomologie du complexe de cocycles à coefficients dans $M$. 
Notons que pour tout pré-module de cycles $M$ et tout schéma intègre $X$ de point générique $\eta$, le groupe $A^{0}(X ; M)=\cap_{x \in X^{(1)}} \operatorname{ker}\left(\partial_{x}^{\eta}\right)$ est bien défini. Nous aurons besoin de la caractérisation suivante, due à M. Rost (cf. loc. cit. 2.3), des modules de cycles sur un corps parfait:

ThÉoRÈme 4.2.4 [Rost]. Soit $M$ un pré-module de cycles. Alors, $M$ est un module de cycles si et seulement si les conditions suivantes sont vérifiées:

(FDL) Pour tout corps de fonctions $L$ et pour tout $\rho \in L(t)$, l'ensemble $\left\{v\right.$ valuation géométrique sur $\left.L(t) / L \mid \partial_{v}(\rho) \neq 0\right\}$ est fini.

(WR) Pour tout corps de fonctions $L$, notant $\partial_{\infty}$ le résidu associé à la valuation à l'infini de $L(t)$,

$$
\partial_{\infty}\left(A^{0}\left(\mathbb{A}_{L}^{1} ; M\right)\right)=0 .
$$

La référence pour la démonstration de ce théorème est [Ros96, (2.3)].

4.2.5. Pour vérifier les axiomes d'un module de cycles, nous n'utiliserons pas directement le théorème précédent mais nous passerons par la propriété suivante:

Soit $M$ un pré-module de cycles qui satisfait la propriété (FDL). On introduit la propriété suivante:

(H) Pour tout corps de fonctions $L$, notant $\varphi: L \rightarrow L(t)$ l'inclusion canonique, la suite

$$
0 \rightarrow M(L) \stackrel{\varphi_{*}}{\longrightarrow} M(L(t)) \stackrel{d^{0}}{\stackrel{A_{L}^{1}, M}{\longrightarrow}} \bigoplus_{x \in\left(A_{L}^{1}\right)^{(1)}} M(\kappa(x)) \rightarrow 0
$$

est un complexe exact.

Remarquons que la propriété (FDL) signifie exactement que le morphisme $d_{\mathrm{A}_{L}^{1}, M}^{0}$ est bien défini. De plus, (WR) est immédiatement conséquence de $\mathbf{( H )}$ d'après la formule R3c. Donc un pré-module de cycles vérifiant (FDL) et $(\mathbf{H})$ est un module de cycles.

\section{Morphismes et relations}

\section{1 - Le foncteur canonique $M^{(0)}$}

De manière frappante, il apparaît que la catégorie $\tilde{\mathscr{E}}_{k}$ permet de décrire les morphismes de motifs génériques par générateurs et relations comme le montre le théorème suivant: 
THÉORÈmE 5.1.1. Il existe un foncteur canonique

$$
M^{(0)}:\left(\tilde{\mathscr{E}}_{k}\right)^{o p} \rightarrow D M_{g m}^{(0)}(k)
$$

qui à un objet $(E, n)$ de $\tilde{\mathscr{E}}_{k}$ associe le motif générique $M_{g m}(E)\{-n\}$.

PREuve: Il s'agit de définir les morphismes D1 à D4 entrant dans la définition de $\tilde{\mathscr{C}}_{k}$ et de vérifier les relations numérotées $\mathrm{R}^{* *}$. Notons que puisque $Z\{1\}$ est inversible pour le produit tensoriel de $D M_{g m}(k)$, il suffit de définir les morphismes de D1, D2, D3 et D4 pour un twist donné sur la source, le reste des morphismes s'en déduisant par produit tensoriel avec $Z\{1\}$ ou $Z\{-1\}$.

(donnée D1) Si $\varphi: E \rightarrow L$ est un morphisme de corps de fonctions, il lui correspond d'après le premier point de 3.2.6 un unique morphisme $(\varphi):(L) \rightarrow(E)$ qui induit le morphisme $\varphi^{\sharp}: M_{g m}(L) \rightarrow M_{g m}(E)$. La relation R1a est immédiate.

Notons au passage que le même isomorphisme obtenu dans 3.2.6 montre l'existence de modèles respectifs $(X, x)$ et $(Y, y)$ de $E / k$ et $L / k$ et d'un morphisme dominant $f: Y \rightarrow X$ tel que le diagramme suivant commute:

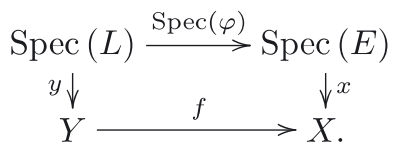

Dans cette situation, on dira que $f:(Y, y) \rightarrow(X, x)$ est un modèle de $\varphi$.

La méthode générale pour construire les morphismes des données D2, D3, D4 est de les construire sur des modèles des corps de fonctions considérés, de vérifier que cette construction se localise aux points génériques et de montrer l'indépendance de la définition par rapport au modèle choisi. La démonstration complète s'étend sur le reste de cette sous-section. La table des matières précise l'endroit où chaque donnée et chaque relation est prouvée.

REMARQUE 5.1.2. On ne perdra pas de vue que le foncteur à construire dans la proposition précédente est contravariant. C'est pour clarifier la situation que l’on a choisi de noter par un symbole $\sharp$ en exposant ou en indice, les morphismes correspondant aux données $D 1$ et $D 2$. 
5.2 - Transferts (D2, R1b, R1c)

\subsection{1 - Situation générique}

LEMme 5.2.1. Soit $f: X \rightarrow Y$ un morphisme fini équidimensionnel.

Alors pour tout ouvert dense $U$ de $X$, l'ouvert $f^{-1}(Y-f(X-U))$ est dense et inclus dans $U$.

Preuve: L'ensemble $Z=X-U$ est un fermé de codimension supérieure à 1 dans $X$, puisque $U$ est dense. En particulier, $f(Z)$ est un fermé de codimension supérieure à 1 dans $Y$, puisque $f$ est fini. Dès lors, $Y-f(Z)$ est un ouvert de $Y$, dense dans $f(X)$. Son image réciproque est done un ouvert dense de $X$, qui est bien contenue dans $U$.

Corollaire 5.2.2. Soit $f: X \rightarrow Y$ un morphisme fini dominant entre schémas irréductibles. Soit $x$ (resp. y) le point générique de $X$ (resp. Y).

Alors le morphisme canonique de pro-objets $X_{x} \rightarrow X \times_{Y} Y_{y}$ est un isomorphisme.

Preuve: En effet, le lemme précédent montre que l'ensemble des ouverts $X \times_{Y} V$ de $X$, pour $V$ parcourant les ouverts non vides de $Y$ est cofinal dans $\mathcal{V}_{x}(X)$.

5.2.3. Soit $f: X \rightarrow Y$ un morphisme fini dominant de schémas lisses irréductibles. Soit $x$ (resp. $y$ ) le point générique de $X$ (resp. $Y$ ).

Considérons $V$ et $V^{\prime}$ deux ouverts non vides de $Y$, tels que $V^{\prime} \subset V$. D'après 1.1.4, on obtient un diagramme commutatif:

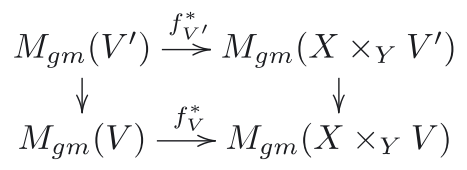

où les morphismes verticaux sont induits par les immersions ouvertes et $f_{V}$ (resp. $f_{V^{\prime}}$ ) désigne le morphisme évident induit par $V$. On peut alors définir le morphisme suivant de pro- $D M_{g m}(k)$

$$
\left(\tau \bar{f}=\lim _{V \in \tilde{V}_{y}(Y)}\left(V \stackrel{f_{V}^{*}}{\longrightarrow} X \times_{Y} V\right)\right): M_{g m}\left(Y_{y}\right) \rightarrow M_{g m}\left(X \times_{Y} Y_{y}\right) .
$$


DÉFInITION 5.2.4. Soit $f: X \rightarrow Y$ un morphisme fini dominant, où $X$ et $Y$ sont des schémas lisses et irréductibles. Soit $x$ (resp. $y$ ) le point générique de $X$ (resp. $Y$ ) ; on note $\bar{f}: X_{x} \rightarrow Y_{y}$ le morphisme induit par $f$.

On définit un morphisme $M_{g m}\left(Y_{y}\right) \stackrel{\bar{f}^{*}}{\longrightarrow} M_{g m}\left(X_{x}\right)$ dans pro- $D M_{g m}(k)$, appelé transposée de $\bar{f}$ en considérant la composée

$$
M_{g m}\left(Y_{y}\right) \stackrel{\tau_{\bar{f}}}{\longrightarrow} M_{g m}\left(X \times_{Y} Y_{y}\right) \stackrel{(1)}{\longrightarrow} M_{g m}\left(X_{x}\right)
$$

où (1) est induit par l'isomorphisme réciproque de 5.2.2.

Lemme 5.2.5. Soient $f: X \rightarrow Y$ et $g: Y \rightarrow Z$ des morphismes finis équidimensionnels avec $X, Y, Z$ des schémas lisses irréductibles, de points génériques respectifs $x, y, z$. Posons $h=g \circ f$. Alors, $\bar{h}^{*}=\bar{f}^{*} \circ \bar{g}^{*}$.

Preuve: Considérons tout d'abord un ouvert non vide $U$ (resp. $V$ ) de $Z$ (resp. $Y$ ) vérifiant $V \subset Y \times_{X} U$. Soit $f_{U}=f \times{ }_{Z} U, g_{U}=g \times_{Z} U$ et $f_{V}=f \times_{Y} V$. D'après la proposition 1.1.4, on obtient le diagramme commutatif suivant:

$$
\begin{array}{r}
M_{g m}(U) \stackrel{g_{U}^{*}}{\longrightarrow} M_{g m}\left(Y \times_{Z} U\right) \stackrel{f_{U}^{*}}{\longrightarrow} M_{g m}\left(X \times_{Z} U\right) \\
M_{g m}(V) \stackrel{f_{V}^{*}}{\longrightarrow} M_{g m}\left(X \times_{Y} V\right) .
\end{array}
$$

D'après la même proposition, ce diagramme est naturel par rapport aux ouverts $U$ et $V$. On peut donc considérer sa limite projective par rapport à $U$ et $V$ pour obtenir le diagramme commutatif suivant:

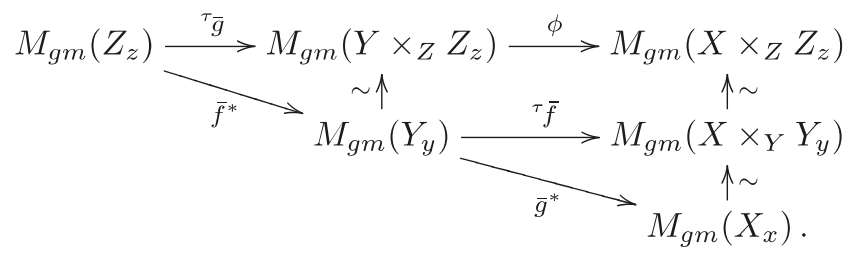

Mais par ailleurs, d'après le lemme 1.1.2, $f_{U}^{*} \circ g_{U}^{*}=\left(g_{U} \circ f_{U}\right)^{*}$. Comme $g_{U} \circ f_{U}=h_{U}$, on en déduit que $\phi \circ{ }^{\tau} \bar{g}={ }^{\tau} \bar{h}$, ce qui permet de conclure.

LEMME 5.2.6. Considérons un carré cartésien dans la catégorie des schémas

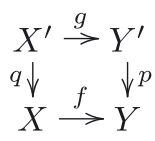


dans lequel $p, q$ sont dominants, $f, f^{\prime}$ sont finis dominants, et $X, Y, Y^{\prime}$ sont lisses et irréductibles.

Pour tout point $z$ dans $X^{(0)}$, le pro-objet $X_{z}^{\prime}$ a pour limite un schéma artinien local, et on a un diagramme commutatif

$$
\begin{gathered}
\left(X_{z}^{\prime}\right)_{\text {red }} \stackrel{\bar{g}_{z}}{\longrightarrow} Y_{y^{\prime}}^{\prime} \\
\bar{q}_{z} \downarrow \\
X_{x} \stackrel{\bar{f}}{\longrightarrow} Y_{y} .
\end{gathered}
$$

Alors, dans pro-DMgm $(k)$,

$$
\bar{f}^{*} \circ \bar{p}_{*}=\sum_{z \in X^{\prime(0)}} \lg \left(\mathcal{O}_{X^{\prime}, z}\right) \cdot \bar{q}_{z *} \circ \bar{g}_{z}^{*} .
$$

Preuve: Comme $k$ est parfait, il existe un ouvert dense $\Omega$ de $X^{\prime}$ tel que $\Omega_{\text {red }}$ est lisse sur $k$.

Soit $V$ un ouvert dense de $Y$ tel que $q^{-1}\left(f^{-1}(V)\right) \subset \Omega$. Posons $U=X \times_{Y} V$ et $V^{\prime}=Y^{\prime} \times_{Y} V$; on a donc un carré cartésien

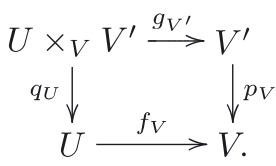

Alors, par définition, $U \times_{V} V^{\prime} \subset \Omega$, et donc $\left(U \times_{V} V^{\prime}\right)_{r e d}$ est lisse sur $k$.

On peut donc appliquer la formule 1.1.4 pour le carré cartésien précédent:

$$
f_{V}^{*} p_{V *}=\sum_{z \in\left(U \times_{V} V^{\prime}\right)^{(0)}} \lg \left(\mathcal{O}_{U \times_{V} V^{\prime}, z}\right) \cdot\left(\left.q_{U}\right|_{z}\right)_{*}\left(\left.g_{V^{\prime}}\right|_{z}\right)^{*}
$$

ce qui permet de conclure puisque $\left(U \times_{V} V^{\prime}\right)^{(0)}=X^{\prime(0)}$ et $\left(U \times_{V} V^{\prime}\right)_{z}=X_{z}^{\prime}$.

\subsection{2 - Modèles et définition}

DÉFINITION 5.2.7. Soient $E$ et $L$ des corps de fonctions et $\varphi: E \rightarrow L$ un morphisme fini. On appelle modèle de $L / E$ tout morphisme $f:(X, x) \rightarrow$ $\rightarrow(Y, y)$ de schémas pointés tel que $(Y, y)$ est un modèle de $E,(X, x)$ un modèle de $L$, et $f$ est un morphisme fini dominant tel que le diagramme suivant commute 


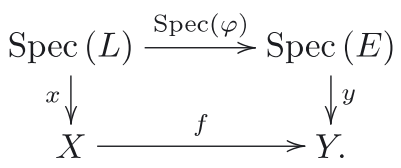

LEMME 5.2.8. Soient $E$ un corps de fonctions et $L / E$ une extension finie. Alors il existe un modèle de $L / E$.

Preuve: Soit $Y_{0}$ un modèle de $E$ et $\tilde{Y}_{0}$ le normalisé de $Y_{0}$ dans $L / E$. Alors $\tilde{Y}_{0}$ est de type fini sur $k$ et $\tilde{Y}_{0}$ est muni d'un $L$-point qui induit un isomorphisme $\kappa\left(\tilde{Y}_{0}\right) \rightarrow L$. On a un morphisme fini canonique $\tilde{Y}_{0} \stackrel{f}{\longrightarrow} Y_{0}$, compatible avec les points canoniques de ces schémas. Enfin, $\tilde{Y}_{0}$ est génériquement lisse. Soit $U$ un ouvert lisse non vide de $\tilde{Y}_{0}$. On pose $Y=Y_{0}-f\left(\tilde{Y}_{0}-U\right)$ et $X=f^{-1}(Y)$. Le lemme 5.2.1 montre que $X$ est non vide et donc $\left.f\right|_{X}: X \stackrel{\left.f\right|_{X}}{\longrightarrow} Y$ convient.

DÉfinition 5.2.9. [D2] Soient $E$ un corps de fonctions et $L / E$ une extension finie; on note $\varphi: E \rightarrow L$ le morphisme structural de $L / E$.

Soit $(X, x) \stackrel{f}{\longrightarrow}(Y, y)$ un modèle de $L / E$.

On définit le morphisme $\varphi_{\sharp}$ de type D2 comme la composée

$$
M_{g m}(E) \stackrel{\sim}{\longrightarrow} M_{g m}\left(Y_{y}\right) \stackrel{\bar{f}^{*}}{\longrightarrow} M_{g m}\left(X_{x}\right) \stackrel{\sim}{\longrightarrow} M_{g m}(L),
$$

les deux isomorphismes étant les isomorphismes canoniques attachés aux modèles (cf. 3.2.6).

5.2.10. On montre que cette définition est indépendante du modèle choisi. Pour cela, on utilise le lemme suivant:

Lemme 5.2.11. Soient $E$ un corps de fonctions et $L / E$ une extension finie. Considérons $X \rightarrow Y$ et $X^{\prime} \rightarrow Y^{\prime}$ deux modèles de L/E. Alors il existe un modèle $X^{\prime \prime} \rightarrow Y^{\prime \prime}$ de $L / E$ et un diagramme commutatif

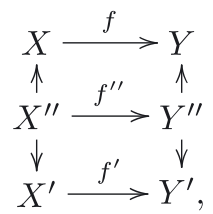

tel que les flèches verticales sont compatibles avec les points génériques des modèles considérés. 
Preuve: On se ramène au cas affine: soit $X=\operatorname{Spec}(B), X^{\prime}=\operatorname{Spec}\left(B^{\prime}\right)$, $Y=\operatorname{Spec}(A), Y^{\prime}=\operatorname{Spec}\left(A^{\prime}\right)$. Puisque $X \rightarrow Y$ et $X^{\prime} \rightarrow Y^{\prime}$ sont des modèles de $L / E$, les isomorphismes canoniques sont compatibles:

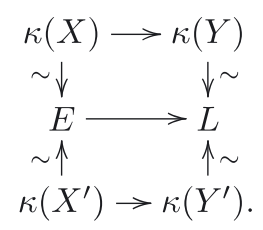

Soient $X=\operatorname{Spec}(A), X^{\prime}=\operatorname{Spec}\left(A^{\prime}\right)$. On peut supposer que $A$ et $A^{\prime}$ sont inclus dans $L$, et $B, B^{\prime}$ inclus dans $E$. Considérons les sous- $k$-algèbres $A^{\prime \prime}=k\left[A \cup A^{\prime}\right] \subset E$ et $B^{\prime \prime}=k\left[B \cup B^{\prime}\right] \subset L$. Tout élément de $B \cup B^{\prime}$ est entier sur $A^{\prime \prime}$, ce qui implique que $B^{\prime \prime} / A^{\prime \prime}$ est intégrale. En particulier, la clôture normale de $A^{\prime \prime}$ dans $L / E$ contient $B^{\prime \prime}$, donc elle contient $B$ et $B^{\prime}$. Notant $X^{\prime \prime}$ son spectre, et $Y^{\prime \prime}=\operatorname{Spec}\left(A^{\prime \prime}\right)$, on obtient un morphisme fini dominant $X^{\prime \prime} \rightarrow Y^{\prime \prime}$, avec le diagramme attendu dans l'énoncé, les morphismes dominants étant donnés par les inclusions canoniques.

Il suffit maintenant de considérer des ouverts de lissité de $X^{\prime \prime}$ et $Y^{\prime \prime}$ pour obtenir les modèles désirés.

Donc, d'après ce lemme, ayant fixé deux modèles $f: X \rightarrow Y$ et $f^{\prime}: X^{\prime} \rightarrow Y^{\prime}$ de $L / E$, on peut supposer qu'il existe un carré cartésien:

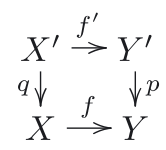

où $p$ (resp. $q$ ) est un morphisme du modèle de $L / k$ (resp. $E / k)$. Or, d'après le lemme 5.2.6 appliqué à ce carré cartésien, on obtient le diagramme commutatif de pro- $D M_{g m}(k)$ suivant

$$
\begin{gathered}
M_{g m}\left(Y_{y^{\prime}}^{\prime}\right) \stackrel{\left(\bar{f}^{\prime}\right)^{*}}{\longrightarrow} M_{g m}\left(X_{x^{\prime}}^{\prime}\right) \\
\bar{p}_{*} \downarrow \\
M_{g m}\left(Y_{y}\right) \underset{\bar{f}^{*}}{\longrightarrow} M_{g m}\left(X_{x}\right) .
\end{gathered}
$$

Ceci permet de conclure puisque $p$ et $q$ sont compatibles aux isomorphismes canoniques des modèles choisis.

Dès lors, la formule R1b est un corollaire du lemme 5.2.5 et la formule $\mathrm{R} 1 \mathrm{c}$ résulte du lemme 5.2.6. 
5.3 - K-théorie (D3, R2a, R2b, R2c)

5.3.1 - Cup-produit générique

Soit $E$ un corps de fonctions. Le morphisme diagonal $\operatorname{Spec}(E) \rightarrow$ $\rightarrow \operatorname{Spec}(E) \times_{k} \operatorname{Spec}(E)$ induit un morphisme dans pro- $D M_{g m}(k)$

$$
\Delta_{E}: M_{g m}(E) \rightarrow M_{g m}(E) \otimes M_{g m}(E) .
$$

Comme dans la définition 1.2.1 (voir aussi la remarque 1.2.3), on en déduit un cup-produit externe sur les morphismes de source $M_{g m}(E)$ :

DÉfinition 5.3.1. Soient $E, L$ et $K$ des corps de fonctions.

Soient $\alpha: M_{g m}(E) \rightarrow M_{g m}(K)\{p\}$ et $\beta: M_{g m}(E) \rightarrow M_{g m}(L)\{q\}$ des morphismes dans $D M_{g m}^{(0)}(k)$.

On définit le cup-produit de $\alpha$ par $\beta$ au-dessus $E$ comme le morphisme

$\alpha \bigotimes_{E} \beta: M_{g m}(E) \stackrel{A_{E}}{\longrightarrow} M_{g m}(E) \otimes M_{g m}(E) \stackrel{\alpha \otimes \beta}{\longrightarrow} M_{g m}(K) \otimes M_{g m}(L)\{p+q\}$

Puisque la permutation des facteurs sur $Z\{1\} \otimes Z\{1\}$ est égale à (-1).Id (cf. [Voe02], 4.8), on en déduit que $\alpha \bigotimes_{E} \beta=-\beta \bigotimes_{E} \alpha$.

Proposition 5.3.2. Soient $E$ et $L$ des corps de fonctions, et $\varphi: E \rightarrow L$ un morphisme fini.

Alors, on obtient les égalités entre morphismes de pro-DM $M_{g m}(k)$ suivantes:

$$
\begin{aligned}
& \left(\varphi^{\sharp} \bigotimes_{L} 1_{L}\right) \circ \varphi_{\sharp}=1_{E} \bigotimes_{E}\left(\varphi_{\sharp}\right), \\
& \left(1_{L} \bigotimes_{L} \varphi^{\sharp}\right) \circ \varphi_{\sharp}=\varphi_{\sharp} \bigotimes_{E} 1_{E} .
\end{aligned}
$$

Preuve: Soit $f:(X, x) \rightarrow(Y, y)$ un modèle de $\varphi$. Soit $V$ un ouvert dense de $Y$, et $U=X \times_{Y} V$. D'après la proposition 1.2.4 appliquée au morphisme fini $f_{V}: U \rightarrow V$ induit par $V$, on obtient:

$$
\begin{gathered}
\left(f_{V *} \bigotimes_{U} 1_{M(U)}\right) \circ f_{V}^{*}=1_{M(V)} \bigotimes_{V} f_{V}^{*}, \\
\left(1_{M(U)} \bigotimes_{U} f_{V *}\right) \circ f_{V}^{*}=f_{V}^{*} \bigotimes_{V} 1_{M(V .)}
\end{gathered}
$$

On en déduit les formules attendues en considérant la limite projective de ces égalités suivant les ouverts denses $V$ de $Y$, en utilisant l'isomorphisme du corollaire 5.2.2 et les isomorphismes canoniques attachés aux modèles $(X, x)$ et $(Y, y)$. 
5.3.2 - Cohomologie motivique

On a déjà vu dans la section 2.6.2 que l'on dispose d'un triangle distingué canonique

$$
\mathrm{Z} \rightarrow M_{g m}\left(\mathrm{G}_{m}\right) \stackrel{\rho}{\longrightarrow} \mathrm{Z}\{1\} \stackrel{+1}{\longrightarrow} .
$$

Par définition du produit tensoriel de $D M_{g m}(k)$, on en déduit donc un triangle distingué dans $D M_{g m}(k)$

$$
\bigoplus_{i=1}^{n} M_{g m}\left(\mathrm{G}_{m}^{n-1}\right) \rightarrow M_{g m}\left(\mathrm{G}_{m}^{n}\right) \stackrel{\rho_{n}}{\longrightarrow} Z\{n\} \stackrel{+1}{\longrightarrow}
$$

où le premier morphisme est la somme des morphismes induits par les $n$ immersions fermées consistant à fixer une des coordonnées à 1 .

Considérons $x=\left(x_{1}, \ldots, x_{n}\right)$ un élément de $\left(E^{\times}\right)^{n}$, où $n \in \mathbb{N}$. Il correspond à un unique morphisme $\operatorname{Spec}(E) \rightarrow \mathrm{G}_{m}^{n}$ que l'on peut voir aussi comme un morphisme de pro-objets $(E) \rightarrow \mathrm{G}_{m}^{n}$, encore noté $x$. On en déduit un morphisme dans pro-DM $M_{g m}(k), \alpha_{x}: M_{g m}(E) \stackrel{x_{*}}{\longrightarrow} M_{g m}\left(\mathrm{G}_{m}^{n}\right) \stackrel{\rho_{n}}{\longrightarrow} Z\{n\}$.

On obtient ainsi une application

$$
\left(E^{\times}\right)^{\otimes, n} \rightarrow \operatorname{Hom}_{D M_{g m}^{(0)}(k)}\left(M_{g m}(E), Z\{n\}\right), x \mapsto \alpha_{x}
$$

puisqu'on vérifie que $\alpha_{x}$ dépend multi-linéairement de $x$. Pour $x \in\left(E^{\times}\right)^{\otimes, n}$ et $y \in\left(E^{\times}\right)^{\otimes, m}$, par définition du cup-produit, $\alpha_{x \otimes y}=\alpha_{x} \bigotimes_{E} \alpha_{y}$. On a donc défini un morphisme d'algèbre

$$
\mathcal{S}\left(E^{\times}\right) \rightarrow \operatorname{Hom}_{D M_{g m}^{(0)}(k)}\left(M_{g m}(E), Z\{*\}\right)
$$

Le théorème suivant se déduit aisément du théorème 3.4 de [SV00]:

THÉORÈME 5.3.3 [Suslin-Voevodsky]. Le morphisme d'algèbre définit ci-dessus se factorise et induit un isomorphisme

$$
K_{*}^{\mathrm{M}}(E) \stackrel{\alpha}{\longrightarrow} \operatorname{Hom}_{D M_{g m}^{(0)}(k)}\left(M_{g m}(E), Z\{*\}\right) .
$$

Remarque 5.3.4. L'analogue du lemme 3.4.4 de loc.cit. tel qu'il est décrit dans la démonstration ci-dessus affirme que pour tout corps de fonctions $E$ et toute extension finie $\varphi: E \rightarrow L$, le morphisme induit par $\varphi_{\sharp}$ sur $\underline{H}^{0}(Z\{*\})$ coïncide à travers l'isomorphisme ci-dessus avec le morphisme norme en K-théorie de Milnor. 
DÉFINITION 5.3.5. [D3] Soient $E$ un corps de fonctions, $n$ un entier naturel et $x \in K_{n}^{\mathrm{M}}(E)$. On considère $\alpha_{x}: M_{g m}(E) \rightarrow Z\{n\}$ le morphisme associé à $x$ par le théorème précédent et on pose: $\gamma_{x}=\alpha_{x} \bigotimes_{E} 1_{E}$.

Lemme 5.3.6. Soient $E, L$ des corps de fonctions, et $\varphi: E \rightarrow L$ un morphisme. Alors, pour tout $x \in K_{*}^{\mathrm{M}}(E)$ et tout $y \in K_{*}^{\mathrm{M}}(L)$ :

R2a: $\gamma_{x} \circ \varphi^{\sharp}=\varphi^{\sharp} \circ \gamma_{\left(\varphi_{*} x\right)}$.

R2b: Si $\varphi$ est fini, $\gamma_{\left(\varphi_{*} x\right)} \circ \varphi_{\sharp}=\varphi_{\sharp} \circ \gamma_{x}$.

R2c: Si $\varphi$ est fini, $\varphi^{\sharp} \circ \gamma_{y} \circ \varphi_{\sharp}=\gamma_{\varphi^{*}}$.

Dans ces formules, $\varphi_{*} x$ désigne l'image directe de $x$ dans $K_{*}^{\mathrm{M}}(L)$ et $\varphi^{*} y$ la norme de $y$ dans $K_{*}^{\mathrm{M}}(E)$.

Preuve: Notons que par définition, $\alpha_{\varphi_{x} x}=\alpha_{x} \circ \varphi^{\sharp}$. Par ailleurs, d'après la remarque 5.3.4, on a aussi $\alpha_{\varphi^{*} y}=\alpha_{y} \circ \varphi_{\sharp}$. Dès lors, les trois propriétés énoncées résultent des propriétés analogues (2), (3) et (4) de la proposition 1.2.4.

5.4 - Résidu (D4, R3a, b, c, d, e)

\subsection{1 - Modèles et définitions}

Soient $E$ un corps de fonctions, et $v$ une valuation géométrique de $E$. Rappelons que par définition l'anneau de $v$, que l'on note $\mathcal{O}_{v}$ est une $k$ algèbre essentiellement lisse.

DÉFINITION 5.4.1. Soient $E$ un corps de fonctions, et $v$ une valuation géométrique de $E$. Considérons $\mathcal{O}_{v}$ l'anneau de valuation de $v$, et $(X, t)$ un modèle de $\mathcal{O}_{v}$. On appelle point spécial de $(X, t)$ l'image du point fermé de $\operatorname{Spec}\left(\mathcal{O}_{v}\right)$ par $t$, noté $s$. On dit que $(X, t)$ est un modèle strict de $\mathcal{O}_{v}$ si l'adhérence réduite de $\{s\}$ dans $X$ est un schéma lisse.

REMARque 5.4.2. Par la suite, nous confondrons simplement le morphisme $t$ et le point $s$ du modèle $X$ dans la situation de la définition précédente.

LEMme 5.4.3. Tout anneau de valuation discrète $\mathcal{O}_{v}$ essentiellement de type fini sur $k$ admet un modèle strict. 
Preuve: Considérons un modèle $(X, s)$ de l'anneau local-lisse $\mathcal{O}_{v}$. Soit $Z$ l'adhérence réduite de $\{s\}$ dans $X$. Alors, $Z$ est un schéma intègre génériquement lisse sur $k$ puisque $k$ est parfait. Il existe donc un ouvert $U$ de $X$ contenant $s$ tel que $Z \cap U$ est lisse sur $k$; $(U, s)$ convient.

5.4.4. Reprenons la situation d'un corps de fonctions $E$ et d'une valuation géométrique de $E$ d'anneau $\mathcal{O}_{v}$. Soient $(X, s)$ un modèle strict de $\mathcal{O}_{v}$ et $Z$ l'adhérence réduite de $s$ dans $X$. On peut considérer la paire fermée $(X, Z)$ lisse de codimension 1. On lui a associé dans 2.3.1 le triangle de Gysin

$$
M_{g m}(Z)\{1\} \stackrel{\partial_{X, Z}}{\longrightarrow} M_{g m}(X-Z) \stackrel{j_{*}}{\longrightarrow} M_{g m}(X) \stackrel{+1}{\longrightarrow} .
$$

Par ailleurs, d'après le théorème 2.4.4, ce triangle est naturel par rapport aux inclusions entre les ouverts de $X$. On en déduit donc en considérant le système cofiltrant des voisinages ouverts de $s$ dans $X$ le pro-triangle distingué

$$
M_{g m}\left(Z_{s}\right)\{1\} \stackrel{\partial_{X_{s}, Z_{s}}}{\longrightarrow} M_{g m}\left(X_{s}-Z_{s}\right) \stackrel{j_{s *}}{\longrightarrow} M_{g m}\left(X_{s}\right) \stackrel{+1}{\longrightarrow} .
$$

Puisque $(X, s)$ est un modèle de $\mathcal{O}_{v}$, le morphisme $s: \operatorname{Spec}\left(\mathcal{O}_{v}\right) \rightarrow X$ induit un isomorphisme de pro-objet $\left(\mathcal{O}_{v}\right) \rightarrow X_{s}$; on en déduit donc un pro-triangle distingué isomorphe au précédent

$$
M_{g m}(\kappa(v))\{1\} \stackrel{\partial_{x_{s}, Z_{s}}^{\prime}}{\longrightarrow} M_{g m}(E) \stackrel{i^{\sharp}}{\longrightarrow} M_{g m}\left(\mathcal{O}_{v}\right) \stackrel{+1}{\longrightarrow}
$$

où $E$ (resp. $\kappa(v))$ est le corps des fractions (resp. résiduel) de $v, i: \mathcal{O}_{v} \rightarrow E$ est le morphisme d'inclusion canonique, et $i^{\sharp}$ se déduit de $i$ comme dans la construction du morphisme D1.

Avant d'arriver à la définition finale, nous utilisons le lemme suivant:

Lemme 5.4.5. Soient $E$ un corps de fonctions et $v$ une valuation géométrique de $E$ d'anneau $\mathcal{O}_{v}$ et de corps résiduel $\kappa(v)$.

Alors, adoptant les notations qui précèdent, si $(X, s)$ et $(Y, t)$ sont deux modèles stricts de $\mathcal{O}_{v}$, notant $Z$ (resp. T) l'adhérence réduite de $\{s\}$ dans $X$ (resp. $\{t\}$ dans $Y$ ),

$$
\partial_{X_{s}, Z_{s}}^{\prime}=\partial_{Y_{t}, T_{t}}^{\prime} .
$$

Preuve: On commence par dominer les modèles en jeu:

On peut supposer tout d'abord que $X$ et $Y$ sont affines quitte à les remplacer par des voisinages ouverts du point spécial. On note $A$ et $B$ les 
anneaux respectifs de $X$ et $Y$. On identifie $A_{s}$ et $B_{t}$ à $\mathcal{O}_{v}$. Donc on a $A \subset \mathcal{O}_{v}$ et $B \subset \mathcal{O}_{v}$. Alors, si l'on choisit $\pi$ une uniformisante de $\mathcal{O}_{v}, s$ (resp. $t$ ) correspond à l'idéal engendré par $\pi$ dans $A$ (resp. $B$ ), et $\pi \in A \cap B$; d'après l'identification ci-dessus, $A_{\pi}=\mathcal{O}_{v}=B_{\pi}$.

Soit $C=k[A \cup B]$, vue comme sous-k-algèbre de $\mathcal{O}_{v}$. Alors $C_{\pi}=\mathcal{O}_{v}$; donc $\operatorname{Spec}(C)$ est formellement lisse sur $k$ au point correspondant à l'idéal $\pi C$. Comme $C / k$ est de type fini, on en déduit un élément $h \in C$ non divisible par $\pi$ tel que $C_{h}$ est lisse sur $k$.

Il ne reste plus qu'à poser $\Omega=\operatorname{Spec}\left(C_{h}\right)$. On a donc par définition des morphismes dominants $\Omega \stackrel{f}{\longrightarrow} X$ et $\Omega \stackrel{g}{\longrightarrow} Y$. L'élément $\pi$ engendre un idéal de $k[C]_{h}$ (non nul) qui correspond à un point $w$ de $\Omega$ de codimension 1 . Enfin, on a encore $\Omega_{w}=X_{s}=Y_{t}$ (modulo notre identification).

Ainsi, on peut donc supposer qu'il existe un morphisme $(Y, t) \stackrel{f}{\longrightarrow}(X, s)$ de modèles de $\mathcal{O}_{v}$. Quitte de nouveau à remplacer $X$ et $Y$ par des voisinages du point spécial, on peut supposer que $f^{-1}(Z)=T$. Autrement dit, $f$ définit un morphisme cartésien de paires fermées lisses $(f, g):(Y, T) \rightarrow(X, Z)$. Notons $h: Y-T \rightarrow X-Z$ le morphisme induit par $f$. Soit $V / U$ un voisinage ouvert de $t / s$ dans $Y / X$. D'après le théorème 2.4.4 appliqué au morphisme cartésien $\left(\left.f\right|_{V} ^{U},\left.g\right|_{V} ^{U}\right)$, on en déduit un morphisme de triangles

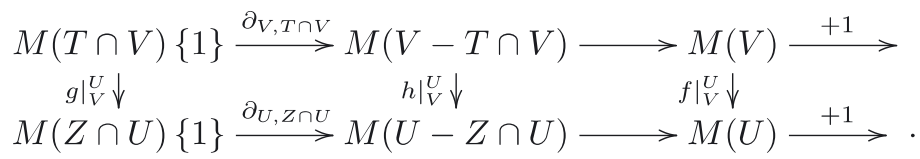

Par passage à la limite, on en déduit donc un diagramme commutatif de proobjets

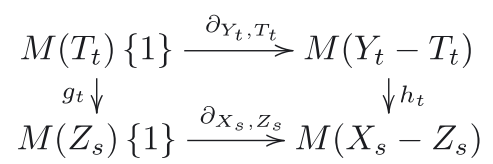

qui permet de conclure puisque le morphisme $g:(T, t) \rightarrow(Z, s)$ et le morphisme $h:(Y-T, t) \rightarrow(X-Z, s)$ sont des morphismes entre modèles de $\kappa(v)$ et $E$.

DÉFINITION 5.4.6. [D4] Soit $E$ un corps de fonctions, et $v$ une valuation géométrique de $E$.

On définit le morphisme résidu associé à $v$, noté $\partial_{v}$, en considérant un modèle strict $(X, s)$ de l'anneau de valuation de $v$ et en posant avec les notations précédentes $\partial_{v}=\partial_{\left(X_{s}, Z_{s}\right)}^{\prime}$. 
On dispose donc d'un pro-triangle distingué

$$
M_{g m}(\kappa(v))\{1\} \stackrel{\partial_{v}}{\longrightarrow} M_{g m}(E) \stackrel{i^{\sharp}}{\longrightarrow} M_{g m}\left(\mathcal{O}_{v}\right) \stackrel{+1}{\longrightarrow} .
$$

\subsection{2 - Relations}

On en déduit tout d'abord les relations suivantes qui concernent le résidu et les données $D 1$ et $D 2$ :

Lemme 5.4.7. Soient $E$ un corps de fonctions et $v$ une valuation géométrique de $E$.

R3a: Soient $L$ un corps de fonctions et $\varphi: L \rightarrow E$ un morphisme tel que $v$ est nulle sur $E$. Alors, $\varphi^{\sharp} \circ \partial_{v}=0$.

R3b: Soient $L$ un corps de fonctions et $\varphi: L \rightarrow E$ un morphisme tel que $\left.v\right|_{L^{\times}}=e . w$ où $w$ est une valuation sur $L$, et $e>0$ un entier. Alors,

$$
\varphi^{\sharp} \circ \partial_{v}=e \cdot\left(\partial_{w}\{1\}\right) \circ \bar{\varphi}^{\sharp}
$$

où $\bar{\varphi}: \kappa(w) \rightarrow \kappa(v)$ est le morphisme induit.

R3c: Soient $L$ un corps de fonctions et $\varphi: E \rightarrow L$ un morphisme fini. On note $\left(w_{i}\right)_{i=1, \ldots, n}$ les valuations sur $L$ qui prolongent $v$ (avec un indice de ramification éventuel), et $\bar{\varphi}_{i}: \kappa\left(w_{i}\right) \rightarrow \kappa(v)$ les morphismes induits sur les corps résiduels. Alors,

$$
\varphi_{\sharp} \circ \partial_{v}=\sum_{i=1}^{n} \partial_{w_{i}} \circ\left(\left(\bar{\varphi}_{i}\right)_{\sharp}\{1\}\right) .
$$

Preuve: Compte tenu du triangle distingué $(\mathrm{E})$, la première relation est évidente puisque $\varphi$ se factorise par le morphisme $i: \mathcal{O}_{v} \rightarrow E$.

Pour la deuxième relation, on considère $(X, s)$ et $(Y, t)$ des modèles respectifs de $(L, w)$ et $(E, v)$; on note $Z$ (resp. $T$ ) l'adhérence réduite de $s$ dans $X$ (resp. $t$ dans $Y$ ). Quitte à réduire $X$ et $Y$, le morphisme $\varphi$ correspond à un morphisme $f: Y \rightarrow X$ tel que $\left(Y \times_{X} Z\right)_{\text {red }}=T$; il induit un morphisme de paires fermées $f:(Y, T) \rightarrow(X, Z)$. Quitte à réduire $X$ et $Y$, ce morphisme est exact d'ordre $e$. Le théorème 2.4 .10 permet donc de conclure.

Pour la dernière relation, on procède de même en imposant de plus que $f$ est fini (grâce au lemme 5.2.1). C'est alors la proposition 2.5.2 qui permet de conclure. 
On obtient finalement les deux dernières relations:

LEMME 5.4.8. Soient $E$ un corps de fonctions et $v$ une valuation géométrique de $E$.

R3d: Soient $\pi$ une uniformisante de $v, L$ un corps de fonctions, et $\varphi: L \rightarrow E$ un morphisme tel que $v$ est nul sur L. Alors, si $\bar{\varphi}: L \rightarrow \kappa(v)$ désigne le morphisme induit par $\varphi$,

$$
\left(\varphi^{\sharp}\{1\}\right) \circ \gamma_{\{-\pi\}} \circ \partial_{v}=\bar{\varphi}^{\sharp}\{1\} .
$$

R3e: Pour tout $u \in E$ tel que $v(u)=0$, notant $\bar{u}$ la classe de $u$ dans le corps résiduel de $v$,

$$
\gamma_{\{u\}} \circ \partial_{v}=-\left(\partial_{v} \circ \gamma_{\{\bar{u}\}}\right)\{1\}
$$

où $\bar{\varphi}: \kappa(w) \rightarrow \kappa(v)$ est le morphisme induit.

Preuve: On fixe $(X, s)$ un modèle strict de $(E, v)$, de point générique $x$, et on note $Z$ l'adhérence réduite de $s$ dans $X$.

Pour la première relation, on considère un modèle $(Y, y)$ de $L$. Quitte à réduire $X$ au voisinage de $s$ et $Y$ au voisinage de $y$, il existe un morphisme $f: X \rightarrow Y$ qui est un modèle de $\varphi$. Alors, le morphisme $g=\left.f\right|_{Z}$ est un modèle de $\bar{\varphi}$. Quitte à réduire à nouveau $X$ au voisinage de $s,(-\pi)$ correspond à une fonction régulière $\pi: X \rightarrow A_{k}^{1}$ et l'on peut supposer qu'elle paramétrise $Z$ dans $X$. Dès lors, il ne reste plus qu'à appliquer la proposition 2.6.5 à la paire fermée $(X, Z)$ et à la fonction $\pi$. En composant la relation que donne cette proposition avec $f$ à gauche, et en considérant la limite projective formelle de ces relations suivant les voisinages de $s$ dans $X$, on obtient la relation attendue.

Pour la deuxième relation, on suppose cette fois que $u$ se relève en une fonction régulière $u: X \rightarrow \mathbb{G}_{m}$. On peut alors considérer le diagramme commutatif suivant:

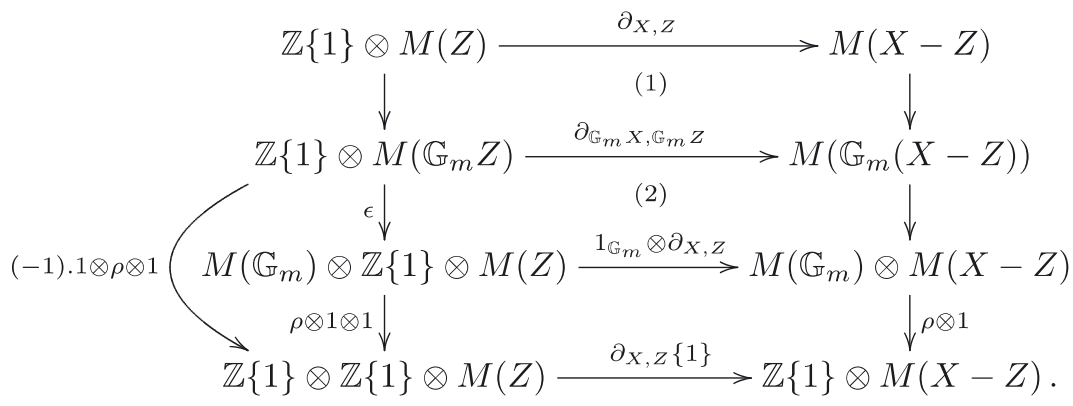


La partie (1) est commutative d'après le théorème 2.4.4. Pour la portion (2), on a noté $\varepsilon$ l'isomorphisme de permutation des facteurs, et on a appliqué la proposition 2.6.1. Le signe résulte du fait que la permutation des facteurs sur $Z\{1\} \otimes Z\{1\}$ est égale à -1 (et du fait que $Z\{1\}$ est facteur direct de $\left.M\left(\mathrm{G}_{m}\right)\right)$. En considérant la limite projective formelle par rapport aux voisinages ouverts de $s$ dans $X$, on obtient la relation attendue.

\section{Structures de module de cycles}

\section{1 - Extensions transcendantes pures}

A l'aide des morphismes de la section précédente, on peut construire le pro-triangle distingué suivant, qui est en quelque sorte une loi de réciprocité faible pour les motifs génériques:

Proposition 6.1.1. Soient $L$ un corps de fonctions et $\varphi: L \rightarrow L(t)$ l'inclusion canonique.

Tout point fermé $x \in \mathrm{A}_{L}^{1}$ correspond à une valuation géométrique sur $L(t)$ notée $v_{x}$. Alors la famille de morphismes

$$
\partial_{v_{x}}: M_{g m}(\kappa(x))\{1\} \rightarrow M_{g m}(L(t))
$$

induit un morphisme bien défini

$$
\partial_{L(t) / L}: \prod_{x \in \mathrm{A}_{L(0)}^{1}} M_{g m}(\kappa(x))\{1\} \rightarrow M_{g m}(L(t)) .
$$

De plus, les morphismes suivants définissent un pro-triangle distingué de $D M_{g m}^{(0)}(k)$ qui est de plus scindé

$$
\prod_{x \in\left(A_{L}^{1}\right)(0)} M_{g m}(\kappa(x))\{1\} \stackrel{\partial_{L(t) / L}}{\longrightarrow} M_{g m}(L(t)) \stackrel{\varphi^{\sharp}}{\longrightarrow} M_{g m}(L) \stackrel{+1}{\longrightarrow} .
$$

Preuve: Soit $(X, \eta)$ un $k$-modèle irréductible de $L$. On considère la catégorie cofiltrante $\mathcal{I}$ ayant

(1) pour objets les paires fermées lisses de codimension 1 qui sont de la forme $\left(\mathbb{A}_{U, Z}^{1}\right)$ telle que $U$ est un ouvert dense de $X, Z$ est intègre et $Z / U$ est fini surjectif,

(2) pour morphismes les morphismes cartésiens de paires fermées qui sont des immersions ouvertes. 
Soit $\left(\mathrm{A}_{U, Z)}^{1} \in \mathcal{I}\right.$. On considère le triangle de Gysin associé à cette paire fermée

$$
M_{g m}(Z\{1\}) \rightarrow M_{g m}\left(\mathbb{A}_{U}^{1}-Z\right) \rightarrow M_{g m}\left(\mathbb{A}_{U}^{1}\right) \stackrel{+1}{\longrightarrow} .
$$

D'après 2.4.4, ce triangle est naturel par rapport aux morphismes de $\mathcal{I}$. Considérant la limite projective formelle de ces triangles distingués, on obtient done un pro-triangle distingué

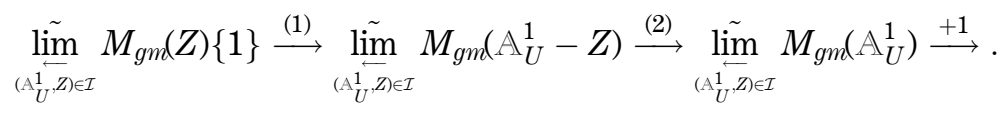

Or, l'ensemble des ouverts de $\mathrm{A}_{X}^{1}$ de la forme $\mathrm{A}_{U}^{1}-Z$ pour $\left(\mathrm{A}_{U}^{1}, Z\right) \in \mathcal{I}$ est cofinal dans l'ensemble des ouverts non vides de $A_{X}^{1}$ (c'est en effet évident pour les ouverts principaux). Ce fait joint à l'invariance par homotopie montre que le morphisme (2) est isomorphe au morphisme $\varphi^{\sharp}: M_{g m}(L(t)) \rightarrow M_{g m}(L)$.

Soit $\left(\mathrm{A}_{U}^{1}, Z\right) \in \mathcal{I}$. On pose $Z_{L}=Z \times_{U} \operatorname{Spec}(L)$. Comme $Z / U$ est fini surjectif, $Z_{L} / L$ est fini surjectif; c'est donc un fermé non vide de $A_{L}^{1}$ de dimension nulle. Il est de plus régulier et irréductible, donc réduit à un point de $\mathrm{A}_{L_{(0)}}^{1}$.

Pour tout $x \in \mathrm{A}_{L_{0}}^{1}$, on considère $\mathcal{I}_{x}$ la sous-catégorie pleine de $\mathcal{I}$ formée des objets $\left(\mathrm{A}_{U}^{1}, Z\right) \in \mathcal{I}$ tels que $Z_{L}=\{x\}$. On remarquera que $\mathcal{I}=\bigsqcup_{x \in \mathrm{A}_{L_{(0)}}^{1}} \mathcal{I}_{x}$.

Or, le morphisme suivant

$$
\underset{\left(\mathcal{A}_{U}^{1}, Z\right) \in \mathcal{I}_{x}}{\sim} \underset{\operatorname{mon}}{\sim}\left(M_{g m}(Z)\{1\} \rightarrow M_{g m}\left(\mathrm{~A}_{U-Z}^{1}\right)\right)
$$

est isomorphe au morphisme $\partial_{v_{x}}: M_{g m}(\kappa(x))\{1\} \rightarrow M_{g m}(L(t))$. En effet, pour tout $\left(\mathrm{A}_{U}^{1}, Z\right) \in \mathcal{I}_{x},\left(\mathrm{~A}_{U}^{1}, Z\right)$ est un $k$-modèle strict de $\left(L(t), v_{x}\right)$, et $\mathcal{I}_{x}$ est une partie cofinale des $k$-modèles stricts de $\left(L(t), v_{x}\right)$.

Enfin, le morphisme composé

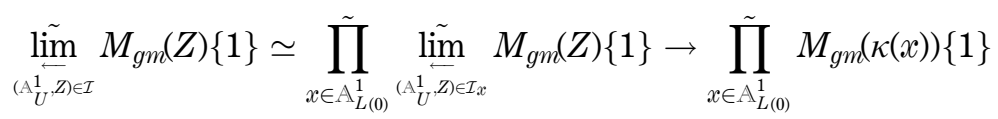

est un isomorphisme. Ceci nous permet d'identifier le morphisme (1) au morphisme $\partial_{L(t) / L}$ de l'énoncé et démontre que ce morphisme est bien défini.

Pour conclure, on remarque que le morphisme $\partial_{L(t) / L}$ admet une rétraction: soit $x \in \mathbb{A}_{L}^{1}$ un point fermé, $\tau_{x}: L(t) \rightarrow \kappa(x)(t)$ et $\varphi_{x}: \kappa(x) \rightarrow$ 
$\rightarrow \kappa(x)(t)$ les inclusions canoniques. Puisque $t-x$ est un élément inversible de $\kappa(x)(t)$, on peut considérer à partir des morphismes de type D1, D2 et D3 le morphisme suivant:

$$
\phi_{x}=\left(\varphi_{x}^{\sharp}\{1\}\right) \circ \gamma_{t-x} \circ\left(\tau_{x}\right)_{\sharp} .
$$

Posons $\phi=\prod_{x \in A_{k_{(0)}}^{1}} \phi_{x}$. Alors, d'après les relations R3b-R3e, on obtient: $\phi \circ \partial_{k(t) / k}=1$.

REMARque 6.1.2.

(1) Compte tenu de l'équivalence de catégorie de 3.4.7, on peut reformuler le résultat de la proposition précédente comme suit: la suite courte suivante de la catégorie abélienne pro- $\mathrm{H} \mathscr{N}_{k}^{\mathrm{tr}}$

$$
0 \rightarrow \prod_{x \in A_{L_{(0)}}^{1}} \underline{\mathrm{H}}^{0}\left(M_{g m}(\kappa(x))\{1\}\right) \stackrel{\underline{\mathrm{H}}^{0} \partial_{L(t) / L}}{\longrightarrow} \underline{\mathrm{H}}^{0} M_{g m}(L(t)) \stackrel{\mathrm{H}^{0} \varphi^{\sharp}}{\longrightarrow} \underline{\mathrm{H}}^{0} M_{g m}(L) \rightarrow 0
$$

est exacte et scindée.

(2) En utilisant l'homologie singulière définie par Suslin et les méthodes de [Voe00a], on peut définir une section du morphisme $\varphi^{\sharp}$ en terme de correspondances finies «à homotopie près». Cette construction peut se substituer à la définition du morphisme $\phi$ dont la formule est tirée de [Ros96], §2, «Step 2», et fournit une deuxième démonstration de la proposition précédente.

On en déduit le calcul suivant du motif générique d'une extension transcendante pure de degré 1 :

Corollaire 6.1.3. Soient $L$ un corps de fonctions et $\varphi: L \rightarrow L(t)$ l'inclusion canonique. Alors le morphisme

$$
\varphi^{\sharp}: M_{g m}(L(t)) \rightarrow M_{g m}(L)
$$

admet une section $\sigma$ telle que le morphisme

$$
M_{g m}(L(t)) \stackrel{\left(\sigma, \prod_{x \in\left(\mathbb{L}_{L}^{1}\right)(0)} \partial_{v_{x}}\right)}{\longrightarrow} M_{g m}(L) \bigoplus \prod_{x \in\left(\mathbb{A}_{L}^{1}\right)_{(0)}} M_{g m}(\kappa(x))\{1\}
$$

est un isomorphisme. 
Remarque 6.1.4. Ce corollaire illustre le fait intuitif que les motifs génériques sont en quelque sorte les motifs les «plus mixtes possibles».

Notons au passage qu'on obtient aussi le résultat suivant:

Corollaire 6.1.5. Soient $E$, $L$ des corps de fonctions et $\varphi: L \rightarrow E$ un morphisme tel que l'extension $E / L$ soit transcendante pure.

Alors, le morphisme $\varphi^{\sharp}: M_{g m}(E) \rightarrow M_{g m}(L)$ admet une section dans $D M_{g m}^{(0)}(k)$.

PREuve: On l'obtient par récurrence à partir du cas où le degré de transcendance est égal à 1 .

6.2 - Théorème principal

6.2.1 - Enoncé général

THÉORÈme 6.2.1. Soit $\varphi: D M_{g m}(k)^{o p} \rightarrow$ Ab un foncteur additif.

Pour tout $(i, r) \in Z^{2}$ et tout corps de fonctions E, on pose:

$$
K_{i}^{\varphi, r}(E)=\lim _{A \in \overrightarrow{\mathcal{M}}_{\overrightarrow{i s}}(E)} \varphi(M(\operatorname{Spec}(A))\{-i\}(-r)) .
$$

Alors, pour tout $r \in Z, K_{*}^{\varphi, r}$ admet une structure canonique de module de cycles.

Preuve: Il suffit de considérer le cas $r=0$.

On commence par prolonger $\varphi$ en un foncteur $\bar{\varphi}:$ pro $-D M_{g m}(k)^{o p} \rightarrow \mathscr{A b}$ en associant à un pro-objet $\left(\mathcal{M}_{i}\right)_{i \in \mathcal{I}}$ le groupe abélien

$$
\lim _{i \in \mathbb{I}^{o p}} \varphi\left(\mathcal{M}_{i}\right) \text {. }
$$

On en déduit un foncteur $\bar{\varphi}: D M_{g m}^{(0)}(k)^{o p} \rightarrow \not b$. Ainsi, par composition avec le foncteur $M^{(0)}$ du théorème 5.1.1, on en déduit un pré-module de cycles, $K_{*}^{\varphi, 0}$ sur $k$.

Mais par ailleurs, puisque $\varphi$ est additif, il en est de même de $\bar{\varphi}$. Dès lors, les propriétés (FDL) et (H) (cf. 4.2.4 et 4.2.5) pour le pré-module de cycles $K_{*}^{\varphi, 0}$ résultent de la proposition 6.1.3.

Exemple 6.2.2. On applique en premier lieu cette proposition dans le cas où le foncteur $\varphi$ est le préfaisceau abélien sur $D M_{g m}(k)$ représenté par 
un motif mixte géométrique $\mathcal{M}$. Dans ce cas, on posera simplement $K_{*}^{\varphi, r}=K_{*}^{\mathcal{M}, r}$.

Notons que pour tout $r \in Z, K_{*}^{\mathcal{M}, r} \simeq K_{*}^{\mathcal{M}(r), 0}$. On remarquera aussi que le module de cycles $K_{*}^{\mathcal{M}, 0}$ dépend fonctoriellement de $\mathcal{M}$.

Proposition 6.2.3. Les isomorphismes de 5.3.3 induisent un isomorphisme de modules de cycles:

$$
K_{*}^{\mathrm{M}} \rightarrow K_{*}^{\mathrm{Z}, 0} .
$$

PREUVE: Il s'agit de vérifier la compatibilité des isomorphismes de 5.3.3 avec les données $\mathrm{D} 1$ à $\mathrm{D} 4$. La compatibilité par rapport à $\mathrm{D} 1$ et $\mathrm{D} 4$ résulte de la définition. La compatibilité par rapport à $\mathrm{D} 2$ résulte de la remarque 5.3.4. Enfin, le fait que le résidu de $K_{*}^{Z, 0}$ coïncide avec le résidu de la Kthéorie de Milnor résulte des formules R3d et R3e.

\subsection{2 - Complexes motiviques}

Soit $\mathcal{C}$ un complexe motivique. Il induit donc un foncteur

$$
\gamma_{\mathcal{C}}: D M_{g m}^{e f f}(k) \rightarrow \mathscr{b b}
$$

tel que pour tout schéma lisse $X$, pour tout $(n, p) \in Z \times \mathbb{N}$,

$$
\gamma_{\mathcal{C}}\left(M_{g m}(X)(p)[n]\right)=\operatorname{Hom}_{D M_{-}^{e f f}(k)}(M(X)(p), \mathcal{C}[-n])
$$

On prolonge ce foncteur en posant, pour tout $(\mathcal{M}, p) \in D M_{g m}(k)$,

$$
\bar{\gamma}_{\mathcal{C}}(\mathcal{M}, p)=\lim _{r \geq \max (-p, 0)} \operatorname{Hom}_{D M_{-}^{\text {eff }}(k)}(\mathcal{M}(p+r), \mathcal{C}(r)) .
$$

Dès lors, par application de la proposition 6.2.1, on peut associer à $\bar{\gamma}_{C}$ une famille de modules de cycles, que l'on note $K_{*}^{\mathcal{C}, r}$. D'après la proposition 3.4.4, pour tout corps de fonctions $E$, pour tout entier $(i, r) \in Z^{2}$ tel que $i+r \leq 0$, on obtient

$$
K_{i}^{\mathcal{C}, r}(E)=\underline{\mathrm{H}}^{-r}(\mathcal{C})_{-(i+r)}(E) .
$$

Notons que le théorème de simplification de Voevodsky (cf. [Voe02], 4.10) nous permet d'exprimer facilement les autres termes de ces modules de cycles: pour tout corps de fonctions $E$ et tout $(i, r) \in Z^{2}$ tel que $i+r \geq 0$, on obtient

$$
K_{i}^{\mathcal{C}, r}(E)=\underline{\mathrm{H}}^{r}(\mathcal{C}\{i+r\})(E) .
$$




\subsection{3 - Réalisations}

La proposition 6.2.1 est faite plus généralement pour s'appliquer à tout foncteur de réalisation triangulé associé à une théorie cohomologique donnée.

Une théorie cohomologique à coefficients dans $\mathscr{b} b$ est pour nous un foncteur contravariant $H^{*}: \mathscr{L}_{k}^{o p} \rightarrow \mathscr{b} b^{N}$ à valeur dans les objets $\mathbb{N}$-gradués de $\mathscr{C} b$. Nous ne précisons pas les axiomes exigés sur cette théorie.

Une réalisation triangulée de $H^{*}$ est un foncteur triangulé

$$
R_{H}: D M_{g m}(k)^{o p} \rightarrow D^{+}(\not b)
$$

tel que pour tout schéma lisse $X$, pour tout $i \in \mathbb{N}, H^{i}(X)=H^{i}\left(R_{H}(X)\right)$. Notons que l'existence de ce foncteur impose déjà un grand nombre de propriétés à la théorie cohomologique $H^{*}$. Notamment, elle devient alors une théorie bigraduée: on pose pour tout couple d'entiers $(n, i) \in \mathbb{N} \times Z$ et pour tout schéma lisse $X: H^{n}(X, i)=H^{n}\left(R_{H}(M(X)(i))\right)$.

La proposition précédente nous permet d'associer à une telle théorie cohomologique une famille de modules de cycles $K_{*}^{H, r}$ telle que pour tout corps de fonctions $E$,

$$
K_{i}^{H, r}(E)=\lim _{A \in \mathcal{M}_{\overrightarrow{l i s}}(E / k)} H^{i}(\operatorname{Spec}(A), r+i)=H^{i}(E, r+i) .
$$

Si $X$ est un schéma lisse connexe, on obtient donc d'après [Ros96] le groupe de Chow des 0-cycles de $X$ à coefficients dans $K_{*}^{H, r}$ :

$$
A^{0}\left(X ; K_{*}^{H, r}\right) \subset \oplus_{i \in Z} H^{i}(\kappa(X), r+i) .
$$

Suivant une terminologie classique, on peut qualifier ces groupes de partie non ramifiée de la théorie cohomologique twistée $H^{n}(., i)$.

EXEMPLE 6.2.4. On appliquera particulièrement cette construction aux foncteurs de réalisation construits par A. Huber dans [Hub00] (voir aussi [Hub04]). Plus précisément, on peut appliquer cette construction à la cohomologie étale continue à coefficients rationnels (cf. [Eke90]) ainsi qu'à la cohomologie absolue de Hodge définie par Beilinson (cf. [Beĭ86] et [Beё87]).

Par ailleurs, dans un travail en préparation, nous montrerons comment construire une réalisation attachée à la cohomologie rigide des schémas de caractéristique $p$. La structure de module de cycles sur la cohomologie rigide non ramifiée est entièrement nouvelle et l'existence d'une action canonique de la K-théorie de Milnor sur la cohomologie rigide non rami- 
fiée est un prolongement naturel des travaux de Kato concernant la cohomologie cristalline.

\section{3 - Filtration par coniveau}

Considérons un foncteur cohomologique

$$
R_{H}: D M_{g m}(k)^{o p} \rightarrow \not b b,
$$

et posons pour tout schéma lisse $X$, et tout $(n, m) \in Z^{2}$,

$$
H^{n}(X, m)=R_{H}\left(M_{g m}(X)(-m)[-n]\right) .
$$

Ci-dessous, nous fixons des notations pour la définition de la filtration par coniveau et la suite spectrale qui s'en déduit pour le foncteur $R_{H}$. Nous rappelons par ailleurs le calcul du terme $E_{2}$ puisqu'il s'insère particulièrement dans la problématique de cet article.

DÉfInITION 6.3.1. Soit $X$ un schéma. On appelle drapeau de $X$ toute suite $\left(Z^{i}\right)_{i \in Z}$ telle que:

(1) Pour tout $i, Z^{i}$ est un sous-schéma fermé de $X$.

(2) Pour tout $i, Z^{i} \supset Z^{i+1}$.

(3) Si $i<0, Z^{i}=X$.

(4) Si $i \geq 0, \operatorname{codim}_{X}\left(Z^{i}\right) \geq i$.

On notera $\mathcal{D}(X)$ l'ensemble des drapeaux de $X$, ordonné par la relation d'inclusion termes à termes.

L'ensemble $\mathcal{D}(X)$ est donc filtrant, et trivialement non vide. Si $X$ est lisse, pour tout drapeau $\left(Z^{i}\right)_{i \in Z}$ de $X$, et pour tout entier $n$, on peut considérer les triangles distingués de $D M_{g m}(k)$ :

$$
M_{g m}\left(X-Z^{n}\right) \rightarrow M_{g m}\left(X-Z^{n+1}\right) \rightarrow M_{g m}\left(X-Z^{n+1}, Z^{n}-Z^{n+1}\right) \stackrel{+1}{\longrightarrow} .
$$

Ces triangles distingués nous permettent de définir un couple exact qui induit une suite spectrale convergente (car $X$ est de dimension de Krull finie) $E_{1}^{p, q}=R_{H}\left(M_{g m}\left(X-Z^{p+1}, Z^{p}-Z^{p+1}\right)[p+q]\right) \Rightarrow R_{H}\left(M_{g m}(X)[p+q]\right)=H^{p+q}(X)$, la filtration sur l'aboutissement de la suite spectrale étant donnée par le noyau des morphismes

$$
H^{p+q}(X) \rightarrow H^{p+q}\left(X-Z^{n}\right)
$$


En considérant la limite inductive suivant le système filtrant des drapeaux de $X$, on en déduit ainsi une suite spectrale convergente

$$
\begin{aligned}
E_{1}^{p, q} & =\lim _{\left(Z^{i}\right)_{i \in Z} \in \mathcal{D}(X)^{p p}} R_{H}\left(M_{g m}\left(X-Z^{p+1}, Z^{p}-Z^{p+1}\right)[p+q]\right) \\
& \Rightarrow H^{p+q}(X) .
\end{aligned}
$$

Pour expliciter ce terme $E_{1}$, on introduit la condition suivante:

DÉFInition 6.3.2. Soit $X$ un schéma lisse et $\left(Z^{i}\right)_{i \in Z}$ un drapeau de $X$.

On dit que $\left(Z_{i}\right)_{i}$ est quasi-lisse si pour tout entier $p$, le schéma $Z^{p}-Z^{p+1}$ est lisse.

Dans le cas d'un drapeau quasi-lisse $\left(Z^{i}\right)_{i}$, l'isomorphisme de pureté 2.2.5 montre que le triangle utilisé ci-dessus est isomorphe au triangle de Gysin

$$
\begin{gathered}
M_{g m}\left(X-Z^{n}\right) \stackrel{j_{*}^{n}}{\longrightarrow} M_{g m}\left(X-Z^{n+1}\right) \stackrel{i_{n}^{*}}{\longrightarrow} M_{g m}\left(Z^{n}-Z^{n+1}\right)(n)[2 n] \\
\stackrel{\partial_{\left(X-Z^{n+1}, Z^{n}-Z^{n+1}\right)}[1]}{\longrightarrow} M_{g m}\left(X-Z^{n}\right)[1]
\end{gathered}
$$

ayant adopté les notations $X-Z^{n} \stackrel{j^{n}}{\longrightarrow} X-Z^{n+1} \stackrel{i_{n}}{\longleftarrow} Z^{n}-Z^{n+1}$ pour les immersions canoniques.

Par ailleurs, si $k$ est parfait, l'ensemble des drapeaux quasi-lisses est cofinal dans l'ensemble des drapeaux de $X$. Utilisant finalement le théorème 2.4.4, on déduit que la suite spectrale par coniveau possède la forme suivante:

$$
E_{1}^{p, q}=\bigoplus_{x \in X^{(p)}} H^{q-p}(\kappa(x),-p) \Rightarrow H^{p+q}(X),
$$

où $X^{(p)}$ désigne l'ensemble des points de codimension $p$ de $X$.

Finalement, avec la notation introduite dans la sous-section précédente, on en déduit:

Proposition 6.3.3. Si H est une théorie cohomologique à valeur dans les groupes abéliens munie d'une réalisation triangulée $R_{H}$, pour tout twist $n \in Z$, la suite spectrale associée à la filtration par coniveau a la forme

$$
E_{1}^{p, q}=C^{p}\left(X ; K_{*}^{H, n-q}, q\right) \Rightarrow H^{p+q}(X, n)^{1} .
$$

( $\left.{ }^{1}\right)$ Nous avons adopté la notation de [Ros96], §5, concernant la bi-graduation naturelle du complexe de cycles à coefficients. 
REMARque 6.3.4. Une question en suspens est l'identification des différentielles de cette suite spectrale avec les différentielles du complexe de cycles à coefficients dans $K_{*}^{H, q}$ définies par Rost. Cette question est résolue dans l'article [Dég05].

\section{4 - Remarques sur la conjecture de Beilinson-Soulé}

Dans cette sous-section, on fixe un anneau de coefficients $A$. On note $D M_{g m}(k, A)$ (resp. $\left.D M_{-}^{e f f}(k, A)\right)$ la catégorie des motifs mixtes géométriques (resp. complexes motiviques) à coefficients dans $A$. La plupart des conjectures introduites ci-dessous sont généralment admises dans le cas $A=\mathrm{Q}$. Le cas $A=Z$ semble plus litigieux, et nous laissons le choix de $A$ au lecteur.

Les résultats que l'on a obtenus jusqu'à présent sont valables avec des motifs mixtes géométriques ou complexes motiviques à coefficients dans $A$, compte tenu du foncteur $D M_{g m}(k) \rightarrow D M_{g m}(k, A)$. On les utilise ici dans ce cadre.

Rappelons la conjecture de Beilinson-Soulé:

(BS) pour tout schéma lisse $X$, pour tout $(n, m) \in \mathbb{N}^{*} \times \mathbb{Z}$,

$$
\operatorname{Ext}_{D M_{g m}(k, A)}^{-n}\left(M_{g m}(X), A(m)\right)=0 .
$$

Le fait que la cohomologie motivique forme un module de cycles ${ }^{2}$ permet de déduire aisément la réduction suivante:

Proposition 6.4.1. Les conditions suivantes sont équivalentes:

(1) La conjecture (BS) est vraie.

(2) Pour tout $r \in Z, K_{*}^{A, r}$ est concentré en degré positif.

(3) Pour tout $r \in Z$, le module de cycles $K_{-1}^{A, r}$ est $n u l$.

PREUve: D'après la proposition 6.3.3 appliquée au foncteur cohomologique de $D M_{g m}(k)$ représenté par $A(m)$, on obtient la suite spectrale

$$
E_{1}^{p, q}=\bigoplus_{x \in X^{(p)}} K_{q-p}^{A, m-q}(\kappa(x)) \Rightarrow \operatorname{Ext}_{D M_{g m}(k)}^{p+q}\left(M_{g m}(X), A(m)\right) .
$$

() C'est plus précisément la proposition 6.1.1 qui est le point clé. 
Le fait que $1 \Rightarrow 2$ est évident. Cette suite spectrale permet de déduire la réciproque.

Pour l'équivalence de 2 et 3 , il nous suffit de remarquer que d'après la propriété (H) des modules de cycles (cf. [Ros96], 2.2), si un module de cycles $M_{*}$ est nul en degré $r$, il est nul en tout degré inférieur à $r$.

La formulation de cette conjecture en termes de complexes motiviques est plus satisfaisante. Pour cela, on a besoin du théorème de simplification de Voevodsky (cf. [Voe02]). En effet, celui-ci implique que pour tout $(n, m) \in Z^{2}$,

$$
\left.\operatorname{Ext}_{D M_{g m}(k, A}^{n}\right)\left(M_{g m}(X), A(m)\right)= \begin{cases}H^{n}(X ; A(m)) & \text { si } m \geq 0 \\ 0 & \text { sinon. }\end{cases}
$$

On déduit d'abord de ce calcul le fait que pour tout $r<0, K_{*}^{A, r}=0$. Par ailleurs, on obtient encore les formulations équivalentes suivantes de la conjecture de Beilinson-Soulé:

CoRollaire 6.4.2. Les conditions suivantes sont équivalentes:

(1) La conjecture (BS) est vraie.

(2) Pour tout $(n, r) \in \mathbb{N}^{*} \times \mathbb{N}, \underline{\mathrm{H}}^{-n}(A(r))=0$.

(3) Pour tout $r \in \mathbb{N}, K_{-1}^{A, r}=0$.

Preuve: Cela résulte simplement du calcul de $K_{*}^{A, r}$ effectué dans 6.2.2 par application du théorème de simplification.

REMARQUE 6.4.3. On peut aussi déduire cette proposition du théorème de simplification et de la proposition 3.4.4. Notons tout d'abord que l'équivalence des deux premières conditions résulte de la suite spectrale d'hypercohomologie. Par ailleurs, rappelons que la démonstration de 3.4.4 implique que le foncteur $\underline{\operatorname{Hom}}_{D M_{-}^{\text {eff }}(k)}(A\{1\},$.$) est exact. Dès lors, si l'on$ pose $A(*)=\bigoplus_{n \in \mathbb{N}} A(n)$, on en déduit

$$
\underline{\mathrm{H}}^{n}(A(*))_{-1}=\underline{\mathrm{H}}^{n}\left(\underline{\operatorname{Hom}}_{D M^{e f f}(k)}(A\{1\}, A(*))\right)=\underline{\mathrm{H}}^{n-1}(A(*)),
$$

la deuxième égalité résultant du théorème de simplification. Ceci entraîne facilement l'équivalence des conditions 2 et 3, grâce à la proposition 3.4.1.

On voit plus généralement que les groupes

$$
\mathrm{H}_{\mathcal{M}}^{n, m}(E ; A)=\operatorname{Hom}_{\text {pro- } D M_{g m}^{e f f}(k)}\left(M_{g m}(E), A(m)[n]\right)
$$


pour $E$ un corps de fonctions variable, s'organisent de la manière suivante:

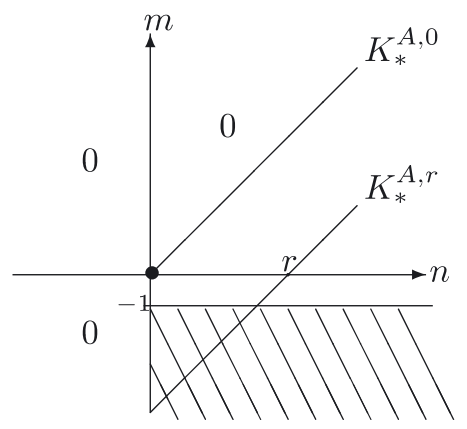

Dans ce graphique les 0 indiquent les parties dont on sait qu'elles sont nulles. Les éléments situés sur la droite désignée $K_{*}^{A, r}$ dans ce graphique s'organisent dans le module de cycles qui à un corps de fonctions $E$ associe $\bigoplus_{i \in Z} \mathrm{H}_{\mathcal{M}}^{i, i+r}(E ; A)$. Rappelons que $K_{*}^{A, 0}=K_{*}^{\mathrm{M}} \otimes A$. Par ailleurs, pour tout couple d'entiers $(r, s)$, le cup-produit en cohomologie motivique induit un «pairing» (cf. [Ros96], 1.2) de modules de cycles $K_{*}^{A, r} \times K_{*}^{A, s} \rightarrow K_{*}^{A, r+s}$. La conjecture de Beilinson-Soulé signifie donc que la partie hachurée du dessin est nulle. On a obtenu dans la démonstration de la première proposition que s'il y a un 0 en coordonnée $(n, m)$, il y a des 0 partout sur la demi-droite $(n+i, m+i)$ pour $i \geq 0$.

B. Kahn nous a signalé que l'on conjecture même l'annulation des groupes situés sur la demi-droite $m=0$ et $n>0$. L'argument que nous avons dégagé montre que cette annulation entraîne la conjecture de Beilinson-Soulé. Par ailleurs, les mêmes méthodes montrent que cette conjecture est encore impliquée par la conjecture de «rigidité» suivante que nous avons apprise de B. Kahn:

ConjeCture DE RIGIDITÉ: Pour tout corps $E$ de corps des constantes $E_{0}$, pour tout entier $n>1$, le morphisme canonique

$$
\mathrm{H}_{\mathcal{M}}^{1, n}\left(E_{0} ; A\right) \rightarrow \mathrm{H}_{\mathcal{M}}^{1, n}(E ; A)
$$

est un isomorphisme.

\section{REFERENCES}

[Beĭ86] A. BEĬLInson, Notes on absolute Hodge cohomology, Applications of algebraic $K$-theory to algebraic geometry and number theory, Part I, II (Boulder, Colo., 1983), Contemp. Math., vol. 55, Amer. Math. Soc., Providence, RI, 1986, pp. 35-68. 
[Beĭ87] A. BeĬLInson, Correction to: "Notes on absolute Hodge cohomology" [Applications of algebraic K-theory to algebraic geometry and number theory, Part I, II (Boulder, Colo., 1983), 35-68, Amer. Math. Soc., Providence, R.I., 1986], K-theory, arithmetic and geometry (Moscow, 1984-1986), Lecture Notes in Math., vol. 1289, Springer, Berlin, 1987, pp. 25-26.

[Beĭ02] A. BEĬLINSON, Remarks on n-motives and correspondences at the generic point, Motives, polylogarithms and Hodge theory, Part I (Irvine, CA, 1998), Int. Press Lect. Ser., vol. 3, Int. Press, Somerville, MA, 2002, pp. 35-46.

[Dég03] F. DÉGLISE, Modules de cycles et motifs mixtes, C. R. Math. Acad. Sci. Paris 336 (2003), no. 1, pp. 41-46.

[Dég04] F. DÉGLISE, Interprétation motivique de la formule d'excès d'intersection, C. R. Math. Acad. Sci. Paris 338 (2004), no. 1, pp. 41-46.

[Dég05] F. DÉGLISE, Around the gysin triangle, Preprint LAGA 2005-30, June 2005.

[Dég06] F. DÉGLISE, Transferts sur les groupes de Chow à coefficients, Math. Z. 252 (2006), no. 2, pp. 315-343.

[Dég07] F. DÉGLISE, Finite correspondances and transfers over a regular base, Algebraic cycles and motives (J. Nagel et P. Chris, eds.), Cambridge university press, april 2007.

[Eke90] T. EkedAHL, On the adic formalism, The Grothendieck Festschrift, Vol. II, Progr. Math., vol. 87, Birkhäuser Boston, Boston, MA, 1990, pp. 197-218.

[Ful98] W. Fulton, Intersection theory, second ed., Springer-Verlag, Berlin, 1998.

[Hub00] A. Huber, Realization of Voevodsky's motives, J. Algebraic Geom. 9 (2000), no. 4, pp. 755-799.

[Hub04] A. Huber, Corrigendum to: "Realization of Voevodsky's motives" [J. Algebraic Geom. 9 (2000), no. 4, 755-799], J. Algebraic Geom. 13 (2004), no. 1, pp. 195-207.

[Kat86] K. Kato, Milnor K-theory and the Chow group of zero cycles, Applications of algebraic $K$-theory to algebraic geometry and number theory, Part I, II (Boulder, Colo., 1983), Contemp. Math., vol. 55, Amer. Math. Soc., Providence, RI, 1986, pp. 241-253.

[MV99] F. Morel et V. Voevodsky, $\mathbf{A}^{1}$-homotopy theory of schemes, Inst. Hautes Études Sci. Publ. Math. (1999), no. 90, pp. 45-143 (2001).

[Ros96] M. Rost, Chow groups with coefficients, Doc. Math. 1 (1996), No. 16, pp. 319-393 (electronic).

[Ser68] J.-P. SERre, Corps locaux, Hermann, Paris, 1968, Deuxième édition, Publications de l'Université de Nancago, No. VIII.

[SV96] A. Suslin - V. Voevodsky, Singular homology of abstract algebraic varieties, Invent. Math. 123 (1996), no. 1, pp. 61-94.

[SV00] A. SusLIN - V. VoEVODSKY, Bloch-Kato conjecture and motivic cohomology with finite coefficients, The arithmetic and geometry of algebraic cycles (Banff, AB, 1998), NATO Sci. Ser. C Math. Phys. Sci., vol. 548, Kluwer Acad. Publ., Dordrecht, 2000, pp. 117-189.

[Voe00a] V. Voevodsky, Cohomological theory of presheaves with transfers, 
Cycles, transfers, and motivic homology theories, Ann. of Math. Stud., vol. 143, Princeton Univ. Press, Princeton, NJ, 2000, pp. 87-137.

[Voe00b] V. Voevodsky, Triangulated categories of motives over a field, Cycles, transfers, and motivic homology theories, Ann. of Math. Stud., vol. 143, Princeton Univ. Press, Princeton, NJ, 2000, pp. 188-238.

[Voe02] V. Voevodsky, Cancellation theorem, K-theory preprint archive 0541, 2002.

[VSF00] V. Voevodsky - A. Suslin - E. M. Friedlander, Cycles, transfers, and motivic homology theories, Annals of Mathematics Studies, vol. 143, Princeton University Press, Princeton, NJ, 2000.

[SGA4] M. Artin, A. Grothendieck - J.-L. Verdier, Théorie des topos et cohomologie étale des schémas, vol. 269,270,305, Springer, 1972-73.

[EGA4] A. GROTHENDIECK - J. DiEUDONNÉ, Eléments de géométrie algébrique IV, vol. 20, 24, 28, 32, Publ. Math. de l'IHES, 1966.

Manoscritto pervenuto in redazione il 19 dicembre 2005 e riveduto il 26 febbraio 2007. 